\title{
Seven Health Physics Calculator Programs for the HP-41CV
}

$+$

August 1984

Paul D. Rittmann

Radiological Protection Department

Health, Safety and Environment Function

Prepared for the U.S. Department of Energy under Contract DE-AC06-77RL01030
RHO-HS-ST- -5 P

DE85 004010

\section{DISCLAIMER}

This report was prepared as an account of work sponsored by an agency of the United States Government. Neither the United States Government nor any agency thereof, nor any of their employees, makes any warranty, express or implied, or assumes any legal liability or responsibility for the accuracy, completeness, or usefulness of any information, apparatus, product, or process disclosed, or represents that its use would not infringe privately owned rights. Reference herein to any specific commercial product, process, or service by trade name, trademark, manufacturer, or otherwise does not necessarily constitute or imply its endorsement, recommendation, or favoring by the United States Government or any agency thereof. The views and opinions of authors expressed herein do not necessarily state or reflect those of the United States Government or any agency thereof.

\section{Rockwell International}

Pockwoll Hentord Oparations Energy 8yotems Oroup PO. Box 800

$\sqrt{\text { Pichlend, Weachingtion } 99352}$

\section{atrote}

"

2


RHO-HS-ST-5 P

DISTRIBUTION

This report has been distributed according to the category "Health and Safety," UC-41, as given in the Standard Distribution for Unclassified

Scientific and Technical Reports, TID-4500 


\section{DISCLAIMER}

This report was prepared as an account of work sponsored by an agency of the United States Government. Neither the United States Government nor any agency Thereof, nor any of their employees, makes any warranty, express or implied, or assumes any legal liability or responsibility for the accuracy, completeness, or usefulness of any information, apparatus, product, or process disclosed, or represents that its use would not infringe privately owned rights. Reference herein to any specific commercial product, process, or service by trade name, trademark, manufacturer, or otherwise does not necessarily constitute or imply its endorsement, recommendation, or favoring by the United States Government or any agency thereof. The views and opinions of authors expressed herein do not necessarily state or reflect those of the United States Government or any agency thereof. 


\section{DISCLAIMER}

Portions of this document may be illegible in electronic image products. Images are produced from the best available original document. 


\section{ABSTRACT}

Several user-oriented programs for the Hewlett-Packard HP-41CV are explained. (This hand-held programmable calculator has alphanumeric display prompting that facilitates data input and continuous memory and simplifies field use.) The first program builds, stores, alters, and ages a list of radionuclides. This program only handles single- and double-decay chains. The second program performs convenient conversions for the six nuclides of concern in plutonium handling. The conversions are between mass, activity, and weight percents of the isotopes. The source can be aged and/or neutron generation rates can be computed. The third program is a timekeeping program that improves the process of manually estimating and tracking personnel exposure during high dose rate tasks by replacing the pencil, paper, and stopwatch method. This program requires a time module. The remaining four programs deal with computations of time-integrated air concentrations at various distances from an airborne release. Building wake effects, source depletion by ground deposition, and sector averaging can a 11 be included in the final printout of the "X/Q - Hanford" and "X/Q - Pasquil1" programs. The shorter versions of these, "H/Q" and "P/Q," compute centerline or sector-averaged values and include a subroutine to facilitate dose estimation by entering dose factors and quantities released. The horizontal and vertical dispersion parameters in the Pasquil1-Gifford programs were modeled with simple, two-parameter functions that agreed very well with the usual textbook graphs. 


\section{CONTENTS}

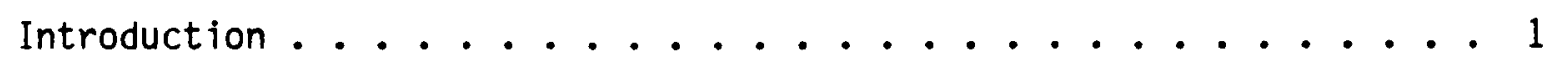

Radionuclide Programs. .............. 1

Radionuclide Decay Programs - "DK" and "DK*" ........ 1

Plutonium Programs - "PU" and "NT". ........... 2

Timekeeping Program. ................ 2

Time-Integrated Air Concentrations ............ 3

Emergency Response - "H/Q". . . . . . . ...... 3

"X/Q - Hanford" .................. 4

Emergency Response - "P/Q". . . . . . . ...... 4

"X/Q - Pasquili"................. 4

Summary and Conclusions. .............. 5

Bibliography ..................... 7

Appendixes:

A. Radionuclide Decay Programs - "DK" and DK*" ........A-1

B. Plutonium Programs - "PU" and "NT"........... B-1

C. Timekeeping Program ............... C-1

D. Emergency Response - "H/Q".............. D-1

E. "X/Q - Hanford" ................. . . . .

F. Emergency Response - "P/Q".............. F-1

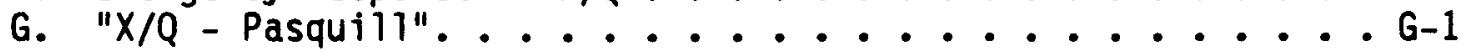




\section{INTRODUCTION}

The Hewlett-Packard HP-41CV is a hand-held calculator with over 120 built-in functions and $2.2 \mathrm{~kb}$ of random access memory. The calculator's memory is retained even when the calculator is turned off, so programs and data need not be read from cards or other storage media whenever the program is to be run. Further, the liquid crystal display can be used for alphanumeric messages of up to 24 characters, which allows the creation of highly user-oriented programs (i.e., programs that guide the user through the necessary data inputs and thereby reduce opportunities for input errors). The widespread use of this calculator, as well as a frequent need for programs of this type to prepare input to the shielding and dose computation computer codes used in radiological analyses by the Radiological Engineering and Effluent Controls group of Rockwe 11 Hanford Operations, Richland, Washington, led to the creation of the radionuclide and timeintegrated air concentration programs. The timekeeping program was developed in response to a request by a facility health physics staff member to improve the accuracy of the manual method used to estimate external dose. Timekeeping is the most reliable, though least accurate, dosimetric tool currently in use.

\section{RADIONUCLIDE PROGRAMS}

Computer codes used by the Radiological Engineering and Effluent Controls group for shielding design, dose rates through existing shielding, and environmental dose calculations all require careful input to obtain meaningful results. All of these codes lack in one or both of the following areas: (1) they cannot age the source initially or (2) they cannot accept plutonium inputs as either masses or activities or by weight percents, given the total mass. To speed the generation of accurate inputs, two HP-4iCV programs were developed. The first is for fission product sources. The second is for. plutonium sources. Accurate computation of daughter ingrowth is assured by use of the HP-41CV function $\left(e^{X}-1\right)$, which is particularly useful at short decay times.

\section{RADIONUCLIDE DECAY PROGRAMS - "DK" AND "DK*"}

This program builds a data base and allows for aging. The userprompting inputs include the alphanumeric identity, half-life, initial quantity, and decay time. The menu for time units allows seconds, minutes, hours, days, or years. Once a data base is entered, the program gives the option of recording it on magnetic cards. If a data base was read from cards, the data entry portion of the program may be skipped. This program runs with or without a printer attached. Input data are displayed, or displayed and printed.

In the HP-41CV, enough memory is free to permit up to 73 radionuclides to be entered. Prior to data entry, the nuclides must be grouped by singleor double-decay kinds. Certain double-decay nuclides may be treated as single-decay types if equilibrium is already established. For example, in 
the $90 \mathrm{Sr}$ and $90 \mathrm{Y}$ sequence, the pair should be treated as a two-step decay chain with a parent half-i ife of $28.6 \mathrm{yr}$, a branching ratio to $90 \mathrm{y}$ of 1.000 , and a daughter half-life of $64 \mathrm{~h}$. However, if the strontium were more than a few weeks old, the two could be treated as single decay nuclides, but both the $90 \mathrm{Sr}$ and $90_{Y}$ would be assigned a haif-1ife of $28.6 \mathrm{yr}$.

Further details on the radionuclide decay program are given in Appendix A. Included in the appendix are detailed program user instructions, a program flowsheet, and an annotated program listing. Program "DK*", which is described at the end of Appendix A, addresses the need to occasionally multiply all the initial activities by a constant factor to scale the source up or down.

\section{PLUTONIUM PROGRAMS - "PU" AND "NT"}

The program "PU" is designed first of all to facilitate weight percent, mass, and activity transformations among the six isotopes of concern in most plutonium handling: $238 \mathrm{Pu}, 239 \mathrm{Pu}, 240 \mathrm{Pu}, 241 \mathrm{Pu}, 242 \mathrm{Pu}$, and $241 \mathrm{Am}$. Second, the program gives total curies of alpha and beta emitters, total mass, and total heat generation rates for the source. Third, the program permits the source to be aged and gives the amounts of the uranium isotopes which build in. Fourth, the program "NT" computes neutron generation rates (neutrons per second) produced from spontaneous fission and alpha-neutron reactions in five materials: oxide, nitrate, fluoride, carbide, or beryllium. The neutron production rates are computed for both the original source and the aged source (if aged). A sample printout is shown in Appendix B.

Detailed user instructions, data tables, a program flowsheet, and annotated program listings are found in Appendix $B$.

\section{TIMEKEEPING PROGRAM}

This program is designed to make the HP-41CV useful in estimating and tracking personnel exposure during high dose rate tasks. The calculator is wrapped in a thin plastic bag to protect it from contamination during field use. A "time module" is a required accessory for this program. Typically, an operator is assigned the task of estimating personnel dose using a stopwatch, pencil, and paper. The HP-41CV replaces these tools.

Program operation is in three parts: initialization, timekeeping, and final printout. During initialization, the program prompts the operator for alphanumeric input of up to five names or identification numbers. Once these are entered, the timekeeping portion begins. The buttons labeled $A$, $B, C, D$, and $E$ are used to update the individual's dose rate. A new dose rate is entered, and the button for that individual is pressed. The calculator then computes the current total dose and displays it along with the current dose rate and button label (A through E). Two functions are available for use during timekeeping. The first is "STATIM," which allows the operator to compute how long it will take an individual to reach a 
specified dose limit at a given dose rate. The second is "DVIEW," which cycles through the names initially entered and displays their current dose rate, button label, and accumulated total dose.

Once the task is complete, zero dose rates are entered to terminate dose accumulation. The dose totals can be read out at this time and recorded manually or a printer can be attached to obtain dose totals for each of the initially entered names as well as the total dose for the task.

Timekeeping is intended to be a fail-safe backup to the usual dosimetry equipment (e.g., self-reading pencils and thermoluminescent dosimeters). Concern that operators might become unable to track doses without a calculator has resulted in limited use of this tool.

Detailed program descriptions are found in Appendix C. Two versions of the program are described there: a version that optimizes speed and a version that minimizes program storage.

\section{TIME-INTEGRATED AIR CONCENTRATIONS}

These programs began as a simple improvement over the existing emergency response calculator program for the HP-67/97 and a dose estimation worksheet. The program "H/Q" replaced the four separate programs and included a subroutine to replace the worksheet. (Two advantages of the HP-41CV version over the HP-67/97 are alphanumeric prompting to minimize data entry errors and an option to view all the input data.) This program was expanded to enable ground deposition corrections and building wake effects. Finally, analogous programs using the Pasquill-Gifford (PG) turbulence classes were developed for the HP-41CV.

\section{EMERGENCY RESPONSE - "H/Q"}

As described in the preceding paragraph, "H/Q" permits computation of either sector-averaged or centerline time-integrated air concentrations and estimation of inhalation or submersion doses from the passing cloud. The program uses the usual gaussian plume model and Hanford dispersion parameters for very stable and moderately stable conditions. Sutton's form has been modified slightly; the vertical dispersion parameter $\left(\sigma_{z}\right)$ is not allowed to exceed $2,000 \mathrm{~m}$.

The dose computation requires entry of curies released and the appropriate dose factor for the isotope. The dose factors used already include a breathing rate and have units of $\left(\mathrm{rem} / \mathrm{Ci} \cdot \mathrm{m}^{3} / \mathrm{s}\right)$.

Detailed user instructions, a flowsheet, and an annotated program listing are given in Appendix $D$. 
"X/Q - HANFORD"

This program was developed to include ground deposition and building wake effects into the normalized, integrated exposure calculation. After considerable testing, a method to speed the numeric integration was developed. To integrate from 0 to $X$, the interval is divided into exponentially increasing segments (similar to a logarithmic scale on graph paper) and Simpson's rule is applied to each. Accuracy of the integral is better than $0.2 \%$ in all cases, and program running time is about $1 \mathrm{~min}$ per distance used.

Detailed user instructions, a sample of output, and an annotated program listing are given in Appendix $E$. The mathematical models used are also described there.

\section{EMERGENCY RESPONSE - "P/Q"}

For comparison with the Hanford model, the program "P/Q" was developed to compute time-integrated exposures for the usual PG classes $A$ through $F$. Because the available formulas poorly represented the vertical dispersion parameter, a new set of parameterizations was developed. These agree with the PG curves to better than 10\% over the entire length of the curves from $0.1 \mathrm{~km}$ to $100 \mathrm{~km}$. Program "P/Q" does for the PG classes what "H/Q" does for the Hanford classes.

Detailed user instructions and an annotated program listing are given in Appendix F.

\section{"X/Q - PASQUILL"}

To complete the time-integrated air concentration program library, ground deposition and building wake effect had to be included in a version of "X/Q" which uses the PG classes. Because the parameterizations of the dispersion parameters lead to a source depletion integral that diverges with zero release height, the program uses a release height of $0.1 \mathrm{~m}$ whenever the input height is less than this.

Detailed user instructions, an annotated program listing, and the mathematical formulas used are included in Appendix G. A sample printout is also presented there. 


\section{SUMMARY AND CONCLUSIONS}

Because the HP-41CV fits into a moderately sized pocket, it is a powerful tool for the workplace. In an office environment, the calculator can be a useful adjunct to larger computer systems that lack the immediate accessibility of a hand-held calculator. The seven programs described in this report, together with numerous other programs currently available, are evidence of the calculator's important function.

Future applications are dependent on future hand-held computing needs. One potential use for the calculator is in Radiation Protection Technologist dose rate logging at standard checkpoints within a facility. The technician would measure various dose rates at a given checkpoint and enter the location and the readings into an HP-4ICV. The calculator would then be interfaced with a desk-top computer to store data and eventually process the data into summary and trend reports. 


\section{BIBLIOGRAPHY}

Hanna, S. R., G. A."Briggs and R. P. Haskar, Jr. (1982), Handbook on Atmospheric Diffusion. National Technical Information Service, Springfield, Virginia.

Kathren, R. L., D. P. Higby, and M. A. McKinney (1984), Computer Applications in Health Physics." Columbia Chapter of the Health Physics Society, Richland, Washington.

Kocher, D. C. (1981), Radioactive Decay Data Tabies.* National Technical Information Service, Springfield, Virginia.

Lederer, C. M. and V. S. Shirley (1978), Table of Isotopes. John Wiley and Sons, New York.

Slade, D. H. (1968), Meterology and Atomic Energy, National Technical Information Service, Springfield, Virginia.

Strenge, D. L., W. E. Kennedy, Jr., and J. P. Corley (1982), Environmenta1 Dose Assessment Methods for Norma l Operations at DOE Nuclear Sites. Technical Report PNL-4410, Pacific Northwest Laboratory, Richland, Washington.

Ti11, J. E. and H. R. Meyer (1983), Radiological Assessment. National Technical Information Service," Springfield," Virginia.

Zimmerman, M. G., and D. H. Thomsen (1975), A Shielding Calculational System for Plutonium. Technical Report BNWL-1855, Pacific Northwest Laboratories, Richland, Washington. 
RHO-HS-ST - 5 P

APPENDIX A

RADIONUCLIDE DECAY PROGRAMS - "DK" AND "DK*" 



\section{USER INSTRUCTIONS: "DK"}

A. Load "DK" into the calculator (three cards), execute SIZE 225 or less. If nuclide data stored on magnetic cards will be used, SIZE according to what is on the cards then read the cards. Program "DK" will run with or without a printer attached.

B. Press R/S to begin program execution. The program permits three methods to build and use a nuclide data base.

1. Manually enter the nuclide data as it is prompted by the calculator. Enter the initial activities for each nuclide. Finally, enter the decay time and allow the program to compute the final activities.

2. Read nuclide data from magnetic cards. Manually enter new initial activities. Then enter a decay time.

3. Read nuclide data from cards and use the initial activities stored on the cards. Enter a decay time only.

On the flowsheet, the first option is presented with the "NEW SOURCE?" prompt. If only $R / S$ is pressed, the second option is offered by means of the "NEW ACTIVITY?" prompt. If no entry is made and only R/S is pressed, the program goes to the third option and prompts for a decay time.

C. Manual input of nuclide data is performed in the order described here. The program is designed to handle only single- and two-step decay chains. The number of nuclides which can be entered ranges up to 62 or 73, depending on the relative number of single and double decays.

1. At the "NEW SOURCE?" prompt, enter any number and press "R/S". Alphanumeric labels and half-lives of nuclides that decay to stable daughters are requested first. The "NAME 1" prompt refers to the alphanumeric identification of the first nuclide, such as $134 \mathrm{Cs}$. Nuclides that are part of two-step decay chains, such as $90 \mathrm{Sr}$ and $90 \mathrm{Y}$, should be entered later unless the user is confident that equilibrium is effectively reached (i.e., the ratio of the parent activity to the daughter activity is fixed, and both appear to decay with the half-life of the parent).: If no single decay nuclides will be entered, press $R / S$ and the program will go directly to the two-step decay chain prompts.

2. For each nuclide, the alphanumeric identification is limited to a six-character maximum. The half-life is next, and is followed by a unit prompt. The available units are seconds (1), minutes (2), hours (3), days (4), or years (5). The program converts these to hours, computes a decay constant, and stores it together with the name. 
3. When all single-decay nuclide data has been entered, the program will prompt for the next name. Simply press $R / S$ and the program will begin with the two-step decay prompts. The first such prompt is "NAME PARENT" which refers to the alphanumeric identification of the first nuclide in a two-step decay chain. Possible types of decay chains are shown below.

a. $\underset{\text { parent }}{\mathrm{A}} \stackrel{\lambda_{1}}{\longrightarrow} \underset{\text { daughter }}{\mathrm{B}} \stackrel{\lambda_{2}}{\longrightarrow} \underset{\text { stable }}{\mathrm{C}} \quad$ B.R. $=1.000$

b.

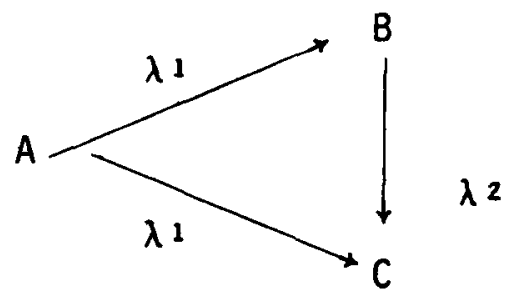

NOTE: $B . R$ is the fraction of the decays of nuclide $A$ in which nuclide $B$ is the daughter.

(1-B.R.) is the fraction of the decays of $A$ for which $C$ results.

An example of "a" is ${ }^{90} \mathrm{Sr} \longrightarrow 90 \mathrm{Y} \longrightarrow 90 \mathrm{Zr}$

An example of "b" is

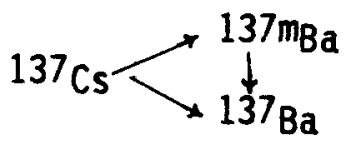

B.R. $=.946$

4. If no two-step chains will be entered, press only R/S; the program will go to the activity prompts. Otherwise, enter the name of the parent. After the parent half-life (and time unit) has been entered, the branching ratio of the parent to radioactive daughter will be prompted. Enter the correct value and press R/S. Last of all, enter the daughter identification and half-life.

5. When the final daughter is entered, the "NAME PARENT" prompt will again appear. Press only $R / S$; the program will begin prompting for the initial activities of each nuclide. If the initial activity of any isotope is zero, simply press $R / S$; zero will automatically be entered. Isotopes with zero activity will not be displayed (or printed, if the printer is attached).

6. Once the activity of the last isotope in the data base has been entered, the program will offer the option of recording this data base on cards. If desired, enter any number and press R/S. The calculator will prompt for the cards, track by track. After writing the cards, the program displays the memory size requirement for the data base. Record this on the cards for later reference. 
D. The final section of the program computes the activities remaining after a specified decay time. When the "DECAY TIME?" prompt appears, enter the desired decay period. The decay time prompt is followed by the unit menu. As before, select seconds, minutes, hours, days, or years. The final activities are computed and displayed/printed. Zero values of activity are neither displayed or printed. The formulas used to compute final activities are as follows:

$$
\begin{aligned}
& A_{1}=A_{10} e^{-\lambda 1 t} \\
& A_{2}=A 20 e^{-\lambda 2 t}+\frac{\left(B . R_{1}\right) A_{10} \lambda_{2}}{\lambda^{2}}\left(e^{-\lambda 1 t}-e^{-\lambda 2 t}\right) \quad \lambda_{1} \neq \lambda_{2} \\
& A_{2}=A_{20} e^{-\lambda 2 t}+\left(B . R_{1}\right) A_{10} \lambda_{2} t e^{-\lambda 2 t} \quad \lambda_{1}=\lambda_{2}
\end{aligned}
$$

Note that negative decay times may be entered to compute the activities of some earlier time.

INSTRUCTIONS AND LISTING - "DK*"

"DK*": Common Multiplier for the "DK" Data Base

This program is designed to multiply each of the activities stored in the "DK" data base by a common number. User instructions are as follows.

1. Press GTO.. to pack memory; enter the card containing "DK^."

2. To run "OK*," press R/S or XEQ "OK*."

3. At the "FACTOR = "prompt, enter the common multiplier and press $R / S$. In the absence of keyboard input, the multiplier used is unity. 
4. If a printer is attached, the factor is printed along with each nonzero activity.

5. The last line of "DK*" returns it to "DK" where execution continues with the "NEW SOURCE?" prompt. If "DK*" will be used repetitively, the last line, GTO "DK," should be deleted.

NOTE: The constant multiplier is in the $X$ register, the indirect address for the activities is in the $Y$ register. Memory and flag usage is the same as it is in "OK."

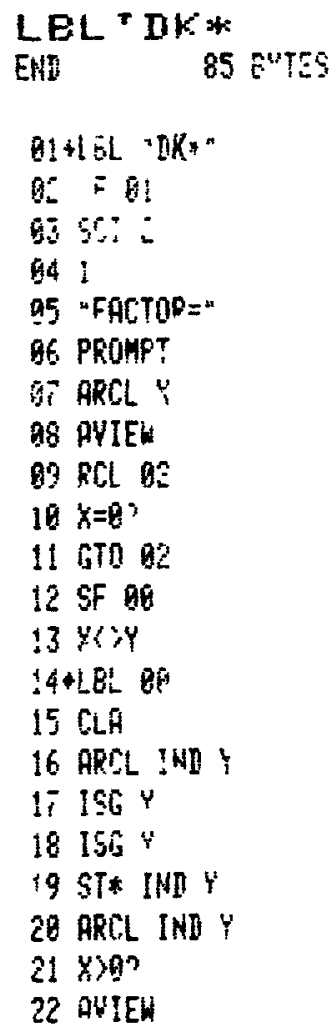

23 FS? 96

24 GTO 61

$25 \mathrm{FS} ? \mathrm{C}$ : 1

26 GTO

27 ISG $Y$

28 SF 91

29+LRL 1

39 IST Y

3167006

72 FC? 80

33 GTO 83

34 ENTER:

35 \&BL 82

36 XYYY

$37 \mathrm{RCL}$ B3

$38 x=9$ "

39 GTO 83

49 XX>Y

41 GTO

42+LBL 93

43 ADV

$44 \mathrm{GT}$ - $\mathrm{gr}$ *

45 EN? 
SAMPLE PROBLEM

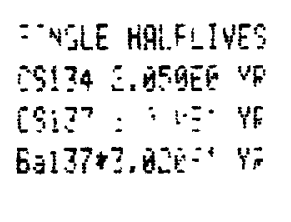

THO STEF HALFLIYTSRgG 2 . BAE: $P$

$E_{.} E_{2}=1$.

$\because 906.46981 \mathrm{hr}$

26.95 6.460E1 in:

B. $R_{0}=1.00090$

$146953.510 \mathrm{El}$ int

\section{CURIES}

CS134 1. 8QAE:

CS137 5.0005

Ea137*4.736E0

SR.98 2. $968 E$ !

Y 90 i. 9 GQE

$2995 \quad 6.90901$

Whys 8.9095

$\because n=3$

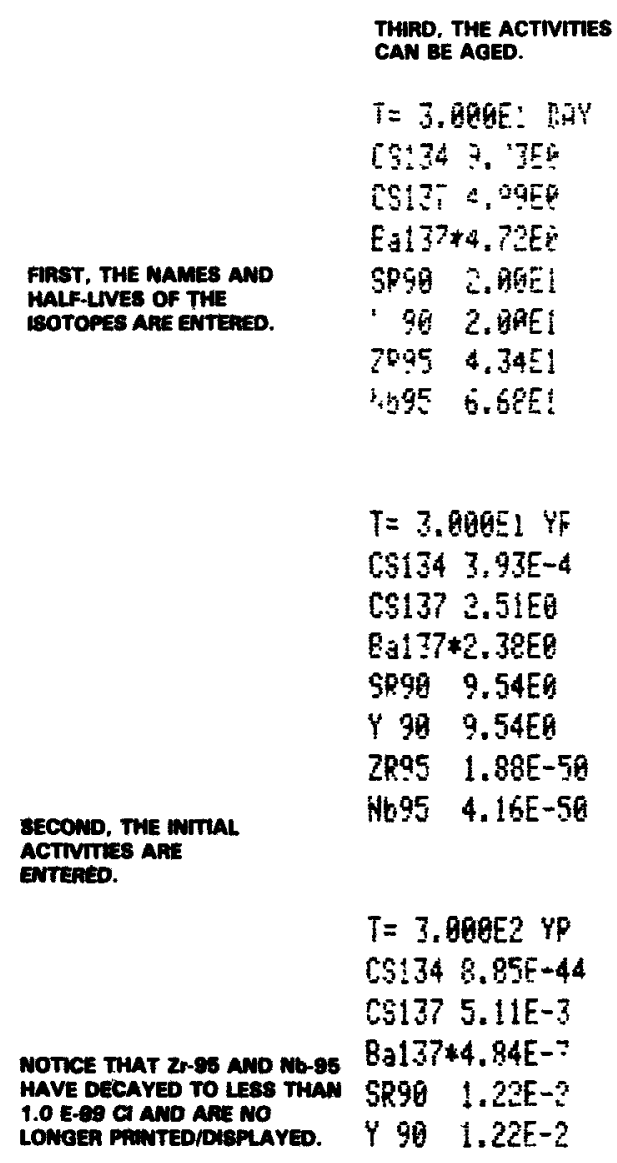

FACTOP $=-\therefore, A Q C A$

CS134 ㅐㄷ:

MIZ?

DAIJT*-, - EE

SRge 4. 395

Y 9h ?.8BE:

2Fys $1.20 \mathrm{E} 2$

Hb95 $1.60 \mathrm{E} 2$ 


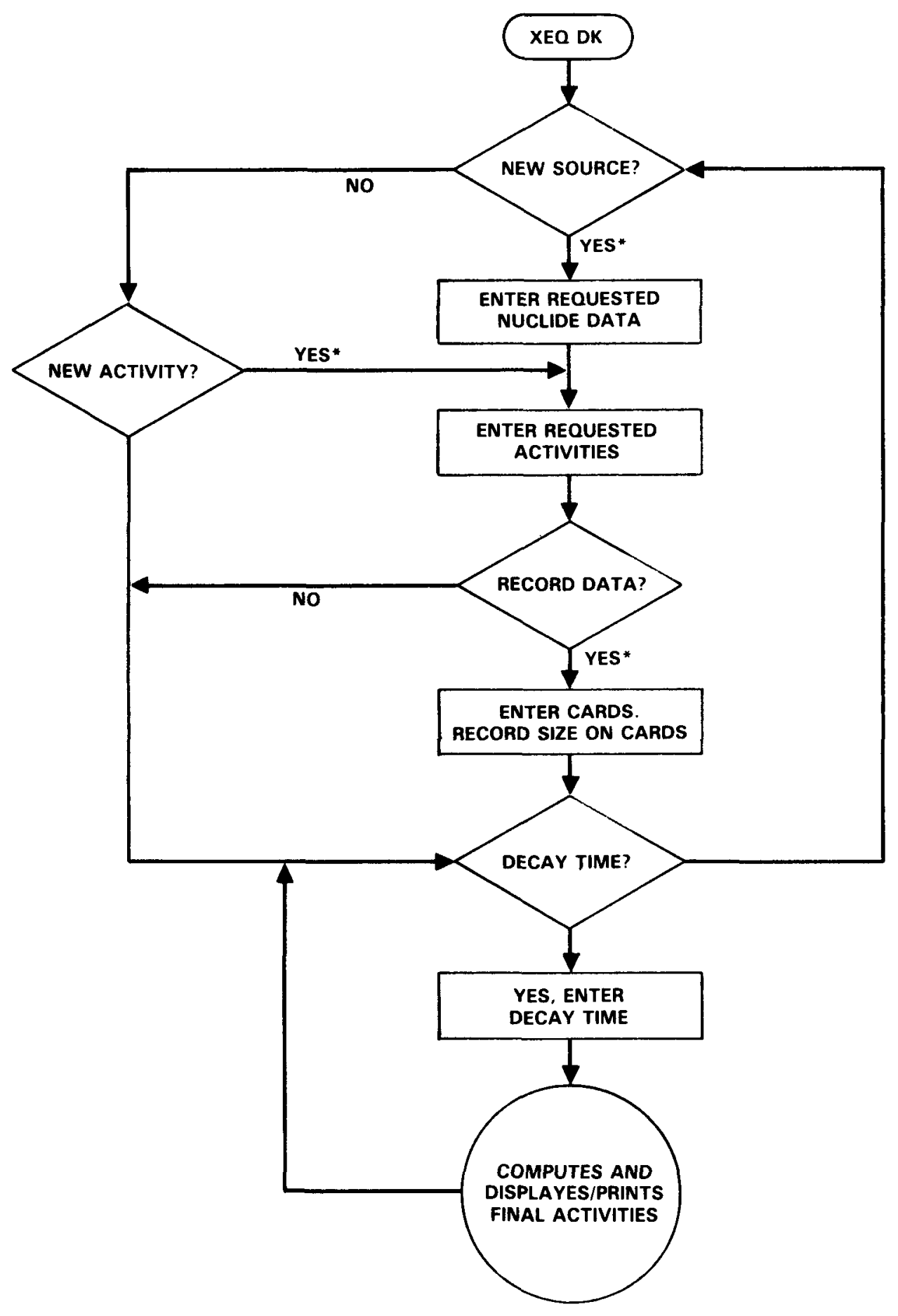

*YES FLOW PATHS ARE OBTAINED BY ENTERING ANY NUMBER AND PRESSING R/S. PRESSING R/S ALONE CAUSES EXECUTION ALONG THE NO FLOW PATHS.

FIGURE 1. Flowsheet for Program "DK." 
PROGRAM LISTING

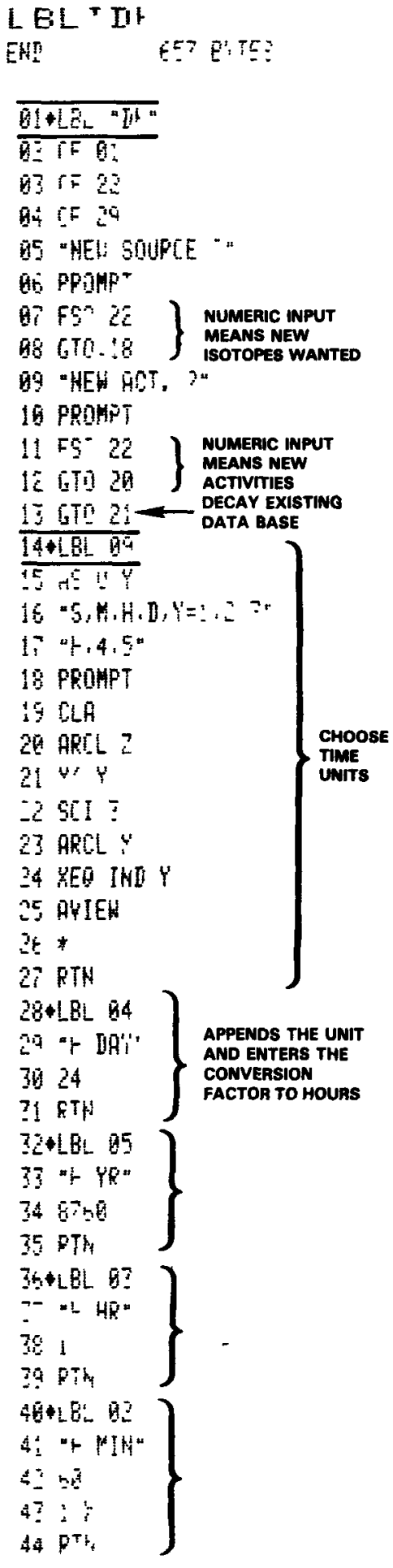

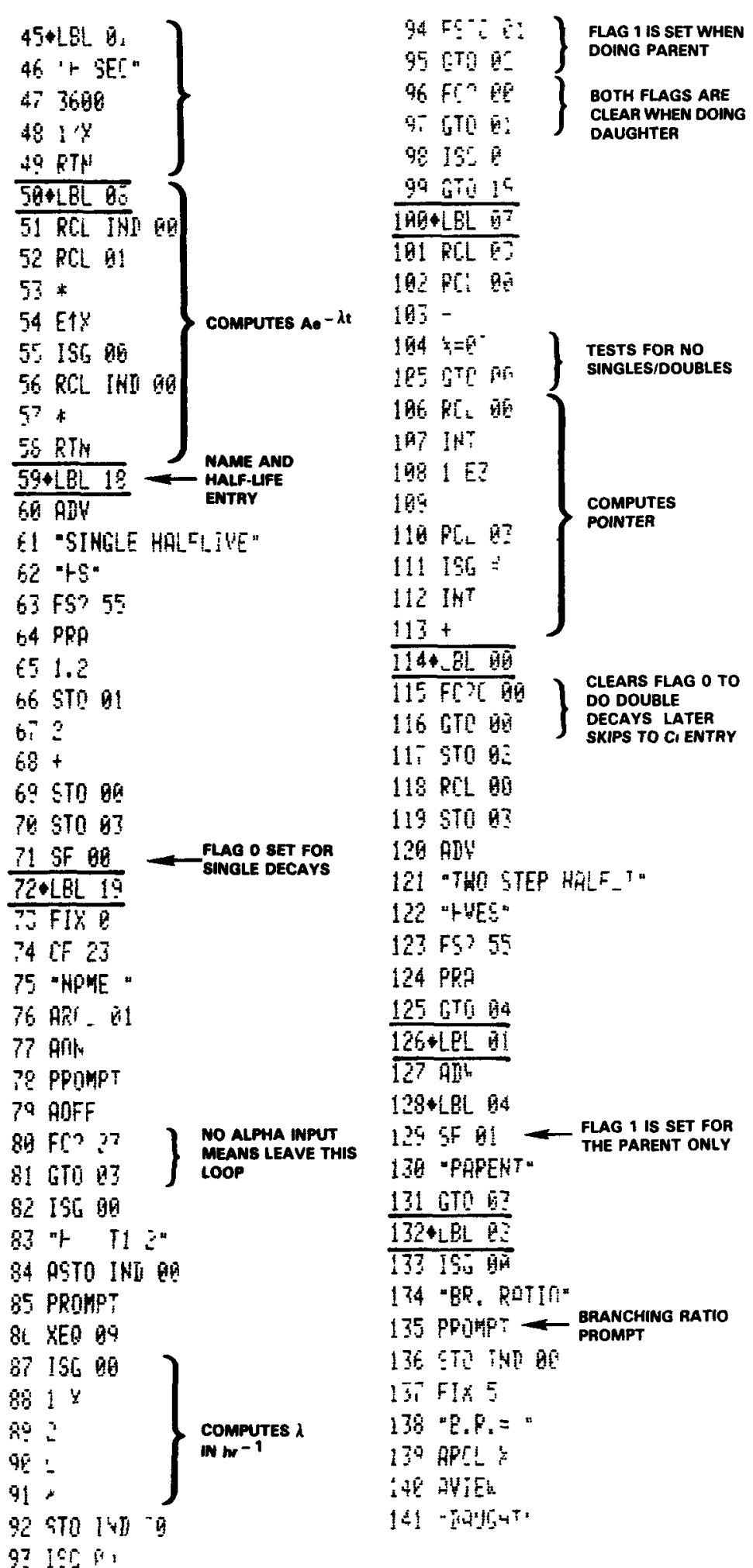


PROGRAM LISTING

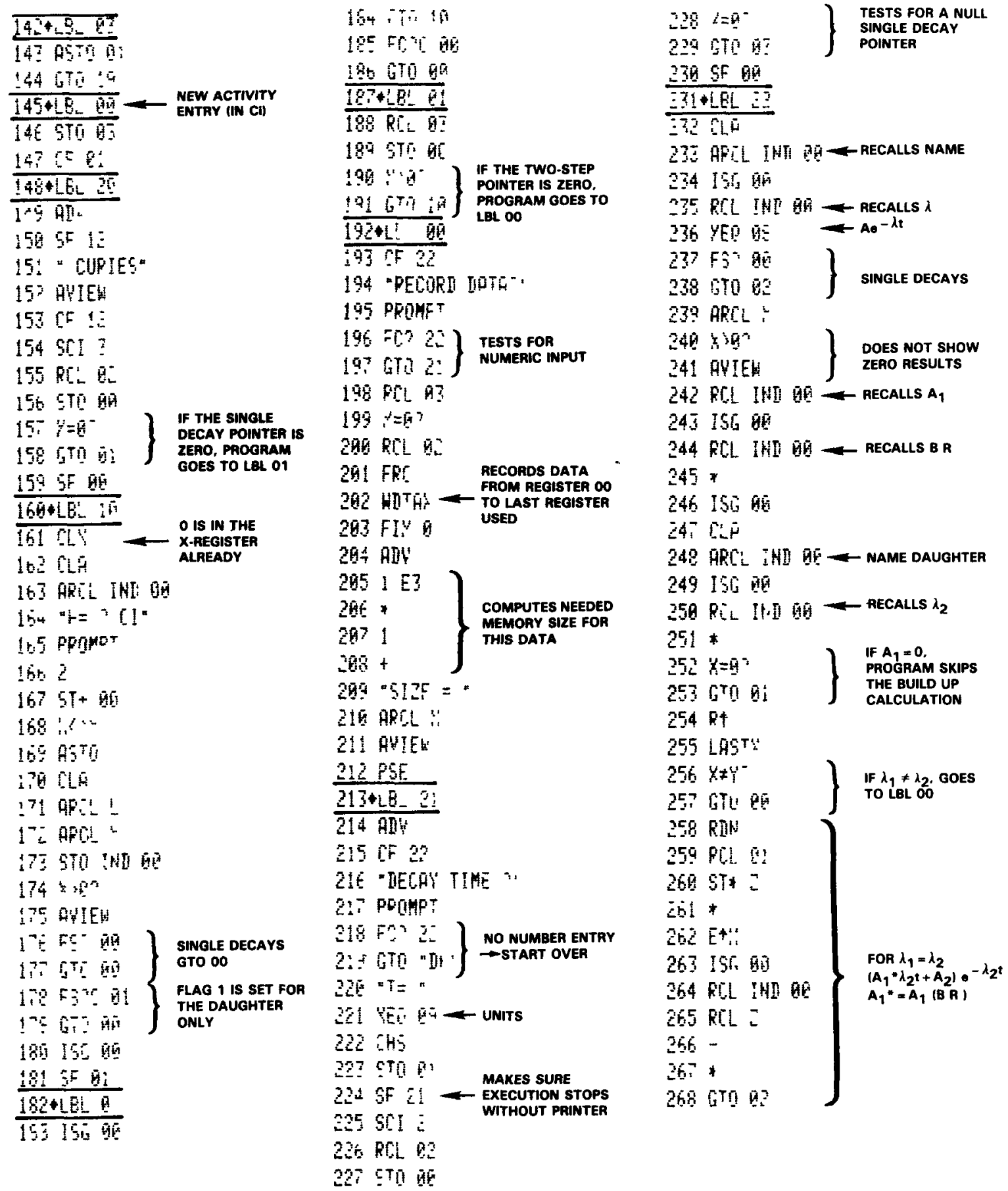




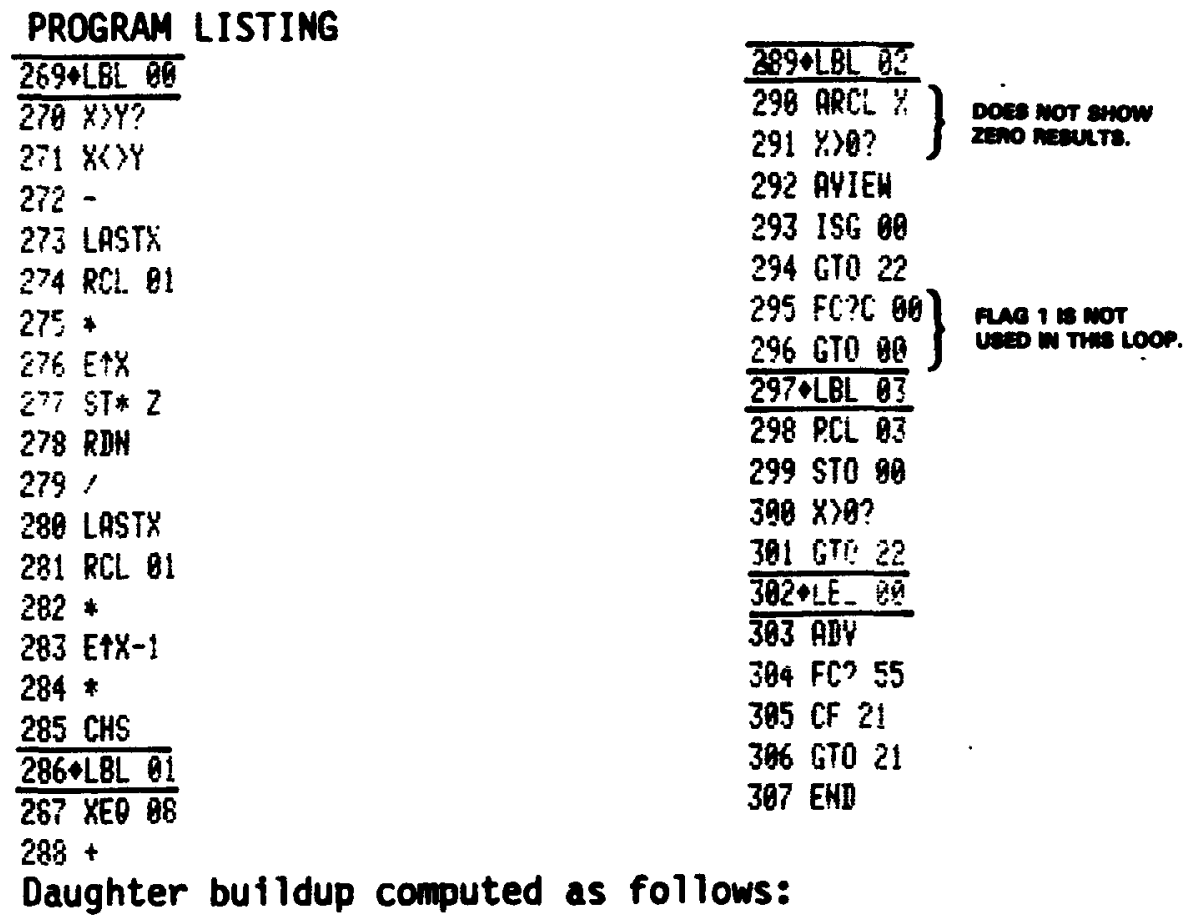

$$
\begin{aligned}
& \lambda_{1}>\lambda_{2}: \frac{A_{1} \star \lambda_{2} e^{-\lambda_{2} t}}{\lambda_{1}-\lambda_{2}} \quad\left[e^{-\left(\lambda_{1}-\lambda_{2}\right) t}-1\right] \\
& \lambda_{2}>\lambda_{1}: \frac{A_{1} \star \lambda_{2} e^{-\lambda_{1} t}}{\lambda_{2}-\lambda_{1}}
\end{aligned}
$$

MEMORY USE:

00: Pointer in use

01: Negative decay time

02: Single-decay pointer

03: Two-step pointer
FLAG USE:

00: Single-decay nuclides

01: Two-step decays 
RHO-HS-ST-5 P

APPENDIX B

PLUTONIUM PRQGRAMS - "PU" and "NT"

$B-1 / 2$ 

RHO-HS-ST-5 P

\section{USER INSTRUCTIONS}

A. Load "PU" by executing SIZE 081, reading in the data cards ( 3 tracks), and finally reading in the program cards (10 tracks). The printer is required for program execution. Place the print mode switch in the "MAN" position.

B. XEQ "PU" (or just press R/S after reading the cards). The first prompt, "\%, M, OR AC ?," requires the user to inform the program whether the initial data to be entered is the weight percents, masses, or activities of the various isotopes. Use the alpha keyboard to enter either "\%", "M", or "AC." Press R/S; the program will begin prompting for the appropriate data entries. Enter the data requested and press $R / S$. Zeroes will automatically be entered if no other number is input.

C. After printing tables of the initial percent, mass, and activities; the program prompts for a decay time in years. If no decay is desired, enter nothing; press R/S. If decay is desired, enter the decay time, in years, and press R/S. After printing tables of the final percent, masses, and activities; the program prompts for a second decay time. If a second set of tables is desired, enter the appropriate number of years and press $R / S$. (The decay time may be negative to compute masses and activities of an earlier date.) If a second decay time is not desired, simply press $R / S$.

D. The next option allows one to keep the initial weight percents, but adjust the total mass of Pu plus Am. To change the total mass, enter the new mass and press R/S. To go to the next option, enter nothing; press $R / S$.

E. The program now asks where to go next. The menu is displayed: "PU, AGE, M2, NT."

"PU" - Starts the program from the beginning (see instruction A).

"AGE" - Prompts for a decay time to age the source (see instruction D).

"M2" - Prompts for a new total mass (see instruction E).

"NT" - Permits computation of neutron production rates for various compounds; however, this program is separate from the present, and must be loaded into the calculator (see instruction F).

F. User Instructions for "NT."

1. Press GTO.. to pack memory; enter the two cards containing "NT." It is not necessary that "PU" be in the calculator, but it is imperative that "PU" has been run so that the proper data base has been created. 
2. XEQ NT and the program prompts for the chemical form of the plutonium: "02, N03, F4, C, Be?". To request oxide, for example, enter " 0 " and "2" from the alpha keyboard and press R/S. If bare metal is of interest, enter any chemical form and just ignore the alpha-neutron result in the neutron production rate.

3. The program returns to the chemical form menu. If no additional forms need to be computed, press $R / S$ and the program will return to the "PU" program at the menu prompt discussed in instruction $E$. 


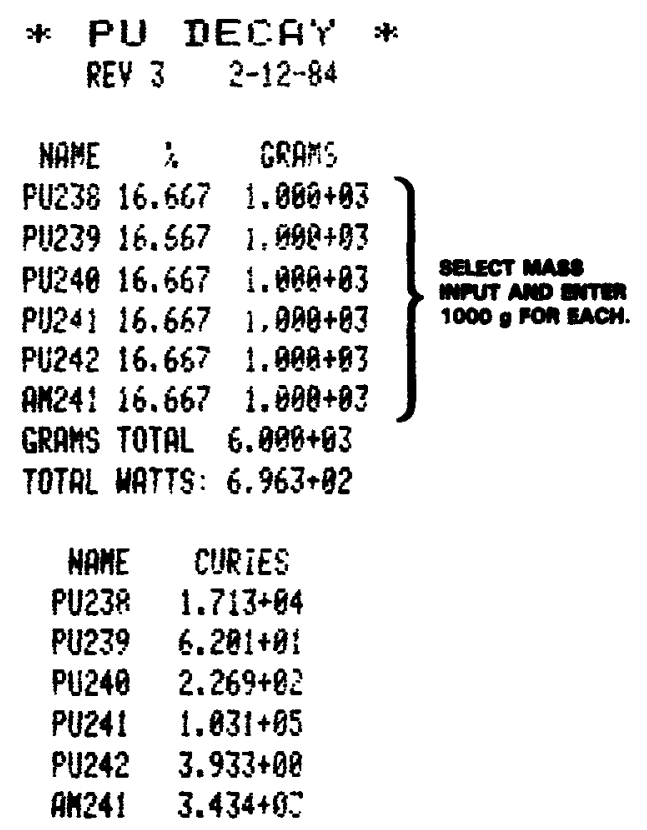

TOTAL ALPHA: $2,685+04 \mathrm{CI}$

TOTAL BETA: $1.83 \mathrm{i}+95 \mathrm{CI}$

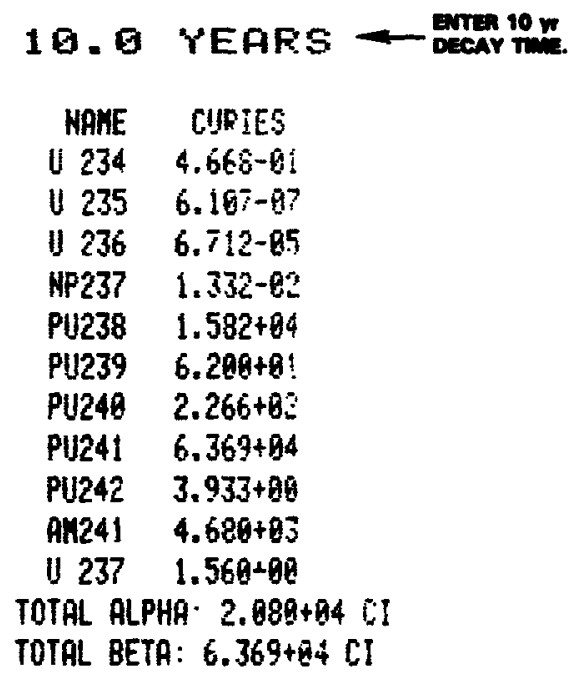

\begin{tabular}{|c|c|c|}
\hline NAME & $\%$ & CRAYE \\
\hline U 234 & 1.245 & $7.467+8$ \\
\hline U 235 & 0.865 & $2.8: 4-91$ \\
\hline |1 236 & 0.617 & $1.677^{2}+90$ \\
\hline UP237 & 0.315 & $1.888+91$ \\
\hline FL238 & 15.485 & $46+92$ \\
\hline P11239 & 16.667 & $9.997+82$ \\
\hline PUI 240 & 16.6 .54 & \\
\hline
\end{tabular}

PU241 $10.302 \quad 6.179+82$

PU242 16.571 1.006+93

Anl241 $22.720 \quad 1.36 .3+133$

GRAMS TOTAL $5.998+93$

TOTAL HATTS: $6.93 \mathrm{~A}+82$

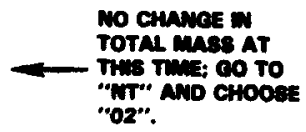

PuDz n-́sec.

$\alpha-n \quad 1.625+E^{\circ}$

S-F $\quad 5.344+8$

Tot a 1 2.161+8?

AFTER 10.9 YEPDS

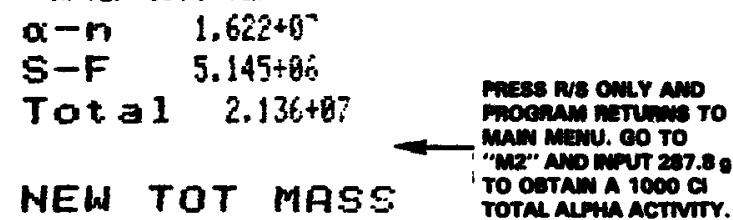

HAME \% GRAMS

PU238 $16.667 \quad 4.797+81$

PU239 16.667 4.757+9t

PU248 $16.667 \quad 4.797+91$

PU241 16.667 $4.797+91$

PU242 $16.867 \quad 4.797+91$

AN241 $16.667 \quad 4.747+1$

GRAMS TOTAL $2.878+92$

TOTAL WATTS: $3.340+91$

$\begin{array}{rc}\text { NAME } & \text { CURIES } \\ \text { PU238 } & 8.215+0 ? \\ \text { PU239 } & 2.975+90 \\ \text { PU240 } & 1.688+01 \\ \text { PU241 } & 4.943+03 \\ \text { PU242 } & 1.886-01 \\ \text { AM241 } & 1.647+62\end{array}$

TOTAL ALPUS: $1.999+9.3 \mathrm{C}: 1<-1000$ a TOTAL TOTAL BETA: $4.943+B 3$ CT

$$
\begin{array}{ll}
\text { PuF4 } & \text { ns sec } \\
\alpha-n & 1.189+95 \\
\text { s-F } & 2.564+85 \\
\text { Total } & 1.192+98
\end{array}
$$




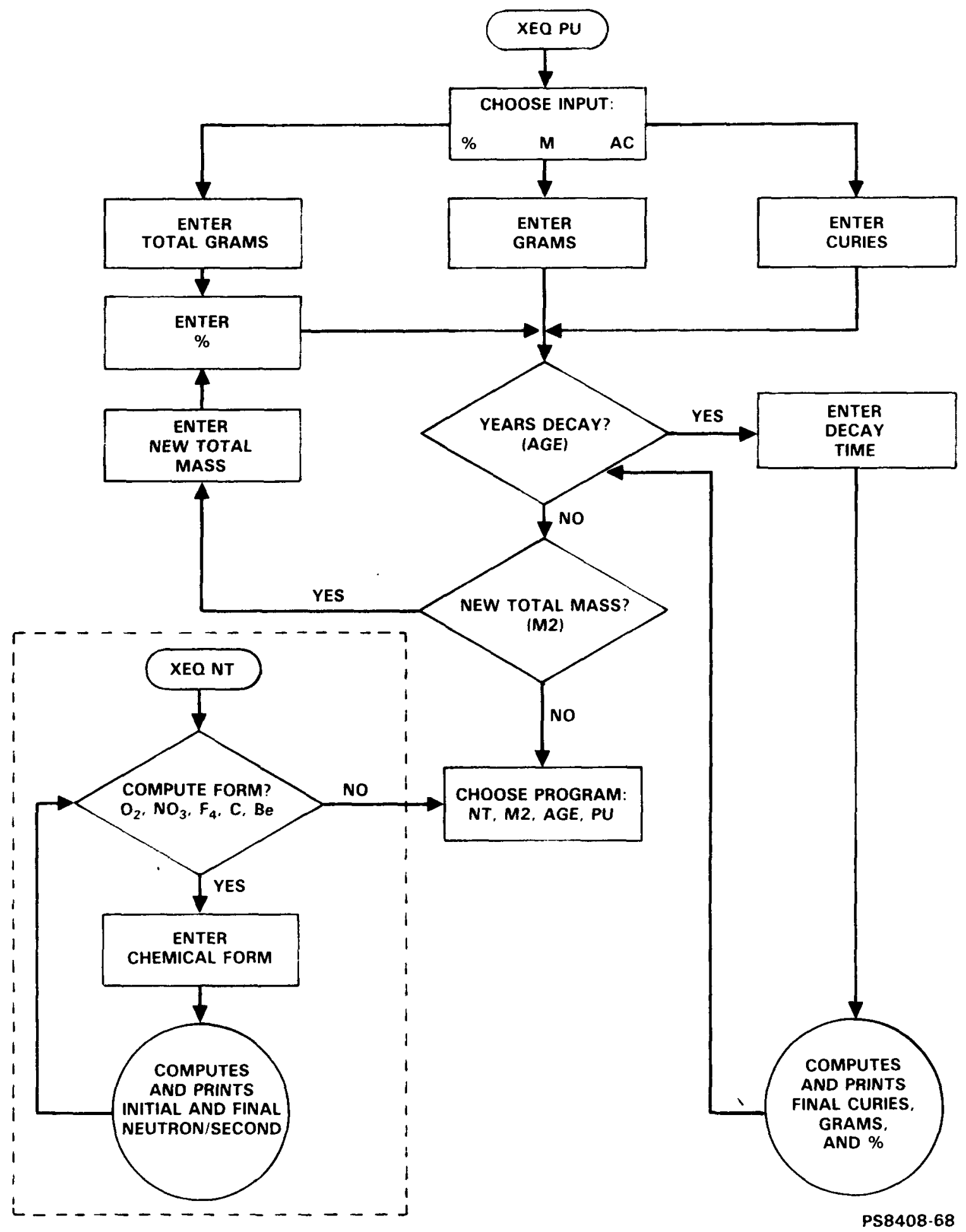

FIGURE 2. Flowsheet for Program "PU." 
TABLE 1. Isotope Data Used by "PU".

\begin{tabular}{|c|c|c|c|c|c|c|c|}
\hline Isotope & $\begin{array}{c}\text { Isotopic } \\
\text { mass }\end{array}$ & Half-life & $\% S^{*}$ & $\mathrm{Ci} / \mathrm{g}$ & $\mathrm{g} / \mathrm{Ci}$ & $W / g$ & $\mathrm{~W} / \mathrm{Ci}$ \\
\hline $238 \mathrm{pu}$ & 238.052 & $8.775 E+01 y r$ & $1.84 \mathrm{E}-07$ & $1.713 E+01$ & $5.839 E-02$ & 54.687 E-02 & .0332 \\
\hline $234 U$ & 234.041 & $2.445 \mathrm{E}+05 \mathrm{yr}$ & $0.12 E-08$ & $6.252 \mathrm{E}-03$ & $1.600 \mathrm{E}+02$ & $1.801 E-04$ & .0288 \\
\hline $239 \mathrm{Pu}$ & 239.052 & $24.131 \mathrm{E}+03 \mathrm{yr}$ & 0.44 E-09 & $6.201 E-02$ & $1.613 E+01$ & $1.929 E-03$ & .0311 \\
\hline $235 U$ & 235.044 & $7.038 E+08 y r$ & $0.20 \mathrm{E}-06$ & $2.163 E-06$ & $4.624 E+05$ & 5.818 E-08 & .0269 \\
\hline $240 \mathrm{pu}$ & 240.054 & $6.569 E+03 y r$ & $4.95 E-0.6$ & $2.269 E-01$ & $4.408 E+00$ & $7.055 E-03$ & .0311 \\
\hline $236 U$ & 236.046 & $23.414 \mathrm{E}+06 \mathrm{yr}$ & $0.12 E-06$ & $6.472 E-05$ & $15.450 E+03$ & 1.754 E-06 & .0271 \\
\hline $241 \mathrm{Pu}$ & 241.057 & $0.144 E+02 y r$ & & $1.031 E+02$ & $9.703 E-03$ & $0.423 E-02$ & .000041 \\
\hline $237 U$ & 237.049 & $0.675 E+01$ day & & $8.16 \quad E+04$ & $1.225 \mathrm{E}-05$ & $0.384 E+03$ & .0047 \\
\hline $241_{A m}$ & 241.057 & $4.322 E+02 y r$ & $3.77 E-10$ & $3.434 E+00$ & $2.912 \mathrm{E}-01$ & $1.143 E-01$ & .0333 \\
\hline $237_{N p}$ & 237.048 & $0.214 E+07$ & 0.20 E-09 & 7.052 E-04 & $1.418 E+03$ & $2.024 \mathrm{E}-05$ & .0287 \\
\hline $242 \mathrm{pu}$ & 242.059 & $3.758 E+05 y r$ & $0.55 E-03$ & 3.933 E-03 & $2.543 E+02$ & 1.160 E-04 & .0295 \\
\hline $238 U$ & 238.051 & $4.468 E+09 y r$ & $0.55 E-04$ & 3.363 E-07 & $2.973 E+06$ & $0.851 E-08$ & .0253 \\
\hline
\end{tabular}

$\star \% S F=$ the percent of decays that undergo spontaneous fission. 
TABLE 2. Neutron ( $n$ ) Yields Used by "NT" Program.

\begin{tabular}{|c|c|c|c|c|c|c|c|c|c|c|c|}
\hline Isotope & \multicolumn{2}{|c|}{ Spontaneous } & \multicolumn{2}{|l|}{ Oxide } & \multicolumn{2}{|c|}{ Fluoride } & \multicolumn{2}{|c|}{ Carbide } & \multicolumn{2}{|c|}{ Beryllium } & $\begin{array}{l}\text { Alpha } \\
\text { energy } \\
\text { (MeV) }\end{array}$ \\
\hline $\begin{array}{l}239 \mathrm{pu} \\
235 \mathrm{u}\end{array}$ & $\begin{array}{l}0.28 E-01 \\
0.33 E-03\end{array}$ & $\begin{array}{l}0.45 E+00 \\
1.53 E+02\end{array}$ & $0.380 E+02$ & 613 & $63.50 E+02$ & $1.024 E+05$ & $0.373 E+02$ & 601 & $1.36 E+05$ & $2.192 E+06$ & $\begin{array}{l}5.15 \\
4.4\end{array}$ \\
\hline $\begin{array}{l}240 \mathrm{pu} \\
236 \mathrm{U}\end{array}$ & $\begin{array}{r}10.20 E+02 \\
0.58 E-02\end{array}$ & $\begin{array}{l}45.00 E+02 \\
0.90 E+02\end{array}$ & $0.145 \varepsilon+03$ & 639 & $23.50 E+03$ & $1.036 E+05$ & $0.138 E+03$ & 608 & $5.02 E+05$ & $2.213 E+06$ & $\begin{array}{l}5.17 \\
4.49 \\
\end{array}$ \\
\hline $\begin{array}{l}242 \mathrm{pu}_{\mathrm{u}} \\
241_{\mathrm{Am}}\end{array}$ & $\begin{array}{r}17.00 E+02 \\
0.11 E+01\end{array}$ & $\begin{array}{l}4.33 E+05 \\
0.33 E+00\end{array}$ & $\begin{array}{l}0.213 E+01 \\
2.680 E+03\end{array}$ & $\begin{array}{l}542 \\
782\end{array}$ & $\begin{array}{l}2.83 E+02 \\
4.09 E+05\end{array}$ & $\begin{array}{l}7.205 E+04 \\
1.190 E+05\end{array}$ & $\begin{array}{l}0.209 E+01 \\
2.980 E+03\end{array}$ & $\begin{array}{l}532 \\
869\end{array}$ & $\begin{array}{r}60.70 E+02 \\
8.77 E+06\end{array}$ & $\begin{array}{l}1.543 E+06 \\
2.555 E+06\end{array}$ & $\begin{array}{l}4.90 \\
5.49\end{array}$ \\
\hline
\end{tabular}




\section{PROGRAM LISTING}

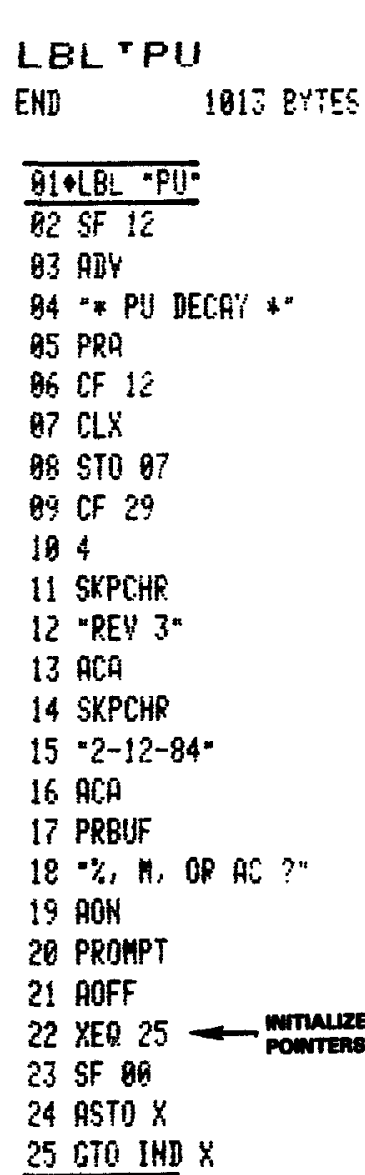

$\frac{26+L B L}{27 H^{2}}-$ mass mpurs. $27 \mathrm{CLX}$

28 STO 68

29+LBL 96

30 -CRANS -

31 ARCL IND 90

32 PROAPT

33 STO IND 84

$34 \mathrm{ST}+88$

35 ISC 99

$36 \mathrm{CLX}$

37 IS6 94

38 GTO 60

$39 \times E Q 25$

40 KEQ 28

4167021

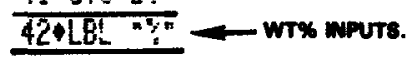

43 All

$44 \mathrm{CLF}$

45 AFI 66

46. "GGRAMS?"

47 PROMPT
4851098

$49 \mathrm{CLX}$

50 STก 65

$51 \mathrm{FI} 3$

$52+261$ पो

53 . \% .

54 ARCL INI 96

55 PROMPT

56 STO IHD 03

$575 T+95$

58 ISG 80

$59 \mathrm{CLX}$

$69156 \quad 93$

61 GTO B1

62.

63 ARCL 86

64 " $\% \%$ :

65 ARCL 95

66 PRA

670LBL 20

68 XEQ 25

69 XEQ 28

$79 \mathrm{CLX}$

71 STO 95

72+LBL 82

73 FIX 3

74 CLA

75 ARCL IND 96

76 RCL 88

$77 \mathrm{RCL}$ INB 93

7810

$79 \times 1 Y$ ?

80 if -

B1 RDH

82 ARCL $:$

83 "t "

$64 \%$

85 STO IND 84

86 SCI 3

87 XEG 14 - PAwts taduE.

$88 \mathrm{RCL}$ IND $\mathrm{Q}$

$89 *$

$90 \mathrm{ST}+65$

91156 of

92 ISG 02

g? ISG 83

$94 \mathrm{CLX}$

9515604

96 GTO B2

97 XEE 2 ?

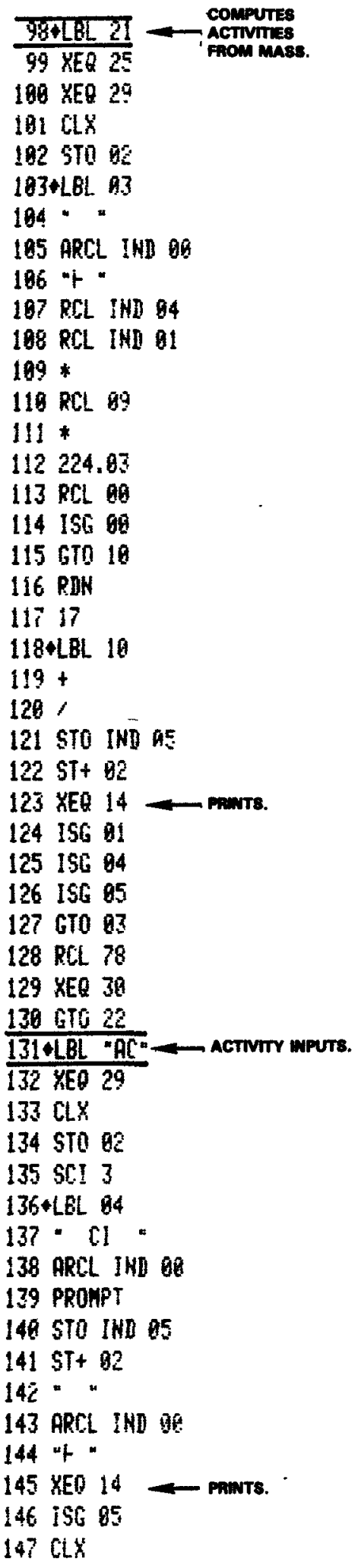


RHO-HS-ST-5P

\section{PROGRAM LISTING}

$148 \mathrm{CA}$

149 ISC 98

15967094

$151 \mathrm{RCL} 78$

152 YEQ 39

153 XEO 25

154 XEQ 31

155 XEQ 25

156 XEO 26

$157 \cdot L B L 22$

1594LBL "AGE"

159 ADY

160 CF 2 ?

161 "YERRS DECAI?"

162 PROMPT

163 FC? 22

164670 " $\mathrm{n}$ "

165 CF 60 - PAO DO. CLEANED

166 FIX 1

16795

$168 \mathrm{XY}$ ?

$169 . F I X$

170 RDN

171 ADY

172 ASTO L

173 CLA

174 SF 12

175 ARCL $\%$

176 it -

177 ARCL L

178 PRg

179 CF 12

180 CHS

181 \$TO 97

182 XEQ 25

$183 \mathrm{SCl} 3$

18410.01

$185 \mathrm{ST}+02$

186*LBL 65

$187 \mathrm{RCL}$ IHD 01

$188 \mathrm{RCL}$ : ?

$184 *$

$190 \mathrm{E}+\mathrm{X}$

$191 \mathrm{RCL}$ IND 85

$192 *$

193 STO IND Q2

194 ISE $8 !$

195156 ?

196 ISG BS

197 GTO 85

$198 \mathrm{PC} 3$

$199 \mathrm{RC} .99$
$206 *$

201 STO $52=4$ U.237

$202 \mathrm{RCL} 35$

$203 \mathrm{RCL} 24$

264 XEQ $2 ?$

265 STO 45

$206 \mathrm{RCL} 28$

207 RCL 24

208 XEQ 23

209 RCL 45

219 -

211 CHS

212 RCL 28

$213 \mathrm{RCL} 36$

214 -

215 LASTY

216 :

217 ,

$218 \mathrm{RCL} 78$

$219 *$

228 RCL 45

221 RCL 8 C

$222 *$

$223+$

224 STO $45-4$ A 15 -237

225 RCL 25

$226 \mathrm{RCL} 21$

227 XEQ 23

$228 \mathrm{RCL} 75$

$229 *$

236 5TO $42-A_{4}$ U.234

231 RCL 26

$232 \mathrm{RCL} 22$

233 XEO 23

$234 \mathrm{RCL} 76$

$235 *$

236 STO $43-4,4-238$

$237 \mathrm{RCL} 27$

238 RCL 23

239 YEQ 23

$246 \mathrm{RCL} 77$

$241 *$

242 STO $44 \longrightarrow 4$ U-238

243 RCL 28

244 RCL 35

245 XEQ 23

$246 \mathrm{RCL} 78$

$247 *$

$248 \mathrm{ST}+51=A_{1} \mathrm{Am}_{\mathrm{m}-241}$

249 KES 29

250 XED 24

251.081 
RHO-HS-ST-5 P

PROGRAM LISTING

$25 \hat{2} 57+89$

$25.3 \mathrm{CLX}$

$254 \$ 1092$

255+LBL at.

256 "

257 ARCL IND AQ

258 "F -

259 RCL IND 95

260 XEP 14

$261 \mathrm{ST}+62$

$26 \hat{2}$ 15G 80

$26.5 \mathrm{CL}$

264 ISG 85

265 GT0 96

$266 \mathrm{RCL} 52$

$267 \mathrm{RCL} 49$

$268+$
$269 \times 130$

270 XED 24

271 XEO 31

$272 \times E 024$

273 XEQ 26

274 GTO 22

275 ADY

276.LBL $-122^{n}$

277 CF 22

278 "HEH TOT MASE"

279 PROMPT

$289 \mathrm{FC} 22$

281 GTO 10

28257008

283 ADY

284 SF 12

285 PRA
286 CF 12

$287 \mathrm{CLX}$

$2865 T 007$

289 CTO 29

290NLBL 16

$291+181$ " $2 a "$

292 ADN

293 -PU, ACE, K2 NT=

294 AON

295 PRONPT

296 AOFF

297 ASTO $Y$

298 GTO IND $*$ Computes

$\frac{299+L B L ~ 23}{390-}-\frac{\lambda_{20}-\lambda_{2 t}}{\lambda_{1}-\lambda_{2}}\left(1-0-u_{1}-\lambda_{2} \lambda_{1}\right)$

301 LASTX

302 LASTX

393 RCL 07

Formulas Used:

$$
\begin{aligned}
& \underset{2}{\longrightarrow} \stackrel{\lambda_{1}}{\longrightarrow} \underset{3}{\stackrel{\lambda_{3}}{\longrightarrow}} \\
& A_{1}=A_{10} e^{-\lambda_{1} t} \\
& A_{2}=A_{20} e^{-\lambda_{2} t}+\frac{\lambda_{2} A_{10}}{\lambda_{2}-\lambda_{2}}\left(e^{-\lambda_{2} t}-e^{-\lambda_{1} t}\right) \\
& A_{3}=\frac{\lambda_{2} A_{10}}{\lambda_{1}-\lambda_{2}}\left[\frac{\lambda_{3}}{\lambda_{2}-\lambda_{3}}\left(e^{-\lambda_{3} t}-e^{-\lambda_{2} t}\right)-\frac{\lambda_{3}}{\lambda_{1}-\lambda_{3}}\left(e^{-\lambda_{3} t}-e^{-\lambda_{1} t}\right)\right] \\
& +\frac{\lambda_{3} A_{20}}{\lambda_{2}-\lambda_{3}}\left(e^{-\lambda_{3} t}-e^{-\lambda_{2} t}\right)
\end{aligned}
$$


RHO-HS-ST-5 P

PROGRAM LISTING

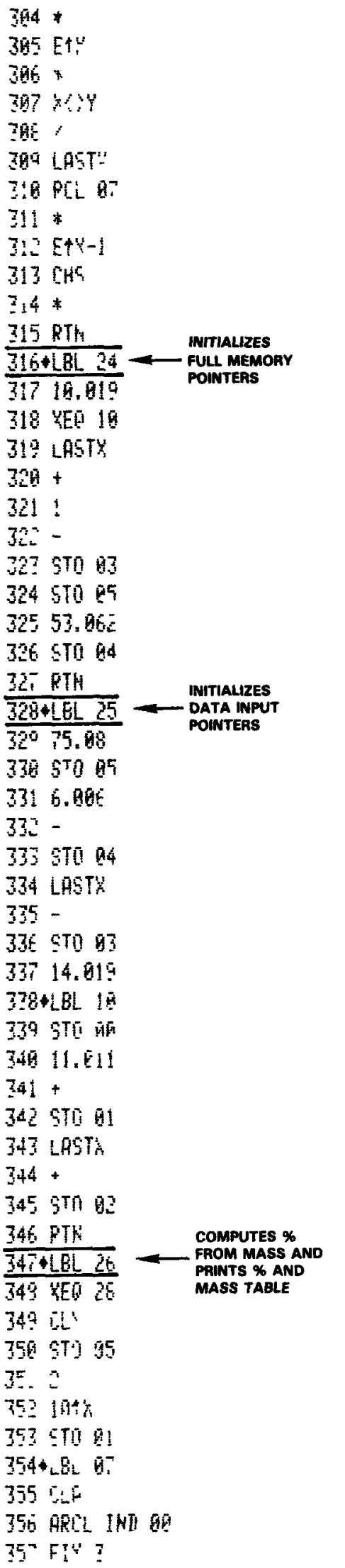

35816

754 PCL IhI 4

TEG RL

It:

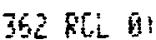

$3 \mathrm{t}^{3} *$

$364 F 52$ B9

355 STO INI 63

366 RNI

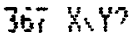

$369 " 1 "$

369.961

$379 x: 34$

$371 \% \mathrm{KH}^{7}$

$37 \mathrm{CL}$

373 APC!

37 "t

375 STl?

376 RCL IHI 94

37? XED I4

$376 \mathrm{FCL}$ IWL 92

$37 \bar{y} *$

$3845 T+65$

361 ISG

392 i56

387 196 83

384 C."

36515604

386 GTO 9 ?

$367+\angle B L 27$

398 "CRAHS "

784 aPrL 06

$399 \mathrm{RL} \mathrm{B}^{\circ}$

791 NEQ 14

$392 \mathrm{CL}$

793 ARIL 96

794 "HWATS

395 rol 85

$796+$ LBL 14

$397{ }^{4}=0^{\circ}$

398 PTk

395 AIF

$489 \mathrm{AL}^{-}$

4QI PPEUF

402 RTN

$4 B_{3}+$ LL 20

$494 \mathrm{ODW}^{4}$

40: " NAME :

$4 \bar{B}$ "BREME"

4प्? PFH

4 혀 $P T$

$4 p+B_{L}$
$4: 8 \mathrm{ADY}$

$4: 3$

412 SFPHB

$4 ! 3$-NAHE CURIE:"

414 ACP

4:5 PBBUE

416 RTN

4.74LL 34

$41 E S T-B C$

$419 \mathrm{ILA}$

420 AKCL 66

421 "FALPHÄ "

$42 C$ RCL B

$423 \times 5919$

424 CLP

455 AFCL 66

426 'PEETA =

$427 \mathrm{~K} Y \mathrm{Y}$

$438+L E L 19$

435 ACA

$430 \mathrm{ACA}$

$471=6 !$

432 ars

433 PPBUF

434 FTH

$\overline{435+\mathrm{LEL} 31}$ COMPUTES MASS

$436 \mathrm{CLF}$

437 sin of

$438+18 ! 08$

$479 \mathrm{RCL}$ INL $\mathrm{A5}$

$446 \mathrm{RCL}$ IND $\mathrm{Q}$ ?

441

$442 \mathrm{RLL} \quad 84$

443 ;

444224.93

$4 d 5$ RCI

446. IS6 94

447 GTI 19

448 PIN

44.5

$45 \overline{1 L L L} 19$

$45 !+$

452

$4539 T 0$ IHI

$454 \mathrm{ST}+98$

45519691

458 ISL 85

$45^{\circ} 156 \mathrm{~h}$

458 GTन 68

$452 \mathrm{FTH}$

4 Fin $\mathrm{E}+\mathrm{I}$ 
MEMORY USE :

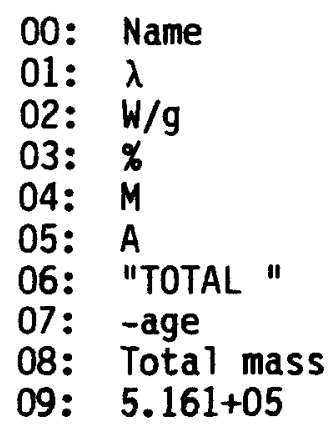

\section{LABEL 25}

$00: \quad 14.019$

01: 25.030

02: 36.041

03: 63.068

04: 69.074

05: 75.080
FLAG USE:

00: Set for data entry; cleared for aging.

01: Set to indicate $\mathrm{Pu}\left(\mathrm{NO}_{3}\right)_{2}$.

02: Set when computing aged $n / s$.

\begin{tabular}{|c|c|c|c|c|c|c|c|c|c|c|}
\hline \multicolumn{2}{|c|}{ Name } & \multicolumn{2}{|c|}{$\lambda\left(y r^{-1}\right)$} & \multicolumn{2}{|r|}{$W / g$} & $A_{f}$ & $M_{f}$ & \multirow[b]{2}{*}{ \% } & \multirow[b]{2}{*}{$M_{0}$} & \multirow[b]{2}{*}{$A_{0}$} \\
\hline $\begin{array}{l}\text { 10: } \\
\text { 11: } \\
\text { 12: } \\
13:\end{array}$ & $\begin{array}{l}\text { " } \\
\text { "U } 234 \\
\text { "U } 235 \\
\text { "U } 236 \text { "NP237 }\end{array}$ & $\begin{array}{l}\text { 21: } \\
\text { 22: } \\
23: \\
24:\end{array}$ & $\begin{array}{l}2.835-06 \\
9.849-10 \\
2.960-08 \\
3.239-07\end{array}$ & $\begin{array}{l}\text { 32: } \\
\text { 33: } \\
34: \\
35:\end{array}$ & $\begin{array}{l}1.801-04 \\
\overline{5} .818-08 \\
1.754-06 \\
2.024-05\end{array}$ & $\begin{array}{l}\text { 42: } \\
43: \\
44: \\
45:\end{array}$ & $\begin{array}{l}53: \\
54: \\
55: \\
56:\end{array}$ & & & \\
\hline $\begin{array}{l}\text { 14: } \\
\text { 15: } \\
\text { 16: } \\
17: \\
18: \\
19:\end{array}$ & $\begin{array}{l}\text { "PU238 " } \\
\text { "PU239 " } \\
\text { "PU240 " } \\
\text { "PU241 "PU242 " } \\
\text { "AM241 }\end{array}$ & $\begin{array}{l}25: \\
26: \\
27: \\
28: \\
29: \\
30:\end{array}$ & $\begin{array}{l}7.899-03 \\
2.872-05 \\
1.055-04 \\
4.814-02 \\
1.844-06 \\
1.604-03\end{array}$ & $\begin{array}{l}36: \\
37: \\
38: \\
39: \\
40: \\
41:\end{array}$ & $\begin{array}{l}5.687-01 \\
1.929-03 \\
7.055-03 \\
4.230-03 \\
1.160-04 \\
1.143-01\end{array}$ & $\begin{array}{l}46: \\
47: \\
48: \\
49: \\
50: \\
51:\end{array}$ & $\begin{array}{l}57: \\
58: \\
59: \\
60: \\
61: \\
62:\end{array}$ & $\begin{array}{l}63: \\
64: \\
65: \\
66: \\
67: \\
68:\end{array}$ & $\begin{array}{l}\text { 69: } \\
70: \\
71: \\
72: \\
73: \\
74:\end{array}$ & $\begin{array}{l}\text { 75: } \\
76: \\
77: \\
78: \\
79: \\
80:\end{array}$ \\
\hline & "U 237 " & 31: & $2.450-05$ & & & 52: & & & & \\
\hline
\end{tabular}




\section{LEL 'NT \\ EHD 4 BO EITES}

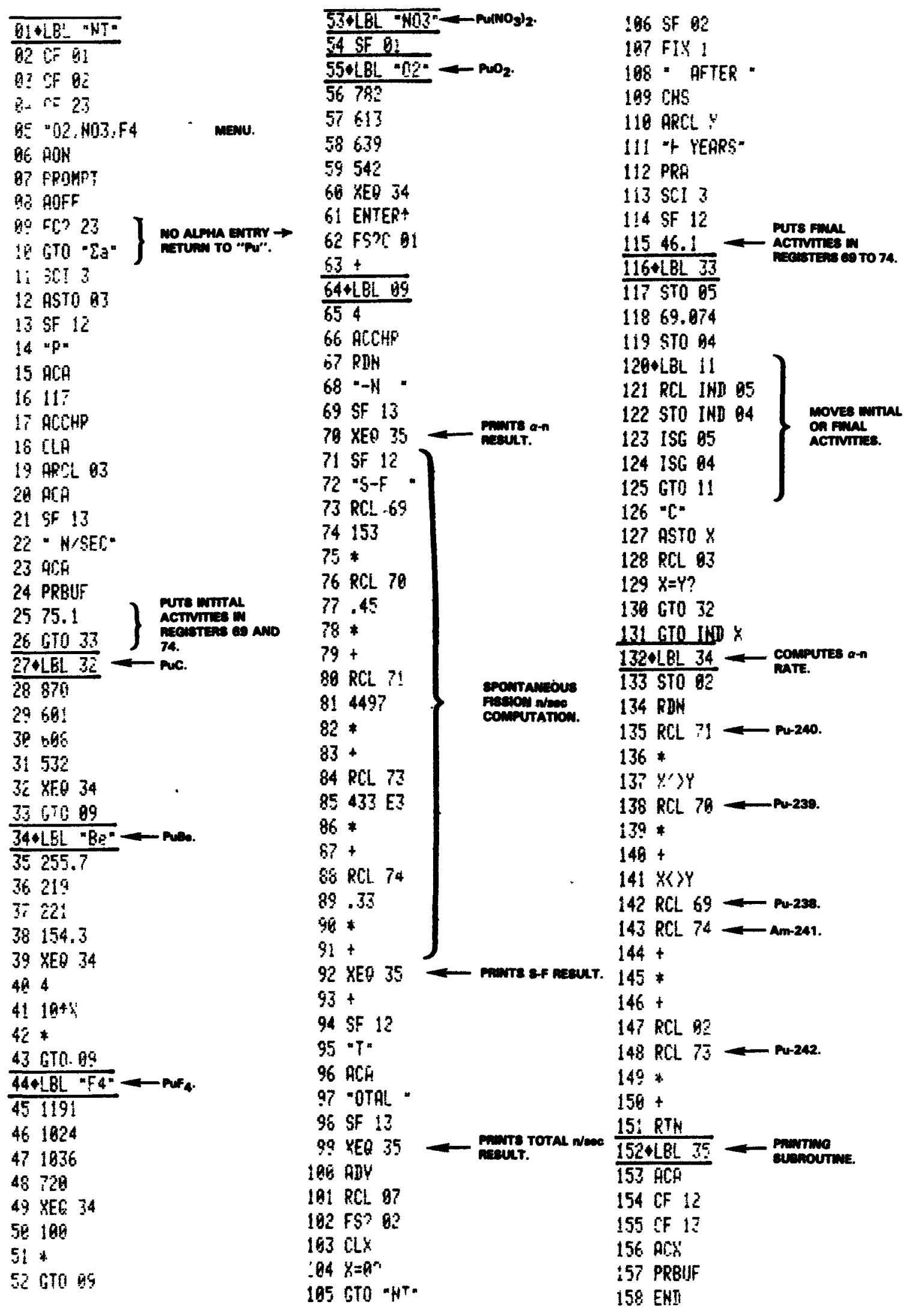


RHO-HS-ST-5 P

APPENDIX C

TIMEKEEPING PROGRAM

$c-1 / 2$ 


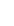




\section{USER INSTRUCTIONS}

1. XEQ SIZE 100; switch to USER mode. (Actually, the allowed memory size ranges from 021 to 215; using SIZE 100 is just a suggestion.) Read in the cards. The calculator must be in USER mode during card entry to read the USER mode assignments given to the first and third rows of keys.

2. XEQ "INITIAL" (or switch to USER mode and press shift STO). This subroutine zeroes the storage registers and then requests alphanumeric input for up to five names or identification numbers. Six characters are the maximum permitted in each. Press R/S after entering each name. If fewer than five names are needed, simply press R/S (before pressing any other key) when the next name is prompted for. The "* READY *" display indicates that all the names entered have been stored and the calculator is ready for use as a timekeeping tool.

2. Record each individual's dose rate, in $\mathrm{mR} / \mathrm{h}$ (or $\mathrm{mrad} / \mathrm{h}$ ) by using the number keys to enter the number and then pressing the $A, B, C, D$, or $E$ key in the top row. The calculator displays the most recent dose rate entry and the individual's accumulated dose in the following format.

$$
\text { dose rate, } m R / h(A, B, C, D \text {, or } E) \text { dose, } m R
$$

The number to the left is the current dose rate; the number to the right is the accumulated dose rate.

4. Once the dose rate is stored, the accumulated dose is not updated until a new dose rate is entered or the $A, B, C, D$, or $E$ key is pressed with no number entry. To facilitate these updates, the subrout ine "DVIEW" was created. Simply press shift XEQ and the calculator will automatically update and display each individual's dose. To remove the calculator from the repeating loop, simply press $R / S$.

5. When the work is complete, enter zero dose rate for each person.

6. Final doses may be obtained by pressing shift XEQ. The current dose rate should be zero. To obtain a final total listing by name and the total for the group, press shift RCL. If the printer is attached, the date and day of the week will also be printed. See the sample printout.

7. To facilitate stay time computations, there is a subroutine called "STATIM," which may be executed at any time by pressing shift SST. The calculator prompts for the dose limit; enter this and press R/S. Then the 41-CV prompts for the dose rate; enter this and press $R / S$. The calculator then computes the number of minutes it will take to reach the dose limit if the person stays in that dose rate. 
MEMORY USE:

\begin{tabular}{lll} 
00: & A \\
01: & B & Time \\
02: & C storage \\
03: & D & registers. \\
04: & E & \\
\hline 05: & A & \\
06: & B Dose rate \\
07: & C storage \\
08: & D registers. \\
09: & E &
\end{tabular}

10: A

11: B Accumulated

12: C dose storage

13: D registers.

14: $E$

15: A

16: B Alphanumeric

17: C name storage

18: D registers.

19: $E$

20: Total Dose

FLAG USE:

$F 05$ Set if no $A$.

$F 06$ Set if no $B$.

$F 07$ Set if no $C$

$F 08$ Set if no $D$.

$F 09$ Set if no $E$.

USUAL FINAL PRINTOUT:

FF!EPY $03 / 97 \cdot 1931$

FIHAL DOSES

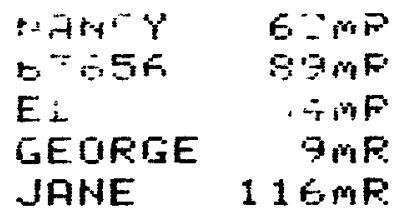

TOTAL $=311 \mathrm{mF}:$
SAMPLE CASE:

NAMES $E$ LET TES : HA
SUIE PALPH

FPl:it

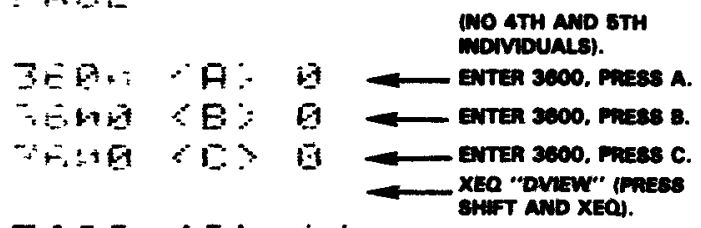

$3600<\bar{C}\rangle \quad 14$

$3690\langle A\rangle 13$

$3600<E \div \quad 12$

$36.00\langle A\rangle \quad 2$.

$3690\langle\mathrm{~B}\rangle$

$2600<0<22$

$3609<\mathrm{3} \div 33$

$3609<B>32$

$3600<c\rangle 31$

$3600\langle A\rangle 42$

$3600\langle B\rangle 41$

$3600\langle C\rangle \quad 41$

Tिब $2 B\rangle \in 1$

—XEO "DVIEW" AGAW.

BEAF $\langle A\rangle$ FI

$\rightarrow$ - $\angle B \% \quad-5$

$.1<0 \% \quad 89$

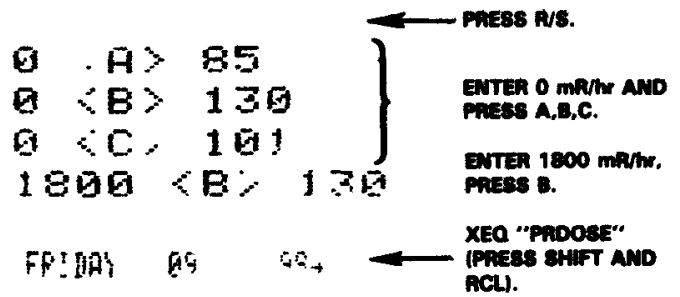

FIHAL DOSES

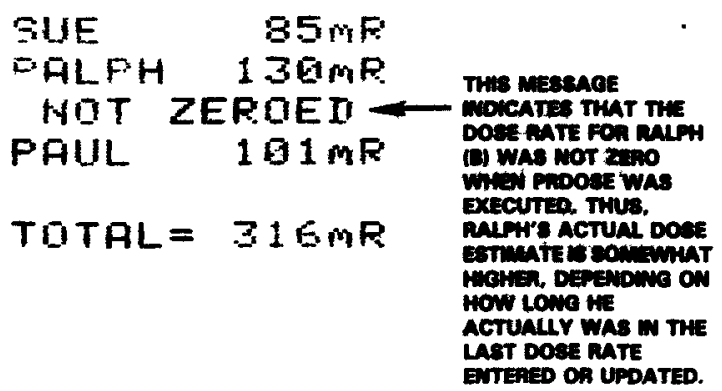


PROGRAM LISTING

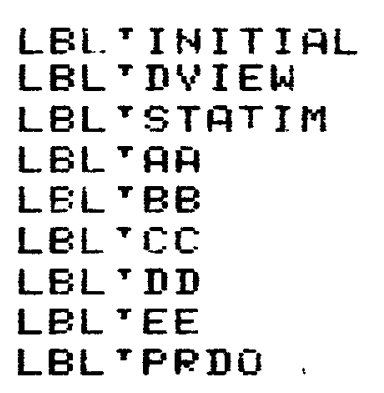

ENI TE BUIES

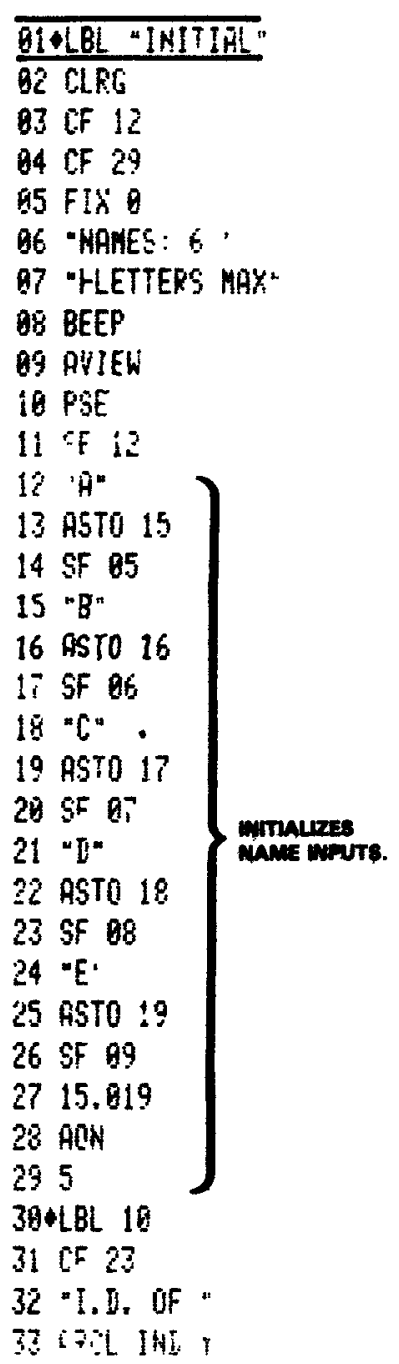

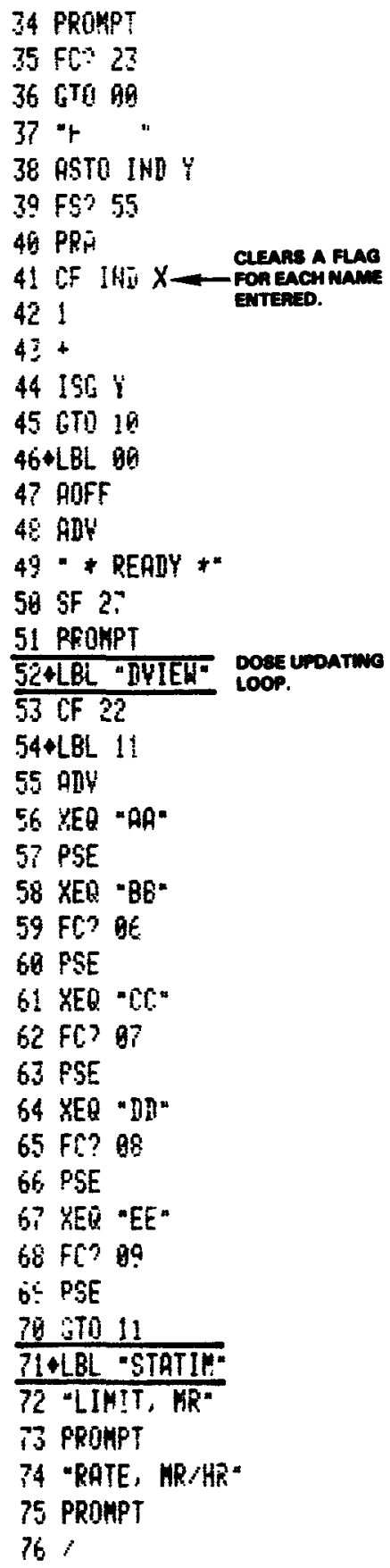

$\because 68$

$78 *$

7916

$80 x<Y$

$81 \mathrm{~K}=Y$ ?

$82 \mathrm{FIX} 1$

85 "S.T. = -

84 ARCL $X$

85 "F MIN"

86 FIX

8I AVIEK

98 RTN

$\overline{89+L B L \cdot A A^{\circ}}$

96 FC? 22

$91 \mathrm{RCL} 65$

92 FS? 85

93 RTN

94 CLA

95 ARCL $\mathrm{K}$

$96 Y 065$

$97 \mathrm{RCL} 80$

98 TIME

99 STO 69

$199 \mathrm{X}(3)$

101 HMS-

192 HR

$103 *$

$104 \mathrm{ST}+10$

105 "F $\langle A\rangle$ -

186 ARCL 10

167 AVIEN

108 RTN

$109+\angle B L{ }^{~} B B^{2}$

119 FC' 22

$111 \mathrm{RCL}$ B6

$112 \mathrm{FS}^{2}$ 6t

113 RTH

114 CLA

115 ARCL $\%$

$116 \mathrm{X}(1) 6$

$117 \mathrm{RCL}$ :

IIS TIME 
RHO-HS-ST-5 P

\section{PROGRAM LISTING}

\begin{tabular}{|c|c|c|c|}
\hline 119510 ด1 & $161 \mathrm{H}$ 5- & \multicolumn{2}{|l|}{29367009} \\
\hline $120 \mathrm{XYY}$ & $162 \mathrm{HR}$ & \multicolumn{2}{|l|}{$204+\mathrm{BL} \quad 02$} \\
\hline $121 \mathrm{HMS-}$ & $163 *$ & \multicolumn{2}{|l|}{265 - TUESDAY = } \\
\hline $122 \mathrm{HE}$ & $1645 T+13$ & \multicolumn{2}{|l|}{$286 \mathrm{GTO} 60$} \\
\hline $123 *$ & $165+t\langle D\rangle=$ & \multirow{2}{*}{\multicolumn{2}{|c|}{ 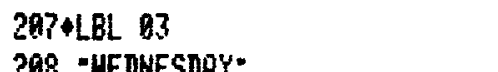 }} \\
\hline $1245 T+11$ & 166 ARCL 13 & & \\
\hline $125 " f\langle\theta\rangle "$ & 16? AYIEH & \multicolumn{2}{|l|}{20967006} \\
\hline $126 \mathrm{ARCL} 11$ & 168 RTN & \multicolumn{2}{|l|}{$2 ! 6+L B L \quad 64$} \\
\hline 127 AHIEN & $169+\angle B L{ }^{-E E^{n}}$ & \multirow{2}{*}{\multicolumn{2}{|c|}{211 "THURSDFY" }} \\
\hline 128 RTN & $\overline{170 \mathrm{FCO} 22}$ & & \\
\hline $129+\angle B L{ }^{\circ C C}$ & $171 \mathrm{RCL} 89$ & \multicolumn{2}{|l|}{$213 * L B L \quad 85$} \\
\hline $139 F C \times C 22$ & $172 F \$ ? 09$ & \multicolumn{2}{|l|}{$\begin{array}{l}214=\text { FRIBAY - } \\
215 \text { GTO GF }\end{array}$} \\
\hline $131 \mathrm{RCL} 67$ & $173 \mathrm{RTK}$ & 215 GTO 69 & \\
\hline 132 FS? $9 ?$ & $174 \mathrm{CLA}$ & $216+$ LBL. 86 & \\
\hline 133 RTN & 175 ARC $X$ & \multicolumn{2}{|l|}{217 - SATURDAY" } \\
\hline 134 CLO & $176 \times<>89$ & \multicolumn{2}{|l|}{$\begin{array}{l}218+L \text { LL } 90 \\
219=t\end{array}$} \\
\hline 135 ARCL $X$ & $177 \mathrm{RCL} 84$ & \multirow{2}{*}{\multicolumn{2}{|c|}{$\begin{array}{l}2 ! 9 " F " \\
220 \text { DATE }\end{array}$}} \\
\hline $136 x>97$ & 178 TIME & & \\
\hline 137 RC. 02 & 179 STO 84 & \multicolumn{2}{|l|}{221 ABATE } \\
\hline 138 TINE —-A THAE MODULE & $180 X\langle Y$ & \multicolumn{2}{|l|}{$\begin{array}{l}222 \text { PR. } \\
223 \text { SF } 12\end{array}$} \\
\hline 139 STO 82 & 181 HhS- & \multirow{2}{*}{\multicolumn{2}{|c|}{$\begin{array}{l}223 \text { SF } 12 \\
224 \text { GDY }\end{array}$}} \\
\hline $149 x<Y$ & $182 H$ & & \\
\hline 141 HNS- & $183 *$ & \multicolumn{2}{|l|}{225 "FINAL DOSES" } \\
\hline $142 \mathrm{HR}$ & $18457+14$ & \multirow{2}{*}{\multicolumn{2}{|c|}{$\frac{226 \text { PRA }}{227+\angle B L 19}$}} \\
\hline $147 *$ & $185 \mathrm{~F}\langle E\rangle=$ & & \\
\hline $144 S T+12$ & 186 ARCL 14 & \multicolumn{2}{|l|}{$228 C 1 X$} \\
\hline $145+\langle C\rangle$ & 187 AYIEH & \multicolumn{2}{|l|}{223 STO 20} \\
\hline $146 \mathrm{ARCL} 12$ & 188 RTH & \multicolumn{2}{|l|}{236 FIX 6} \\
\hline 147 AVIEN & 189+LBL "PRIOSF: & \multirow{2}{*}{\multicolumn{2}{|c|}{$\begin{array}{l}23115.919 \\
232 \text { ADV }\end{array}$}} \\
\hline $148 \mathrm{RTN}$ & 198 FC? 55 ) Exies oar & & \\
\hline$\frac{149 \cdot \angle B L=B D "}{100}$ & 191 GTO 19 J AND DATE & \multirow{5}{*}{\multicolumn{2}{|c|}{$\begin{array}{l}232 \text { ADY } \\
2335 \\
234 \div \text { LBL } 97 \\
235 \text { FS? IND X) LEAVE MANTOUT } \\
236 \text { C } 9 ! \\
237 \text { CLA }\end{array}$}} \\
\hline 150 FC?C 22 & $192 \mathrm{FIX} 6$ & & \\
\hline $151 \mathrm{RCL} 98$ & 193 of 12 & & \\
\hline $152 \mathrm{FS} ? 68$ & 194 ABY & & \\
\hline 153 RTN & 195 DATE & & \\
\hline 154 CLA & 196. $00 \mathrm{H}$ & \multicolumn{2}{|l|}{238 ARCL IND Y } \\
\hline 155 ARCL $X$ & $\$ 97610$ IND $X$ & \multicolumn{2}{|l|}{$\begin{array}{l}2395 \\
249+\end{array}$} \\
\hline $156 \times 1>08$ & 198+LBL & $240+$ & \\
\hline 157 RCL 93 & 199 " SUNDAY " & 241 RCL IHI $\mathrm{X}$ & \\
\hline 158 TIME & $280 \mathrm{GTO} 60$ & $2425 T+20$ & \\
\hline 159 sTo 83 & $201+\angle B L$ 6! & 243 XEO 2 & \\
\hline $160 X\langle Y Y$ & 262 - BONDA1 " & 244 RDN & \\
\hline
\end{tabular}




\section{RHO-HS-ST-5P}

\section{PROGRAM LISTING}

2455

246 -

247 RO INI Y

248 - NOT ZEROEE

$249 x+0^{\circ}$

250 AYIEH

251 RDN

2521

$253+$

$254156 y$

255 6T0 Q?

256*LBL O1

257 ADY

258 -TOTHL $==$

259 RCL 20

260 XEQ Q2

261 ADY

$262 \mathrm{ABV}$

263 ADV

264 ADV

265 ADY

266 RTH

2674LBL B?

$268 \mathrm{FC}>55$

26961069

270 ACA

271 CLA

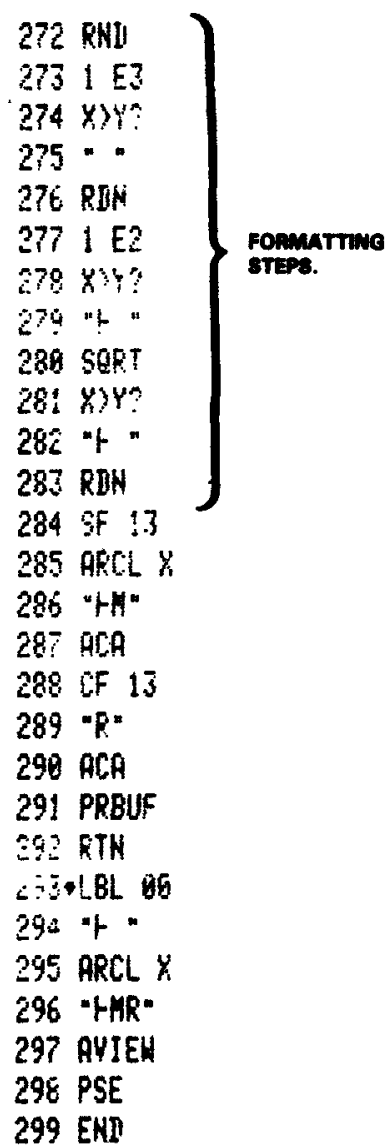

$273+53$

$274 \times) Y$ ?

275 *

$276 \mathrm{REN}$

$277 \perp E 2$

279 Y)?

274 "f "

289 SQRT

$281 \mathrm{X}) \mathrm{Y}$ ?

286 "f

283 RBN

285 ARCL $X$

286 "H"

$287 \mathrm{ACA}$

288 of 13

289 " $\mathrm{R}$ "

290 ACA

291 PRBUF

$292 \mathrm{RTH}$

295 ARCL $X$

296 "FMR

298 PSE

299 ENI $\therefore$ 든 
RHO-HS-ST-5 P

Condensed, but Slower Version of Timekeeping Program.

\section{LBL' INITIAL \\ LBL' DVIEW \\ LBL'STAT IM \\ LBL TPRDOSE \\ ENI 634 EVIES}

Q1+LB $B_{L}$ "INITIFE"

B2 CLPG

$63 \mathrm{CF} 12$

04 CF 25

Q5 FIX:

Q6 "NAMES 6'

67 "HETTERS MAK"

Q8 BEEP

B9 AYIEN

16 PSE

11 SF 12

12 " $\beta$ "

13 ASTO 15

14 SF 95

15 " $\mathrm{B}$ "

16 ASTO 16

17 SF 96

18 " $\mathrm{C}$ "

19 ASTO 17

20 SF 97

21 " $\mathrm{D}$ "

22 ASTO 18

27 SF 68

24 "E"

25 ASTO 19

26 SF 09

3715.019

28 AON

295

$3 \overline{38+L B L \quad 18}$

31 CF 23

32 "I. D. OF *

33 ARCL IND Y

34 PROMPT

$35 \mathrm{FC} 23$

$36 \mathrm{GTO}$ ด8

37 r

38 ASTO INI $Y$

$39 \mathrm{FS} 55$

48 PRA

41 CF iNI

421

$43+$

$44150 r$
45 GTO li

$464 \mathrm{LB}_{1}$.

47 AOFF

4 ADV

49 " * READY *"

50 SF 27

51 PROHPT

520LBL "DYIEW"

53 cf 22

$54+$ LEL 11

55 ADV

Sf YEO A

57 PSE

58 XEQ

$59 \mathrm{FC}$ ?

60 PSE

61 XEO C

$62 \mathrm{FC} 27$

63 PSE

64 XEQ D

$65 \mathrm{FC} 798$

66 PSE

Q7 XEQ E

$68 \mathrm{FC}$ ? 99

69 PSE

70 GTO 11

71+LBL "ETATIT"

72 "LIMIT, MR"

73 PROAFT

74 "RATE, ME/HP"

75 PROHPT

$76 \%$

7760

$73 *$

7910

$80 \% Y$

S! $Y\{=Y$ ?

$82 \mathrm{FIX}:$

$87 \cdot 5.1 .=\cdot$

84 ARCL

$85^{\circ}$ 'F MIN'

St FIY

87 PUIEK

38 RTN

$89+\angle B_{L} 20$

$90 ; 322$

$91 \% \geqslant i$

92 F[? 2

93 RCL IND $x$

$94 \mathrm{FS}$ IHD $Y$
95 RTN

96 ASTO L

97 CLA

98 ARCL $Y$

99 ARCL :

100 X() IND Y

1815

102 ST- 2

163 RDN

164 RCL IND Y

185 TIME

106 STO IND T

$187 \mathrm{XYY}$

108 HiS-

$109 \mathrm{HR}$

$110 *$

11119

$112 \mathrm{sT}+\mathrm{Z}$

113 RDN

114 ST+ INB Y

115 ARCL IND Y

I16 AYIEY

117 RTN

118*LBL

- 1195

$120 \cdot\langle\mathrm{A}\rangle=$

121 GTO 20

$122+\angle B L B$

1236

$124 *\langle 8\rangle \cdot$

125 GTO 29

I26+LBL C

1277

128 - $\{C\}$.

129 GTO 28

1350LBL I

1318

132 * $\langle$ D $\rangle=$

133 GTO 28

134+LBLE

1359

136 " $(E) "$

137 GTO 29

1384LBL "PRDOSE:

$139 \mathrm{FC}^{7} 55$

14 CTO 19

141 FIX 6

142 CF 12

143 ADV

144 JATE 
Condensed, but Slower Version of Timekeeping Program.

\begin{tabular}{|c|c|c|}
\hline 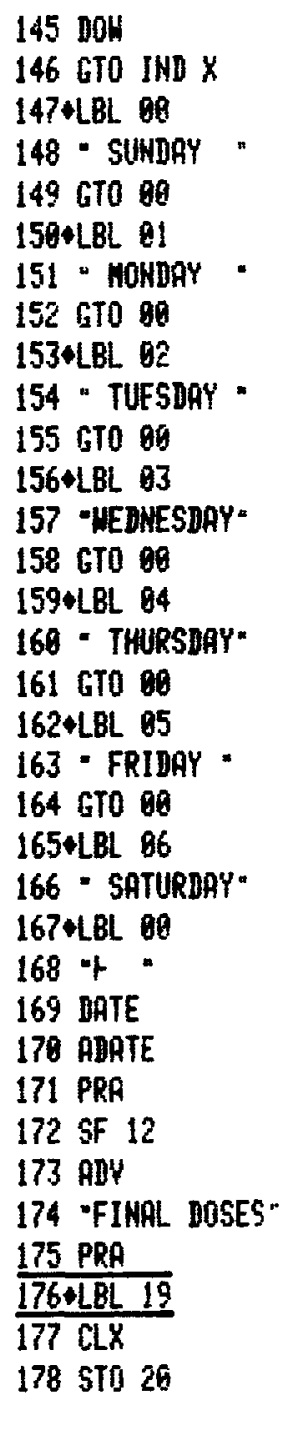 & 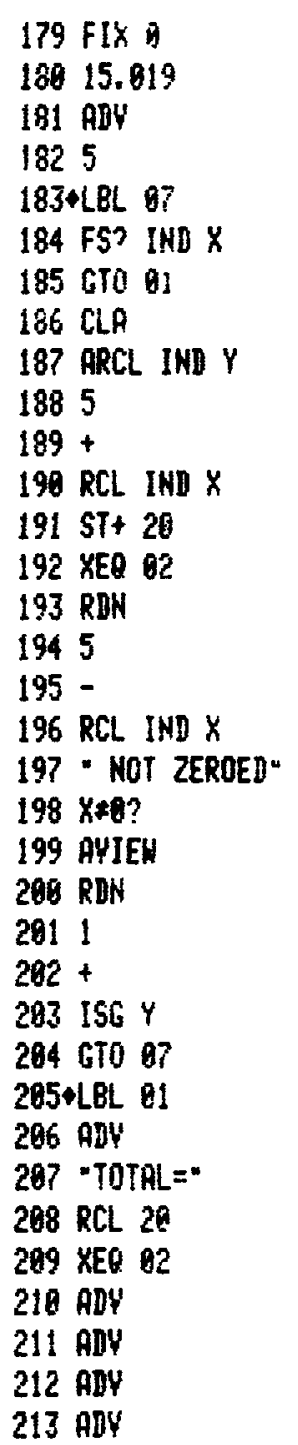 & 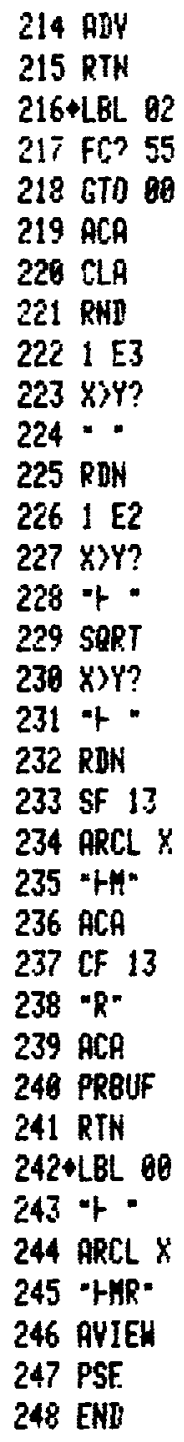 \\
\hline
\end{tabular}




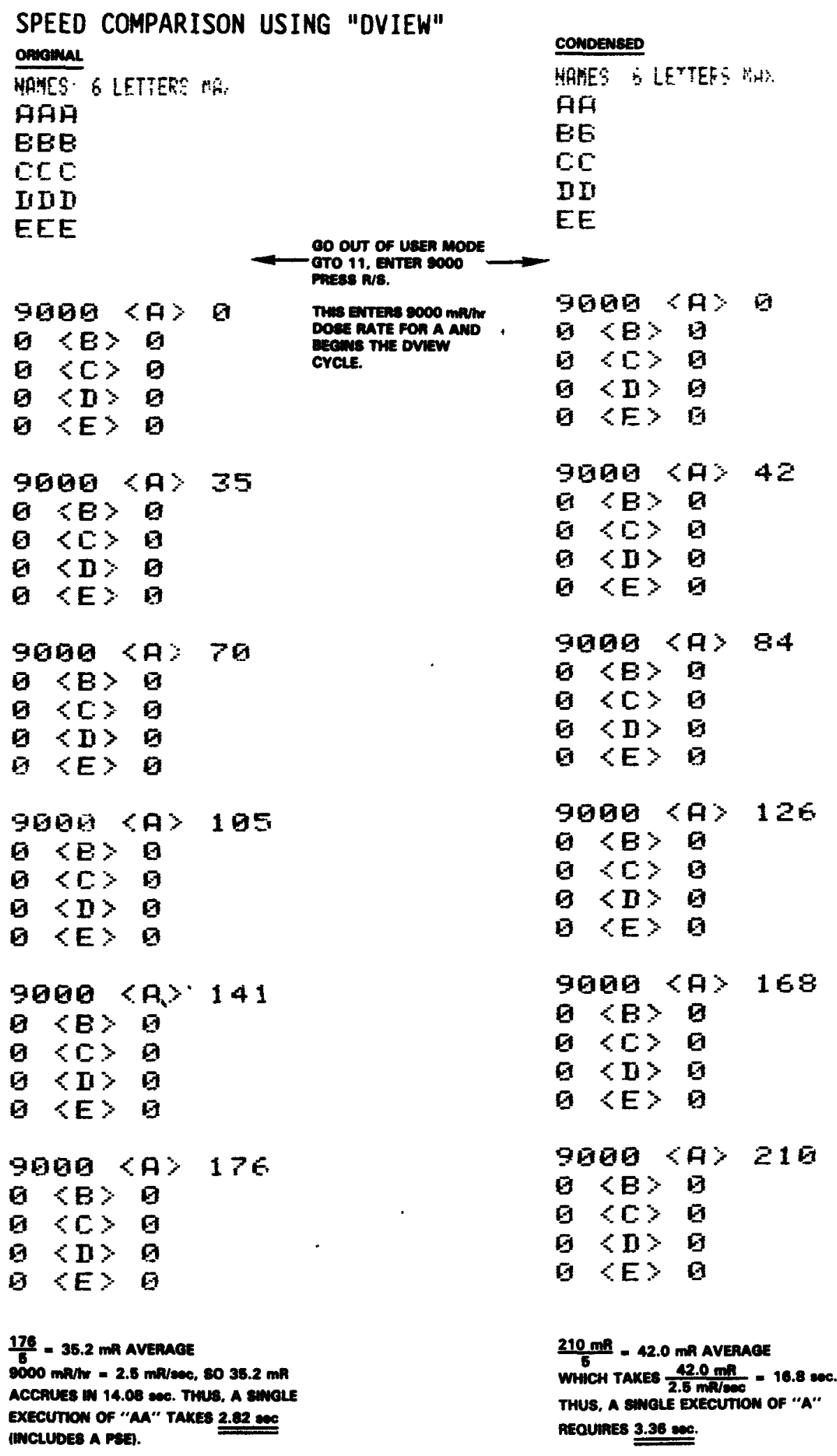

DUFERENCE 0.84 sec. 
RHO-HS-ST-5 P

APPENDIX D

EMERGENCY RESPONSE - "H/Q"

$0-1 / 2$ 


\section{USER INSTRUCTIONS}

\section{A. Enter program (read three cards).}

B. Press XEQ "H/Q." Program may be run with or without a printer. If the printer is off or not attached, all displayed results will stop program execution. To continue, simply press R/S.

C. Input requested information; press $R / S$ after each entry.

1. Release height $(h)$ in meters.

2. Wind speed at the point of release $(u)$ in meters per second.

3. Downwind distance to receptor $(x)$ in meters.

4. If sector averaging (22.5 degree sectors) is desired, enter any number. For centerline $X / Q$, do not enter any number.

5. Stability class - enter one of the following:

VS $=$ Hanford very stable

MS = Hanford moderately stable

UN = Sutton's unstable

$N=$ Sutton's neutral

6. Specific meterological parameters are entered last. Tables of recommended values are given in Tables 3 and 4. Stable conditions require a wind meander parameter; neutral and unstable conditions require input of Sutton's parameters.

NOTE: The wind meander is used by only the horizontal dispersion variables $\left(\sigma_{y}\right)$. Thus, when sector averaging, wind meander is not prompted.

D. Once $X / Q$ has been computed, the program asks "INPUT LIST?" which means the option to review input parameters and computed values of $\sigma_{y}$ and the vertical dispersion variable $\left(\sigma_{Z}\right)$ is available. If a listing is desired, enter any number and press R/S. Press R/S to view successive parameters if no printer is attached or if the printer is off. If the listing is not desired, enter nothing; press $R / S$.

NOTE: When sector averaging, the parameter $\sigma_{y}$ is replaced with the quantity $(x / 8) \sqrt{\pi / 2}$. 
E. The next option facilitates dose computation for each isotope using unit dose factors and curies released.

- If such $a_{3}$ computation will be done, enter the first dose factor $\left(\mathrm{rem} / \mathrm{Ci} . \mathrm{m}^{3} / \mathrm{s}\right)$ and the activity released for each isotope. The program computes the dose from each isotope and displays it. Once all isotopes have been computed, the program prompts for another dose factor. Press $R / S$ and the sum total will be displayed.

- If no dose computation is desired, enter nothing; press R/S and "H/Q" will execute from the beginning.

F. Finally, the user may simply want to change one or two input parameters and rerun the $X / Q$ calculation. This capability is realized in two ways:

1. Switch to USER mode.

a. To change $h$, enter the new $h$ and press $A$.

b. To change $u$, enter the new $u$ and press $B$.

c. To change $x$, enter the new $x$ and press $C$.

d. To switch to sector averaging or to remove this option, press $\mathrm{H}$.

e. To change stability class, press $D$. The MET choices will then be displayed. Enter the new MET and press R/S. The program will store and execute the new MET, prompting for the necessary parameters. If no change in these parameters is desired, enter nothing; press R/S. Program "H/Q" automatically begins execution.

f. To execute the program, press $E$.

2. Execute "H/Q" from the beginning.

a. As each item is prompted, enter either the new value and press $R / S$ or the previous value by pressing only $R / S$.

b. Previous values of $h, u$, and $x$ are recalled to the $x$-register just prior to their prompt and may be viewed by clearing the display. Centerline $X / Q$ will be computed unless sector averaging is requested.

G. Mathematical formulas are listed in the " $X / Q$ " program instructions. 
VALUES

TABLE 3. Values for Wind Meander $\left(\sigma_{\theta} u\right)$.

\begin{tabular}{c|c|c|c|c}
\hline \multirow{2}{*}{$\begin{array}{l}\text { Release } \\
\text { duration }\end{array}$} & \multicolumn{4}{|c}{ Wind Speed } \\
\cline { 2 - 5 } & $1 \mathrm{~m} / \mathrm{s}$ & $2.5 \mathrm{~m} / \mathrm{s}$ & $5 \mathrm{~m} / \mathrm{s}$ & $10 \mathrm{~m} / \mathrm{s}$ \\
\hline $10 \mathrm{~min}$ & .024 & .10 & .20 & .30 \\
$60 \mathrm{~min}$ & .04 & .15 & .25 & .35 \\
$120 \mathrm{~min}$ & .06 & .25 & .35 & .45 \\
$240 \mathrm{~min}$ & .10 & .40 & .50 & .60 \\
$480 \mathrm{~min}$ & .18 & .60 & .70 & .90 \\
\hline
\end{tabular}

TABLE 4. Values for Sutton's Parameters, $C_{y}$ and $C_{z}$.

\begin{tabular}{|c|c|c|c|c|}
\hline Release level & Wind speed & $C_{y}, C_{z}$ & $\begin{array}{l}n=.20 \\
\text { Unstable }\end{array}$ & $\begin{array}{l}\mathrm{n}=.25 \\
\text { Neutral }\end{array}$ \\
\hline \multirow{3}{*}{ Ground } & $1 \mathrm{~m} / \mathrm{s}$ & $\begin{array}{l}c_{y} \\
c_{z}\end{array}$ & $\begin{array}{r}.35 \\
.35\end{array}$ & $\begin{array}{r}.21 \\
.17\end{array}$ \\
\hline & $5 \mathrm{~m} / \mathrm{s}$ & $\begin{array}{l}c_{y} \\
c_{z}\end{array}$ & $\begin{array}{l}.30 \\
.30\end{array}$ & $\begin{array}{r}.15 \\
.14\end{array}$ \\
\hline & $10 \mathrm{~m} / \mathrm{s}$ & $\begin{array}{l}c_{y} \\
c_{z}\end{array}$ & $\begin{array}{l}.28 \\
.28\end{array}$ & $\begin{array}{r}.14 \\
.13\end{array}$ \\
\hline Elevated & $\begin{array}{c}1 \mathrm{~m} / \mathrm{s} \\
5 \mathrm{~m} / \mathrm{s} \\
10 \mathrm{~m} / \mathrm{s}\end{array}$ & $\begin{array}{l}c_{y}, c_{z} \\
c_{y}, c_{z} \\
c_{y}, c_{z}\end{array}$ & $\begin{array}{r}.30 \\
.26 \\
.24\end{array}$ & $\begin{array}{r}.15 \\
.12 \\
.11\end{array}$ \\
\hline
\end{tabular}




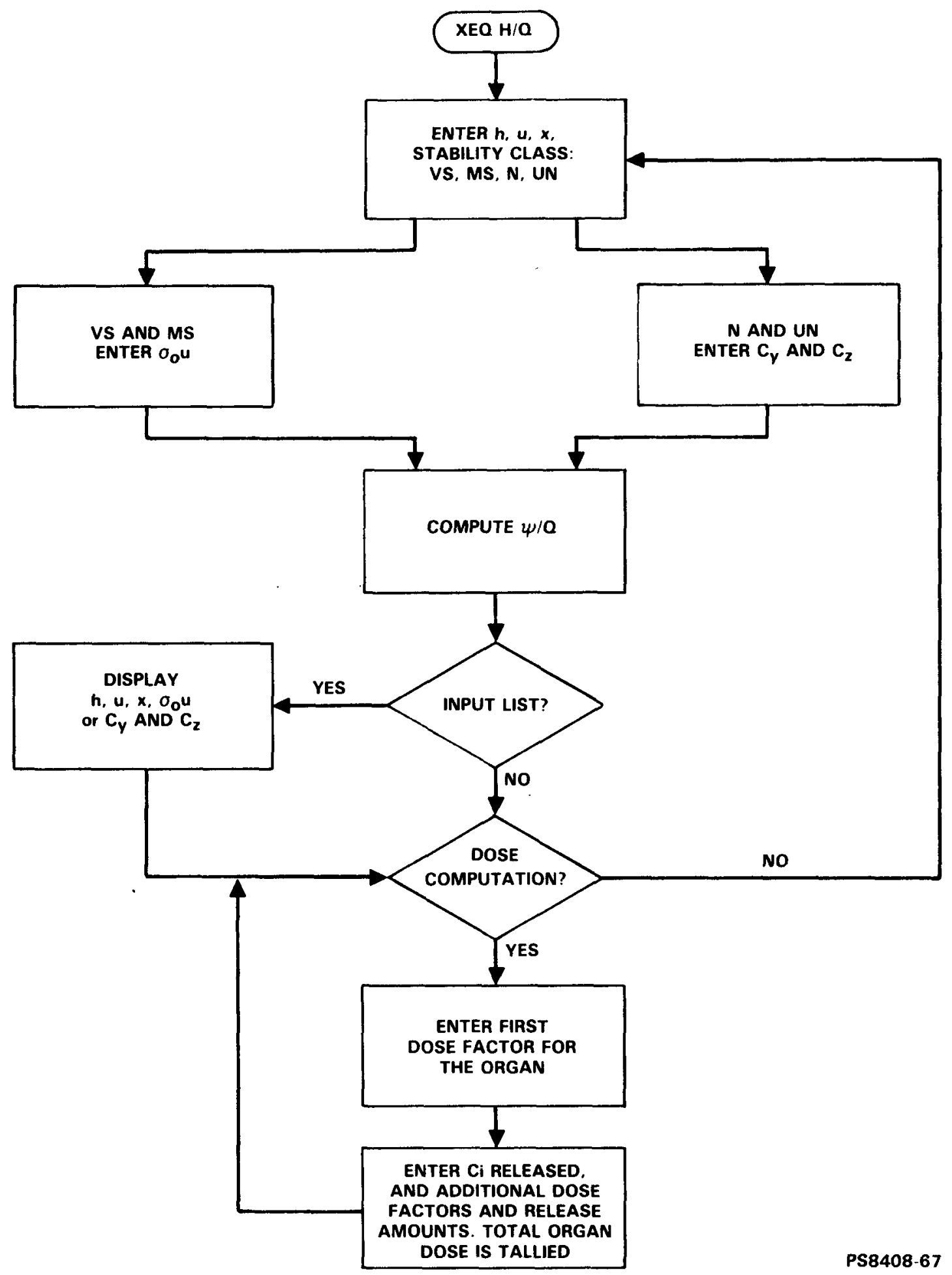

FIGURE 3. Flowsheet for Program "HQ." 


\section{PROGRAM LISTING}

$$
\begin{aligned}
& \text { LEL'H } \\
& \text { ENI } 65 \text { E EYTEC }
\end{aligned}
$$

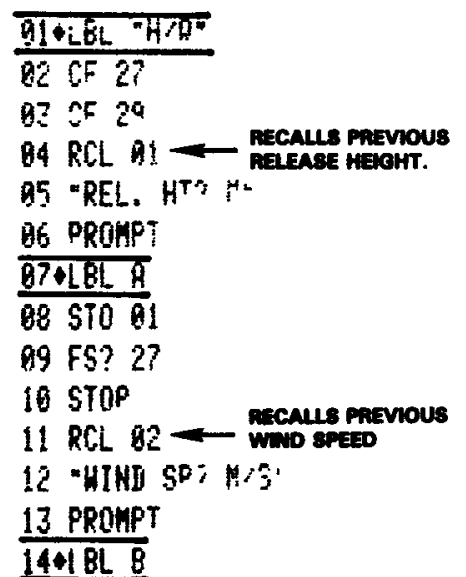

$\frac{14+18 L}{155700}$

$16 \mathrm{PCL}$ Gi - RECALLS $\mathrm{x}$.

17 FS? 27 COMPUTES TIME OF

18 GTO C FLOHT IN USER.

19 "DISTANCE"

29 PROMPT

$\left.\begin{array}{l}\frac{210 L B L C}{22} 5 T 093 \\ 25 \mathrm{RCL} 82 \\ 24,\end{array}\right\} t=x / 0$

25 STC 94

$26 \mathrm{FS}^{27}$

27 STOP

28 CF 9

29 CF 22

30 -SECTOR AHE

31 PROMPT

$32 \mathrm{FC} 222$

33 GTO D

$34 *$ LBL

$35 \mathrm{FCN}$ OR ) CLEARS FLAO OIF 36 SF A9

37 FS? 27

30 STOP

$390 \mathrm{LBL}$

4! OF 2?

41 "MET' US, MS, W. BN.

$42 \mathrm{AON}$

43 PROMF"

44 AOFF

$45 F S^{2} 23$

46 ASTO OA

$47 \mathrm{SF} \mathrm{Q}_{2}$
$\frac{48+L B L E}{49 C F \quad 9 !}$

$50 \mathrm{FC}^{2} 5$

51 CF $2 ;$

52 GTO IND OG

53+LBL -IIN"

54 FC?

55 GTO म1

56.

57 5TO 09

$58 \mathrm{RCL}$ B?

59 "CY, $C Z$ ?"

60 PROMPT

61 STO 07

6251088

63 GTO OI

$64+\mathrm{LBL}-\mathrm{H}^{*}$

55 FC 02

6607001

67.875

68 STO 99

$69 \mathrm{FS} ?$

70 GTO 98

71 RCL 67

72 "CY $\%$

73 PROHPT

$745 T 0 \quad 07$

$75+\angle B L \quad 66$

$76 \mathrm{RCL}$ 68

77 - $\mathrm{CZ}>$

78 PROMPT

7951006

BQ+LBL G!

$81 \mathrm{RCL} G \mathrm{E}$

उĉ RCL $8 \overline{3}$

83 RCL 89

$84 \mathrm{YHX}$

852

8E SQRT

87 ;

89 *

$892 \mathrm{E}$

$99 x>4=$

$91 \mathrm{xizy}$

9251095

93 LASTY

$94 \mathrm{KCL} 87$

95 *

96 SF 01

पT GT 22

(1)

EMSURES PaOcRaM

aN AVEEw.

avew.

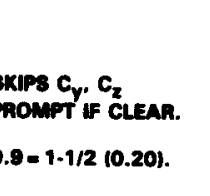

SKIP3 C. C

$0.8=1.1 / 2(0.20)$

(n)

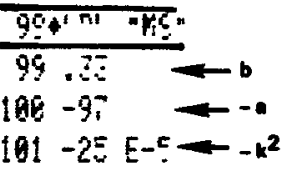

103 GTO af

163+LB: AY?:

10449

$1851, x=0$

$186-34 \leq-$.

$107-88 \mathrm{E}-\mathrm{c} \div-\mathrm{k}^{2}$

$\overline{192+\mathrm{LB}_{\mathrm{L}}^{\prime} \mathrm{OP}}$

$\longdiv { 1 6 9 \mathrm { RCL } }$

$116 \times 12$

$111 *$

$112 \mathrm{E}+\mathrm{X}-1$

$113 *$

$114 x(Y Y\}$ v' and Ms

115 RCL 84

SKIPS $C_{y}$ AND $C_{z}$ PMOMPTS If CLEAR.

$116 *$

$117+$

118 SQRT

119 STO 95

120 FS? 80 ) SXIPS Oy WHEN

121 GTO 22. SECTOA

122 FC?C 92 sxpes 10 ul monpt

123 GTO ge UHen ciens.

124 CF 22

125 -SIGTHETA*1 ?"

126 PROHPT

$127 \mathrm{FC} 22$

128 GT0 89

12957067

138232

$13 ! *$

13213

$133+$

134510 88

$135 \mathrm{RCL}$.

$136 x+2$

1372

$138 *$

139 ,

149 \$T0 99 


\section{PROGRAM LISTING}

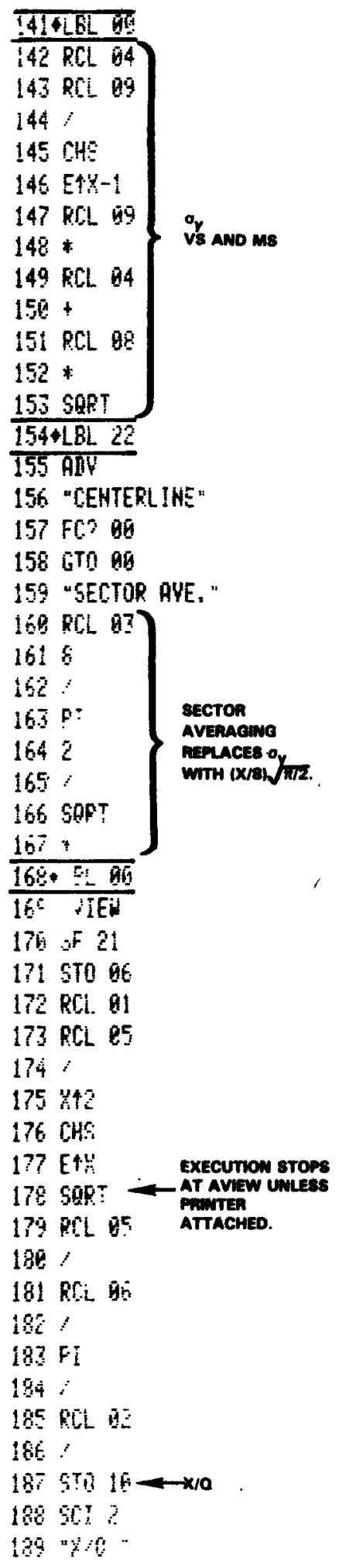

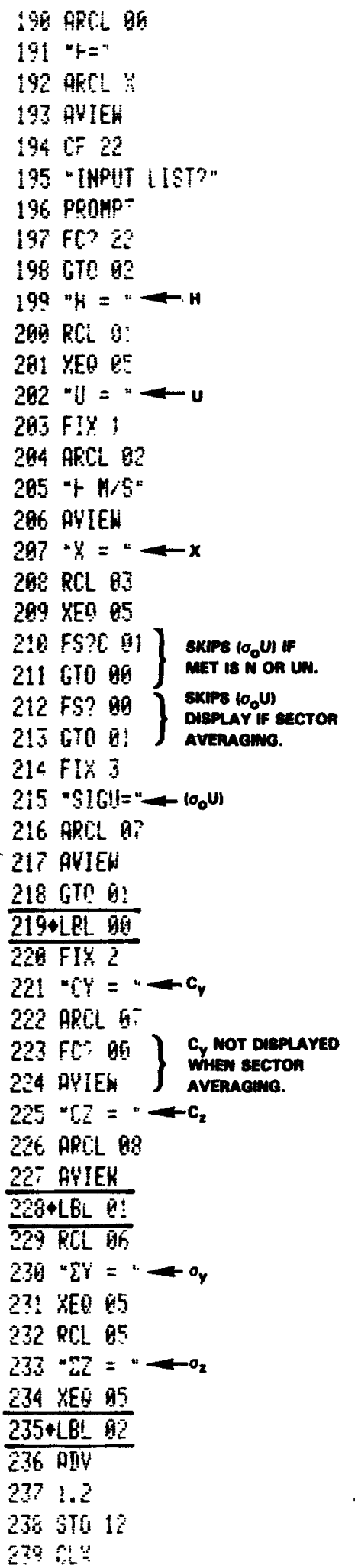

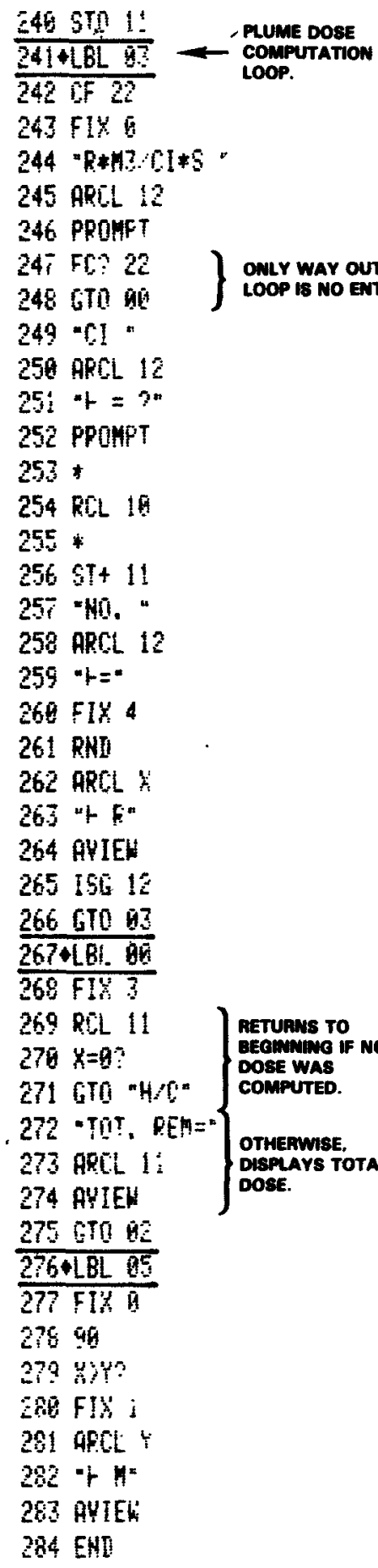

246 STI I:

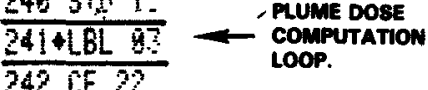
$247 \mathrm{FIH}$ 244 "R+HI:CI\$S" 245 AREL 12 246 PQOAFT $24 \mathrm{FC}: 22$ 248 GTO $249-\mathrm{CI}$. 250 ARCL 12 $25:$ " $=2$ " 252 PPONPT 253 * $254 \mathrm{RCL} 1 \mathrm{~A}$ $255 *$ 256. $5 T+11$ $25 \%$ HI. . 250 ARCL 12 259 " $k=$ $269 \div 1 X 4$ 261 RNI 262 ARCL $\%$ 263 "F" 264 AVIEH 265 IS6 IF 266 G70 93 267+LBL. 89 $268 \mathrm{FIE}^{3}$ $269 \mathrm{ROL} 11$ $270 x=9$ ? 271 GTO "HA: 272 "TIT, $\mathrm{DE} M=$ 273 ARCL I: 274 AVIEH 275 CTO 2764LBL 95 277 FI\% 27845 279 और? ERE FIA 291 AfE $202+7=$ 263 PUIEL $2 \mathrm{R} 4 \mathrm{EHI}$ 
MEMORY USE:

00: Stability class

01: Release height (m)

02: Wind speed $(\mathrm{m} / \mathrm{s})$

03: Downwind distance (m)

04: Time of flight (s)

05: $\sigma_{z}$

06: $\sigma_{y}$

07: $\left(\sigma_{\theta} u\right)$ or $C_{y}$

08: $A$ or $C_{7}$

09: $\alpha$ or $(1-n / 2)$

10: $\psi / Q$

11: Dose sum, rem

12: Pointer
FLAG USE :

00: Sector averaging

01: Met is $N$ or UN

02: Whenever label $D$ is run 
RHO-HS-ST-5 P

APPENDIX E

"X/Q - HANFORD"

$E-1 / 2$ 


\section{USER INSTRUCTIONS}

A. Attach printer and partition calculator memory as follows before reading in the program ( 7 cards): SIZE $=23+3 \mathrm{~N}$, where $\mathrm{N}$ is the number of downwind distances to be used. In the standard HP-4ICV, the maximum SIZE allowed with "X/Q" is 096, which allows 24 distances.

B. Execute "X/Q" and enter the following necessary information, pressing R/S after each input.

1. Release height $(h)$ in meters.

2. Wind speed $(u)$ in meters per second.

3. Deposition speed $\left(v_{d}\right)$ in centimeters per second. Zero is automatically entered if only $R / S$ is pressed.

4. Building area (S) in square meters. If building wake effects will not be included, simply press $R / S$ without entering a number. Zero area is automatically entered.

5. If sector averaging is desired, enter any number. To leave out the sector average option, enter nothing; press $R / S$.

6. The first distance to be used. The program then prompts for each distance. If sector averaging is selected, the program also prompts for the population at each distance. Please bear in mind the following:

a. To reduce program running time, enter distances in increasing order.

b. Zero is not an allowed distance.

c. Unit populations are automatically entered if no other values are input by the user.

d. Distance (and population) data are not altered by program execution. If data from the previous run (or data card entry) will be used, press R/S when the first distance prompt appears.

When all distances have been entered, press $R / S$ at the next distance prompt. 
7. Hanford atmospheric stability class. Because the calculator is now in ALPHA mode, simply enter the appropriate letters, as follows:

$$
\begin{aligned}
& \text { VS - Hanford very stable } \\
& \text { MS - Hanford moderately stable } \\
& N \text { - Sutton's neutral } \\
& U N \text { - Sutton's stable. }
\end{aligned}
$$

If all four stability classes will be run, enter nothing; just press $R / S$.

8. Specific meterological parameters (1isted here) are entered last. Recommended values are given in Tables 5 and 6.

VS \& MS: Enter value of wind meander $\left(\sigma_{\theta} u\right)$ or the release duration if wind speed is $1 \mathrm{~m} / \mathrm{s}$.

$N$ \& UN: Enter value of $C_{y}$ and $C_{z}$.

If all four classes will be run, the program prompts for all the necessary parameters before beginning $X / Q$ computations.

C. Once the output is complete, the input data may be changed and the program run again. To restart from the beginning, press $R / S$. To change only a few items and execute, switch to USER mode and input the changes using local alpha labels as follows:

1. To change $h$, enter the new $h$ and press $A$.

2. To change $u$, enter the new $u$ and press $B$.

3. To change $v_{d}$, enter the new $v_{d}$ and press $F$.

4. To change $S$, enter the new $S$ and press $G$.

5. To switch to sector-averaged $X / Q$ or to remove this option, press $H$. Be sure to enter population data, if necessary.

6. To change distances (and populations if sector averaging), press C and enter the requested information.

7. To change stability class or stability class parameters, press 0. The program begins executing with the "MET? VS, MS, N, UN" prompt. Enter your selections according to the instructions given in the preceding steps B.7. and B.8. Please bear in mind the following.

a. The wind meander parameter required by VS and MS classes is stored so that it never needs to be reentered unless it changes. When it or release duration is prompted for, press $R / S$ and no changes will be made. 
b. Sutton's parameters are also stored and only need to be reentered when changing from all four classes to a specific class (or vice versa).

8. To execute a run without changing stability class, press $E$. 
VALUES

TABLE 5. Values for Wind Meander $\left(\sigma_{\theta} u\right)$.

\begin{tabular}{l|l|l|c|c}
\hline \multirow{2}{*}{$\begin{array}{l}\text { Release } \\
\text { duration }\end{array}$} & \multicolumn{4}{|c}{ Wind speeds } \\
\cline { 2 - 5 } & $1 \mathrm{~m} / \mathrm{s}$ & $2.5 \mathrm{~m} / \mathrm{s}$ & $5 \mathrm{~m} / \mathrm{s}$ & $10 \mathrm{~m} / \mathrm{s}$ \\
\hline $10 \mathrm{~min}$ & .024 & .10 & .20 & .30 \\
$60 \mathrm{~min}$ & .04 & .15 & .25 & .35 \\
$120 \mathrm{~min}$ & .06 & .25 & .35 & .45 \\
$240 \mathrm{~min}$ & .10 & .40 & .50 & .60 \\
$480 \mathrm{~min}$ & .18 & .60 & .70 & .90 \\
\hline
\end{tabular}

TABLE 6. Values for Sutton's parameters, $C_{y}$ and $C_{z}$.

\begin{tabular}{l|c|c|c|c}
\hline Release level & Wind speed & $c_{y}, c_{z}$ & $\begin{array}{c}n=.20 \\
\text { Unstable }\end{array}$ & $\begin{array}{c}n=.25 \\
\text { Neutral }\end{array}$ \\
\hline \multirow{4}{*}{ Ground } & $1 \mathrm{~m} / \mathrm{s}$ & $c_{y}$ & .35 & .21 \\
& $c_{z}$ & .35 & .17 \\
\cline { 2 - 5 } & $5 \mathrm{~m} / \mathrm{s}$ & $C_{y}$ & .30 & .15 \\
& & $C_{z}$ & .30 & .14 \\
\cline { 2 - 5 } & $10 \mathrm{~m} / \mathrm{s}$ & $C_{y}$ & .28 & .14 \\
& & $C_{z}$ & .28 & .13 \\
\hline \multirow{3}{*}{ Elevated } & $1 \mathrm{~m} / \mathrm{s}$ & $C_{y}, C_{z}$ & .30 & .15 \\
& $5 \mathrm{~m} / \mathrm{s}$ & $C_{y}, C_{z}$ & .26 & .12 \\
& $10 \mathrm{~m} / \mathrm{s}$ & $c_{y}, c_{z}$ & .24 & .11 \\
\hline
\end{tabular}




\section{SAMPLE OUTPUT}

The sample on the left was produced using the sector-averaging option. Only one atmospheric stability class was chosen, Hanford moderately stable with a release duration of 1 .

The sample on the right was generated by clearing the sector-averaging option and choosing all four classes.

$$
\begin{array}{r}
\text { *** } * \text { X } \\
\text { REY } 4 \text { **** } \\
3-13-94
\end{array}
$$

RELERSE HEIGHT. Q.A

UIND SPEED $1.0 \mathrm{~m}$ इQC

DEP. SPEEU: 1.190 in'se?

BUILDING AREA. $40 Q$ SA

* SECTOR AYERAGED $x / \theta *$

Distance populftion

$8000 \quad i$

$15500 \quad 2089$

TOTRL 2091

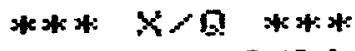

$$
\begin{aligned}
& \text { REV } 4 \text { 3-13-84 }
\end{aligned}
$$

RELEASE HEIIHT $\cdot 0 . \bar{E}$

HIND SPEED. 1.9 " $S E D$

DEP. SPEEI: G. 100 UN'SEO

BUILDING AREA: $\$ \bar{H}$ S 39 A

o日*u. 0.040 n $\sec$

VER' STABLE $Y \quad X>Q$ Fo

$8060 \quad 4.25-95 \quad 6.619$

$155801.45-85 \quad 8.45 \%$

\begin{tabular}{|c|c|c|}
\hline & EUTR & $=0.17$ \\
\hline $\begin{array}{c}x \\
8990 \\
15590\end{array}$ & $\begin{array}{l}X>Q \\
2.39-46 \\
7.42-6\end{array}$ & $\begin{array}{l}\text { Fd } \\
\text { Q.988 } \\
0.896\end{array}$ \\
\hline & YSTA & \\
\hline $\begin{array}{c}x \\
8980 \\
15590\end{array}$ & $\begin{array}{l}x<0 \\
4.69-67 \\
1.42-6\end{array}$ & $\begin{array}{l}F d \\
0.950 \\
0.953\end{array}$ \\
\hline
\end{tabular}

$-$

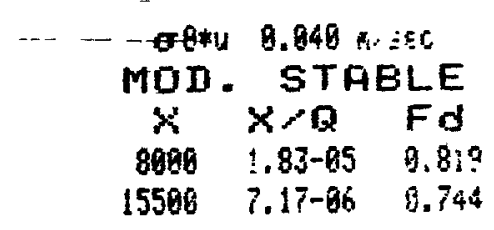


MATHEMATICAL MODELS USED WITHIN "X/Q"

A. Hanford very stable and moderately stable.

$$
\sigma_{y}^{2}=A\left[t+\alpha\left(e^{-t / \alpha}-1\right)\right]
$$

where

$$
\begin{aligned}
& t=x / u, x \text { being the downwind distance and } u, \text { the wind speed } \\
& A=13+232\left(\sigma_{\theta} u\right) \\
& \alpha=\frac{A}{2\left(\sigma_{\theta} u\right)^{2}}
\end{aligned}
$$

If

$$
\mathrm{u}=1 \mathrm{~m} / \mathrm{s},
$$

then

$\sigma_{\theta} u$ is computed from the release duration as follows:

$$
\sigma_{\theta} u=(\text { release duration }) / 3000+.02
$$

such that

$$
\begin{aligned}
& .024 \leq \sigma_{\theta} u \leq .18 \\
& \sigma_{z}^{2}=a\left(1-e^{-k^{2} t^{2}}\right)+b t
\end{aligned}
$$


Values for $a, b$, and $k^{2}$ are listed below:

\begin{tabular}{|c|c|c|}
\hline Parameter & Very stable & Moderately stable \\
\hline$a$ & $34 \mathrm{~m}^{2}$ & $97 \mathrm{~m}^{2}$ \\
\hline b & $.025 \mathrm{~m}^{2} / \mathrm{s}$ & $.33 \mathrm{~m}^{2} / \mathrm{s}$ \\
\hline$k^{2}$ & $.00088 \mathrm{~s}^{-2}$ & $.00025 \mathrm{~s}^{-2}$ \\
\hline
\end{tabular}

B. Sutton's neutral and unstable.

$$
\sigma_{y}^{2}=\frac{1}{2} C_{y}^{2} x^{(2-n)} \text { and } \sigma_{z}^{2}=\frac{1}{2} C_{z}^{2} x^{(2-n)} \text { and } \sigma_{z}<2000 m
$$

C. Building wake effects.

$$
\begin{gathered}
\sigma_{y} \text { and } \sigma_{z} \text { are replaced with } \Sigma_{y} \text { and } \Sigma_{z} \text { as follows: } \\
\Sigma_{y}^{2}=\sigma_{y}^{2}+\frac{S}{2 \pi} \text { and } \Sigma_{y}^{2}=\sigma_{z}^{2}+\frac{S}{2 \pi}
\end{gathered}
$$

such that

$$
\Sigma_{y} \Sigma_{z} \leq 3 \sigma_{y} \sigma_{z}
$$


D. Integrated ground deposition loss.

$F_{d}$ is the fraction remaining at distance $x$.

$1-F_{d}=$ fraction lost to ground deposition.

$$
\ln F_{d}=-\sqrt{\frac{2}{\pi}}^{\frac{d}{u}} \int{ }_{0}^{x} \frac{d x}{\Sigma_{z}} \exp \left(\frac{-h^{2}}{2 \Sigma_{z}^{2}}\right)
$$

Typical values for $v_{d}$ are as follows:

$0.1 \mathrm{~cm} / \mathrm{s}$ for most particulate matter

$$
1.0 \mathrm{~cm} / \mathrm{s} \text { for halogens (e.g., iodine). }
$$

E. Time-integrated air concentration.

1. Centerline $X / Q$

$$
\frac{X}{Q}=\frac{F_{d}}{\pi u \Sigma_{y} \Sigma_{z}} \exp -\left(\frac{-h^{2}}{2 \Sigma_{z}^{2}}\right)
$$

2. Sector-averaged $X / Q, 22.5$ degree sectors

$$
\frac{X}{Q}=\frac{8 F \mathrm{~d}}{\pi u \times \Sigma z} \quad \sqrt{\frac{2}{\pi}} \exp \left(\frac{-h^{2}}{2 \Sigma_{z}^{2}}\right)
$$

Sector-averaged $X / Q$ values are normally used when determining population dose or when the release duration exceeds $8 \mathrm{~h}$. 
MEMORY USE:

00: distance pointer

01: integration pointer

02: u (wind speed)

03: integration $x$

04: $x$ increment

05: $\Sigma_{z}^{2}$

06: $\Sigma_{y}^{2}$

07: $2-n$

08: $C_{y}$

09: $C_{Z}$

10: $\left(\Sigma_{y} / \sigma_{y}\right)^{2}$

11: $\left(\Sigma_{z} / \sigma_{z}\right)^{2}$

12: $S / 2 \pi$

13: piecewise sum

14: overall integration sum

15. $C_{y} N$

16. $\mathrm{C}_{Z} \mathrm{~N}$

17: $v_{d}$ (depletion speed)

18: A

19: $\alpha$

20: $h$ (release height)

21: $C_{y}, C_{z}$ UN or specific MET

22: distance pointer save

23: 1st distance

24: 1st population

25: 1st $(X / Q)$ (population)
USER MODE LABELS:

$$
\begin{aligned}
& \text { A: input new } h \\
& \text { B: input new } u \\
& C \text { : input new } x \text { values (and } \\
& \text { populations, if sector } \\
& \text { averaging) } \\
& \text { D: input new MET choice and } \\
& \text { run } \\
& \text { E: execute program } \\
& F: \quad \text { input new } v_{d} \\
& \text { G: } \quad \text { input new } S \\
& \mathrm{H} \text { : choose or remove sector } \\
& \text { averaging (sets flag } 03 \\
& \text { if clear and clears }
\end{aligned}
$$

FLAG USE :

$00: \quad v_{d}=0.0 \mathrm{~cm} / \mathrm{s}$

01: $u=1.0 \mathrm{~m} / \mathrm{s}$

02: $\quad S>0 \mathrm{~m}^{2}$

03: sector averaging

04: MET is one of Sutton's forms ( $N$ or UN)

05 MET is UN or MS

06: all 4 stability classes are run

07:) These 3 flags sequence 08: the stability classes

09: when all four classes are run

10: used in the building wake logic 


\section{PROGRAM LISTING}

$$
\begin{aligned}
& \text { LEL } \\
& \text { EFI } 1 \text { DEE ENTES }
\end{aligned}
$$

\begin{tabular}{|c|c|}
\hline \multicolumn{2}{|l|}{ 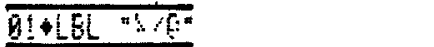 } \\
\hline $\bar{B} 2 \mathrm{CF}=\bar{i}$ & \\
\hline $63 \mathrm{CF}^{4}$ & \\
\hline $94+28-46$ & SPEEDS USER MODE \\
\hline 856929 & TO END AT THE \\
\hline at $\left.5 T 0^{\circ}\right)$ & \\
\hline$\theta^{n}{ }^{\prime} H=? n^{n}$ & \\
\hline
\end{tabular}

6E PI 20 -

99 PK, MPT

$\overline{I B+L B L} \bar{H}$

1! STก 20

12 FS? 27

13 GT0 45

14 XEE 64

15 "I $=7$ M.S"

$16 \mathrm{RCL}$ B2 - AECALLS PmeVOUs

17 PROHFT

$\overline{18+L B L E}$

14 STी

20 CF Bi

211

$22 \mathrm{y}=\mathrm{Yr}$

23 $\mathrm{SF} Q \mathrm{i}$

24 F5? 27

25 gTO 46

26 YEO 65

$2 \vec{i}=\mathrm{Hd}=2 \mathrm{CH} . \mathrm{i}$

$26 \mathrm{OAX}-$ ZERO WLL BE
29 PFIMPT

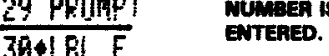

$31 \mathrm{CF} \overline{\mathrm{B}}$

$32 y=y^{2}$

$33 \mathrm{SF} \mathrm{BA}$

34 STI 17

$35 \mathrm{FS} 27$

36 GTi 45

37 XEF

38 "BLDC. $99 \mathrm{gF}$ '

$39 \mathrm{Ax}$

46 PFInPT

$41+\angle E_{-15}$

42 6 $1 \%$

432

$44:$

$45 \div$

$45:$

$4 \mathrm{SiC}^{2}: 5$

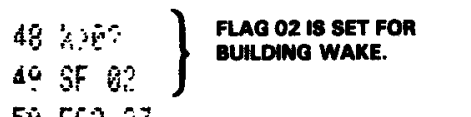

$54 \mathrm{FS}^{2} \mathrm{Z}$

51 GTE 46

52 XE? $B ?$

53 CF $2:$

$54 \mathrm{CF} 63$

55 -SECTOP AVE. $\because$

56 PROHFT

$57 \mathrm{Fin}^{22}$

$58670 \mathrm{C}$

$59+1 B L F$

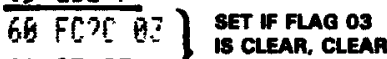

6) SF 93 , If CLET.

$62 F 9 ? 27$

63 GTI 46

$64+L B L C=$ DISTANCE AND

651.1

66 STO 98

67 STO 33

6222

$59+$

79 ST0 04

$71+L B L 06$

72 FIR

73 ष

74 ARCL QG

$75 \circ=7 H^{\circ}$

76 CLX

77 PROMPT

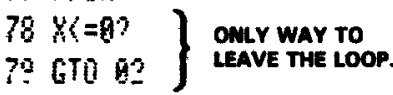

30 STO IHE 64

913

62 FI? 937 skIPs population

Q3 GTII $9:\}$ PROM BECTOH

84 Fi:

85 IfG 64

86 "fop."

8. API: BP

88 it 7

291

90 PRINP:

$915 T 0$ IND 4

92

$93+18: b_{4}^{2}$

$945 T+24$

95150

$960 \mathrm{~T}$ G

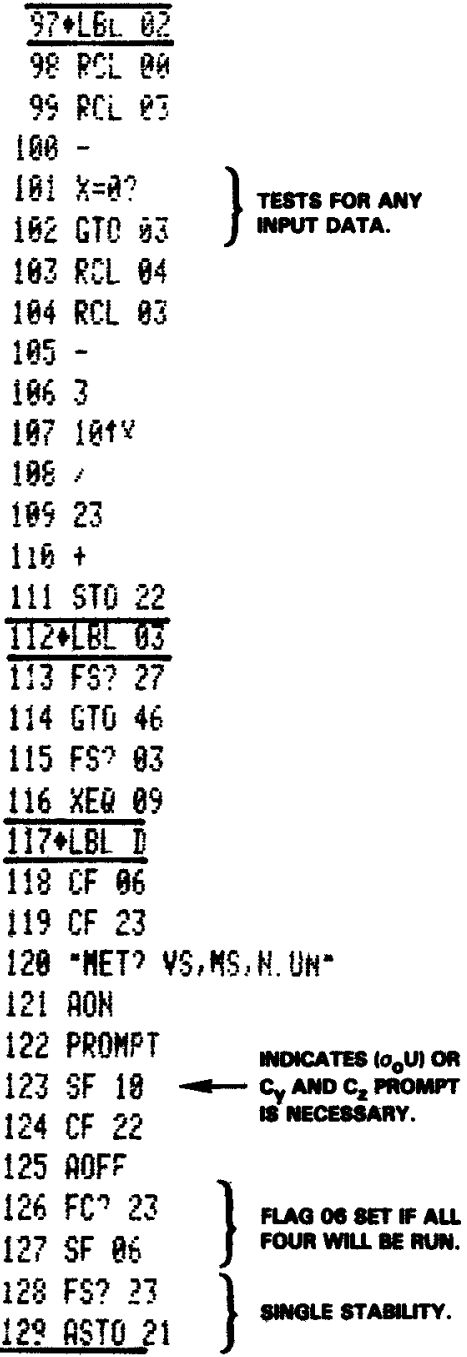

$139+\angle B L E$

131 OF 85

132 CF 67

137 CF 09

134 CF 80

135 FC? 66

136 GTO IHD 24

137 FC? 10

i39 6T0 0!

139 "CY H ?"

149 PROMFT

141 FC? $2 E$

$1426 T 0$ :

14351015

$144^{\circ} \mathrm{CZ} \mathrm{H}$ \%"

145 PROMP?

$1465 T \div 16$ 


\section{PROGRAM LISTING}

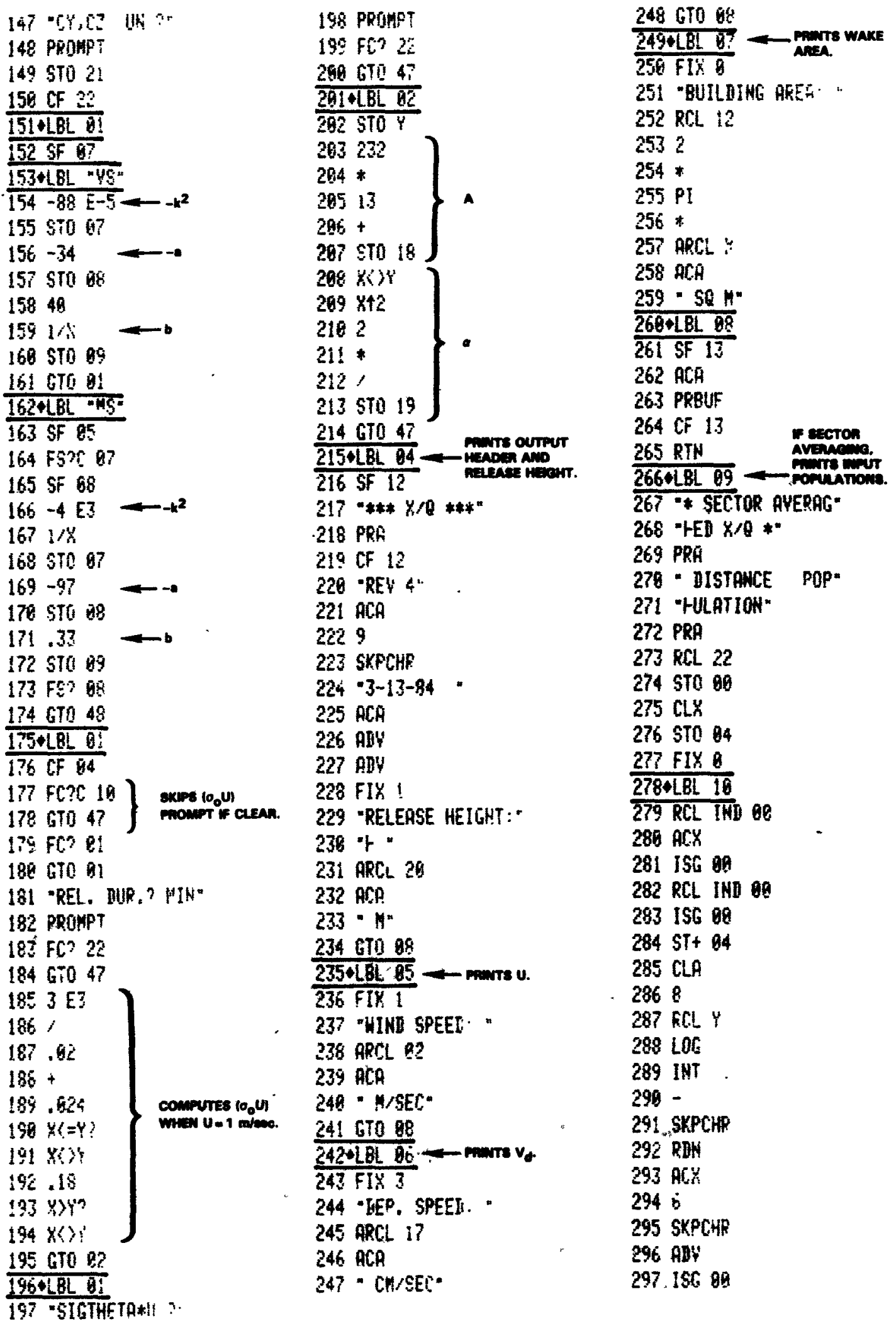




\section{PROGRAM LISTING}

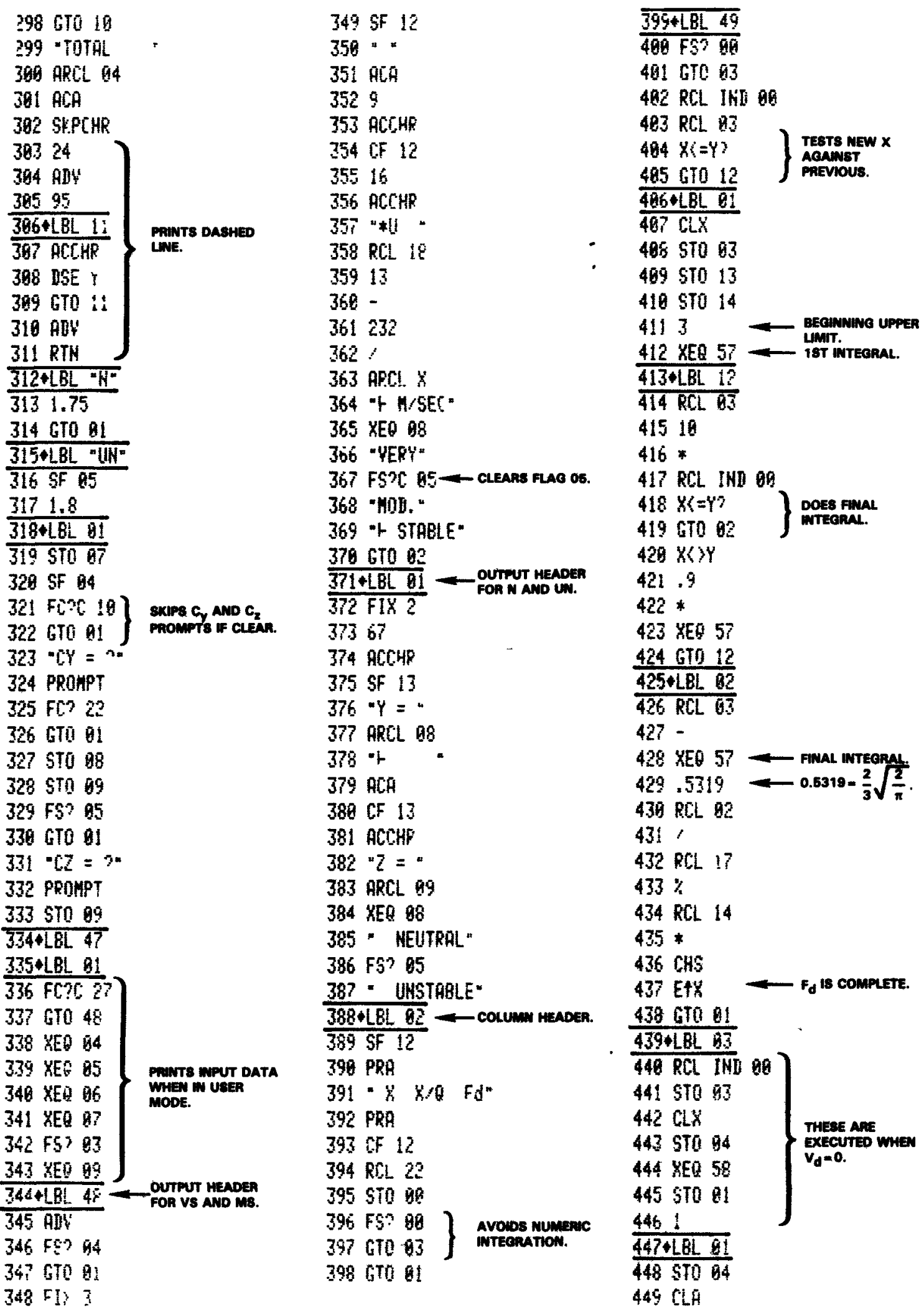




\section{PROGRAM LISTING}

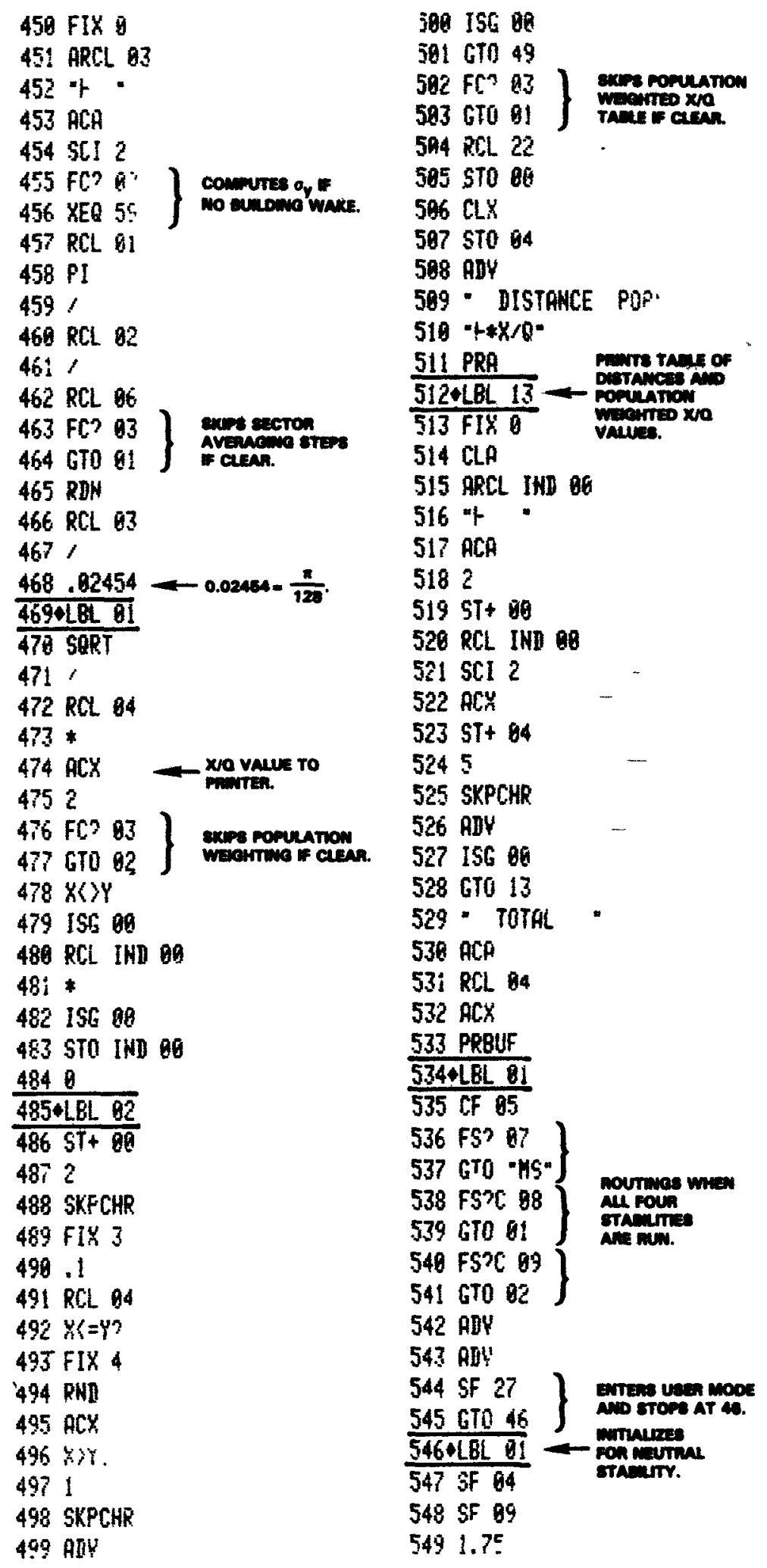

550 STO 07

$55 ! \mathrm{RCL} 15$

$5525 T 0 \quad 68$

553 RCL 16

554 STO 99

$555 \quad 67048$

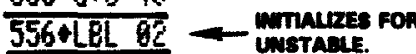

557 SF 65

5581.8

559 STO 67

$56 \mathrm{ACL} 21$

561 sT0 98

562 sTo 69

563 GTO 48

$564+\mathrm{LBL} 57$

565 10

566

$5675 T 084$

5681.01

569 STO 01

578. LBL 14

571 XEQ 58

$572 \mathrm{ST}+13$

573 IS6 01

$574 \times 5058^{-}$

575 ISG 01

576 6T0 14

577 STO 01

5782

579 !

589 ST- 13

$581 X<>13$

$582 \mathrm{RCL}$

$563 *$

$584 \mathrm{ST}+14$

585 RTN

$586+$ LBL 59

$587 \mathrm{RCL} 12$

588 RCL 63

$589 \mathrm{FS}^{2}$.

59865061

$591 \mathrm{RCL} \quad 82$

59 :

593 EHTER*

594 ENTER

$595 \mathrm{RCL} 19$

$596 /$

$597 \mathrm{CHS}$

$598 \mathrm{EHX}-\mathrm{t}$

599 RCL 19

$699 *$

6月: +

692 RCL 18

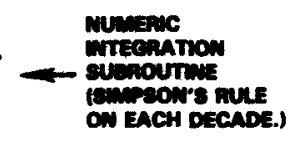

Immoowte mure

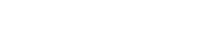




\section{RHO-HS-ST-5 P .}

\section{PROGRAM LISTING}

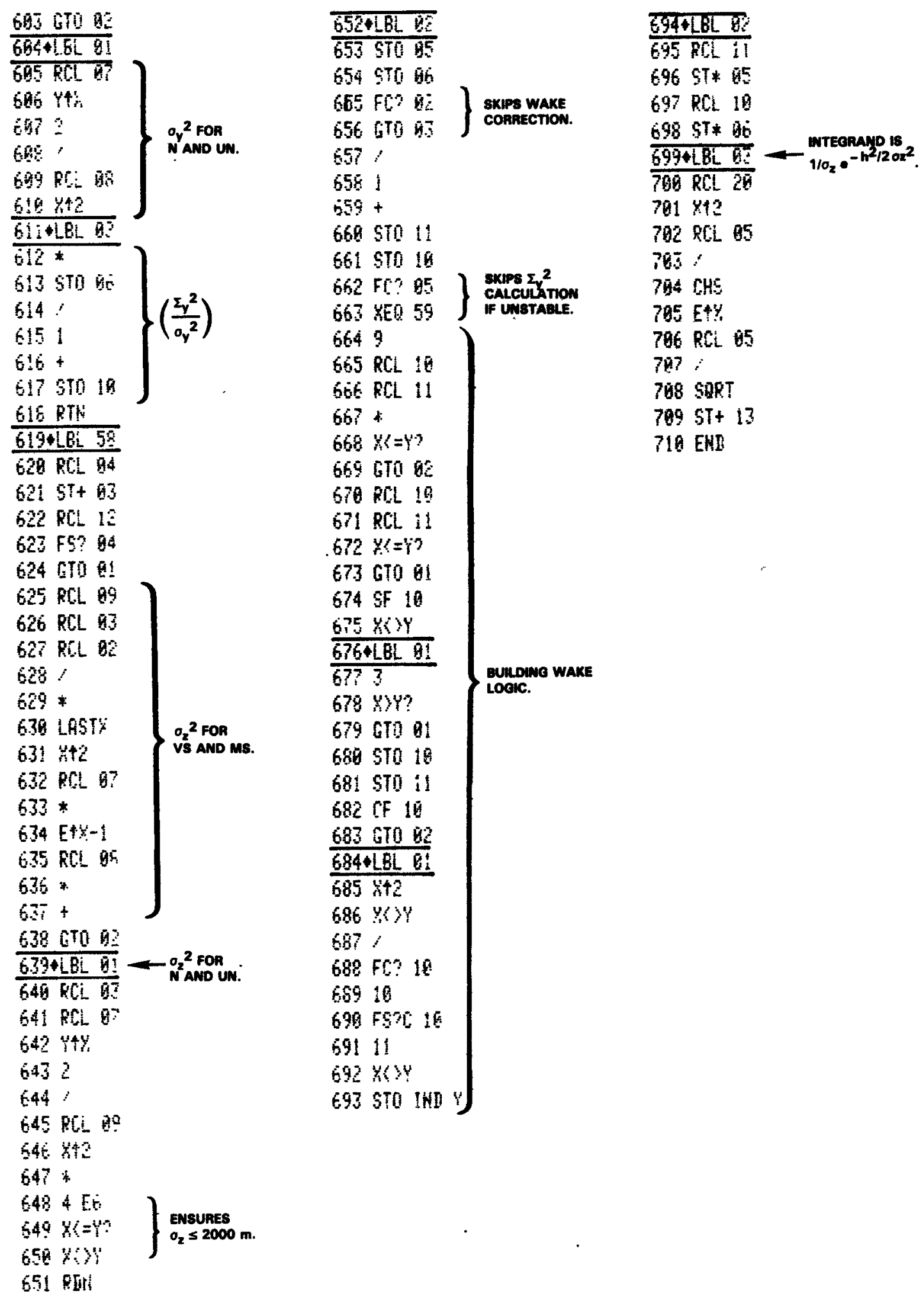


RHO-HS-ST-5 P

APPENDIX F

EMERGENCY RESPONSE - "P/Q" 



\section{USER INSTRUCTIONS}

A. Enter program (read three cards).

B. Press XEQ "P/Q." Program may be run with or without a printer. If the printer is off or not attached, all displayed results will stop program execution. To continue, simply press R/S.

C. Input requested information (1isted here), pressing $R / S$ after each entry.

1. Release height $(h)$ in meters.

2. Wind speed at the point of release (u) in meters/second.

3. Downwind distance to receptor $(x)$ in kilometers.

4. If sector averaging (22.5 degree sectors) is desired, enter any number. For center 1 ine $X / Q$, do not enter any number.

5. Stability class - enter one of the PG classes, A through F.

D. Once $X / Q$ has been computed, the program asks "INPUT LIST?," which means the option to review input parameters and see the computed values of $\sigma_{y}$ and $\sigma_{z}$ is available. If a listing is desired, enter any number and press $R / S$. Press $R / S$ to view successive parameters if no printer is attached or if the printer is off. If the listing is not desired, enter nothing; just press R/S.

NOTE: When sector averaging, the parameter $\sigma_{y}$ is replaced with the quantity $(125 x) \sqrt{\pi} / 2$.

E. The next option facilitates dose computation for each isotope using unit dose factors and curies released.

- If such $a_{3}$ computation will be done, enter the first dose factor $(\mathrm{rem} / \mathrm{Ci} \cdot \mathrm{m} / \mathrm{s})$ and the activity released for each isotope. The program computes the dose from each isotope and displays it. Once a11 isotopes have been computed, the program prompts for another dose factor. Press $R / S$ and the sum tota 7 will be displayed.

- If no dose computation is desired, enter nothing; just press R/S and "P/Q" will execute from the beginning. 
F. Finally, the user may simply want to change one or two input parameters and rerun the $X / Q$ calculation. This capability is realized in two ways:

1. Switch to USER mode.

a. To change $h$, enter the new $h$ and press $A$.

b. To change $u$, enter the new $u$ and press $B$.

c. To change $x$, enter the new $x$ and press $C$.

d. To switch to sector averaging or to remove this option, press $\mathrm{H}$.

e. To change stability class, press D. The MET choices will then be displayed. Enter the new MET and press R/S. The program will store and execute the new MET.

f. To execute the program, press $E$.

2. Execute "P/Q" from the beginning.

a. As each item is prompted, enter either the new value and press $R / S$ or the previous value by pressing only R/S.

b. Previous values of $h, u$, and $x$ are recalled to the $x$-register just prior to their prompt and may be viewed by clearing the display. Centerline $X / Q$ will be computed unless sector averaging is requested.

G. Mathematical formulas are listed in the "X/Q - Pasquill" program instructions. 
MEMORY USE :

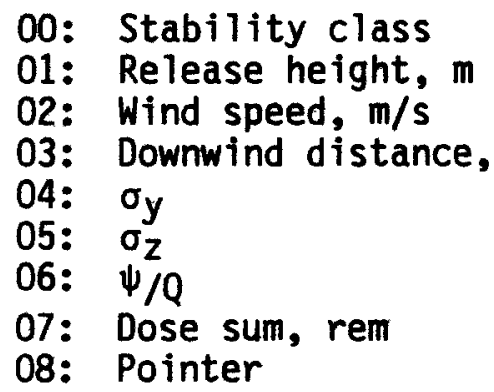

\section{PROGRAM LISTING}

LEL $\times P>Q 9$
EHI

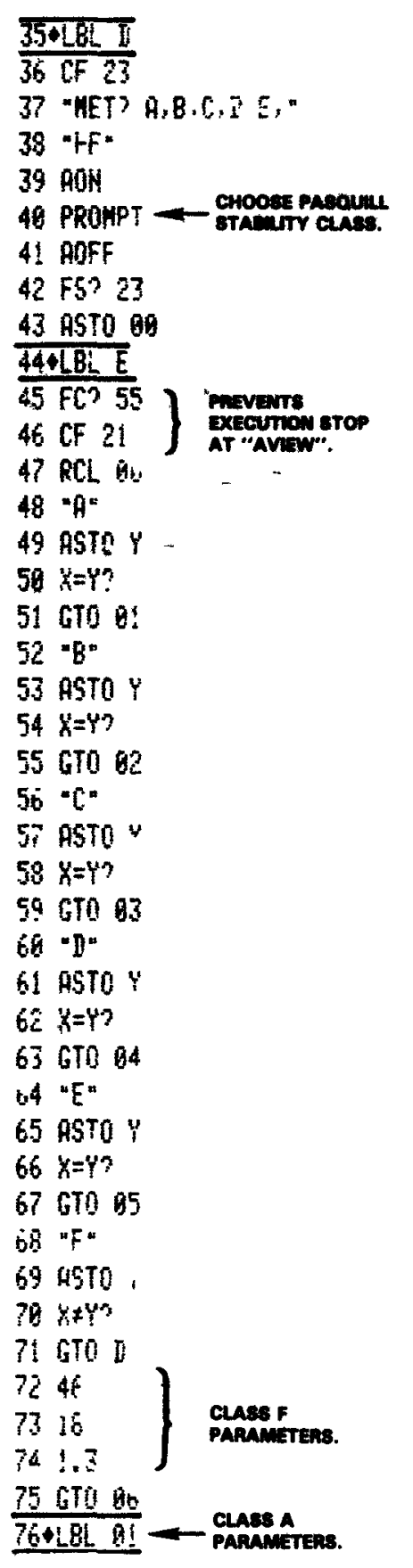

FLAG USE:

00: Sector averaging

77240

78146

792.7

$80 \mathrm{RCL} 03$

81 GTO 60

824LBL BE - CLASS B

83185

24162

85.26

$36 \mathrm{RCL} 93$

87 SORT

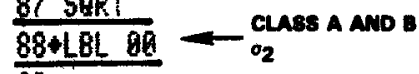

g!*

$90 \mathrm{RCL} 05$

$91 *$

921

$93+$

$94 *$

$35 \mathrm{RCL}-3$

96:

9767004

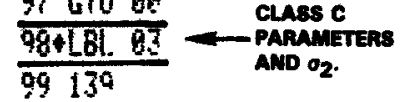

$100 \mathrm{RCL} \quad 03$

161.3

$102 \mathrm{RCL} B Z$

103 SQPT

$104 *$

1651

$186+$

$107 \%$

16883

$109 *$

110 GT0 B⿺

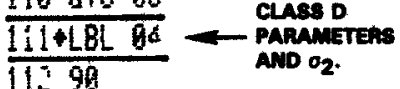

$113 \mathrm{RCL} \mathrm{B}$ ?

1141.1

$115 R_{+} 6 ?$

:16*

II?:

$119+$

$1: 9$ SOFT 


\section{PROGRAM LISTING}

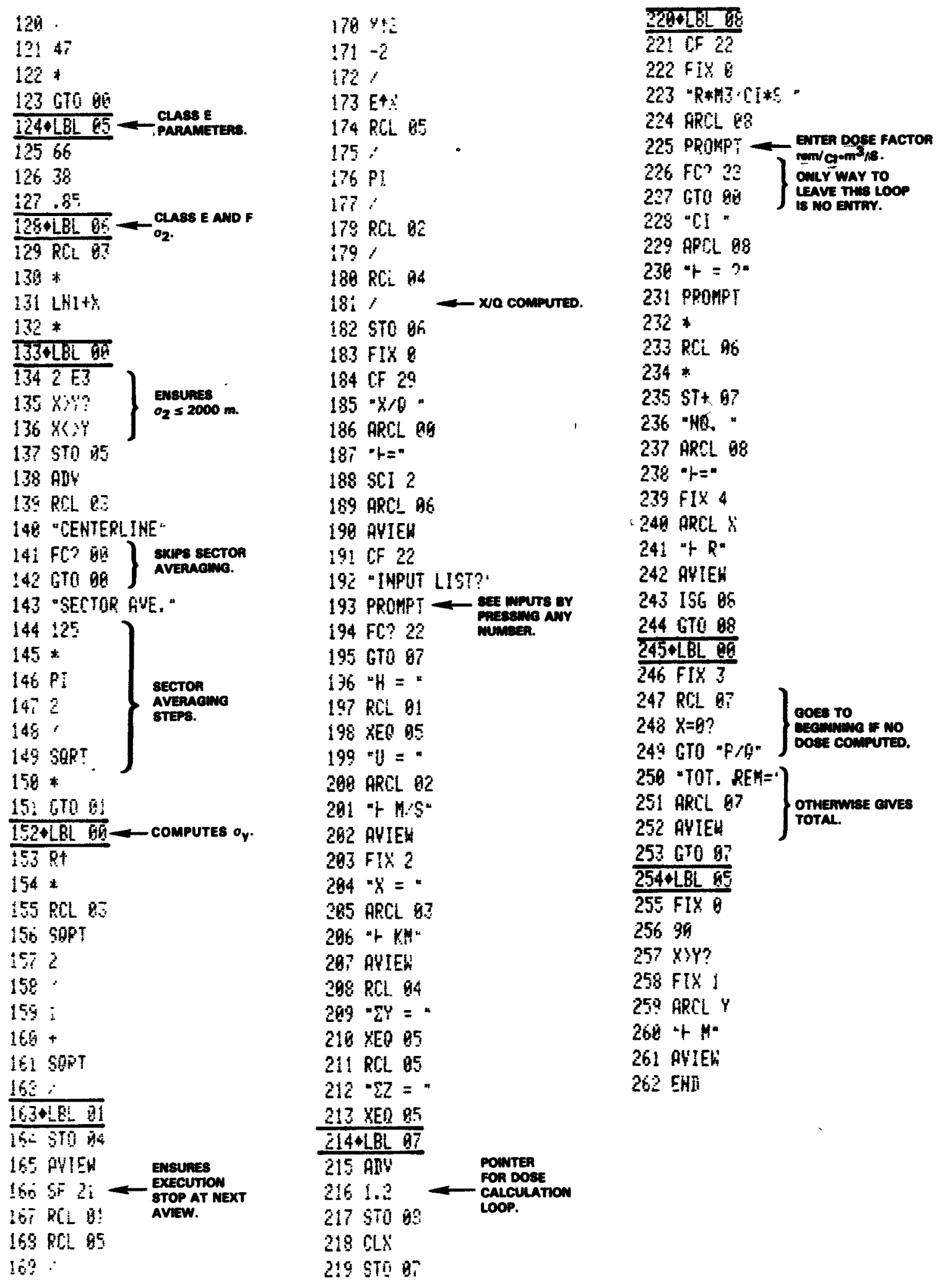


RHO-HS-ST-5 P

APPENDIX G

"X/Q - PASQUILL" 



\section{USER INSTRUCTIONS}

A. Attach printer and partition calculator memory as follows before reading in the program (6 cards): SIZE $=20+3 \mathrm{~N}$, where $\mathrm{N}$ is the number of downwind distances to be used. In the standard HP-41CV, the maximum SIZE allowed with "X/Q" is 122 , which allows 37 distances.

B. Execute " $X / Q$ " and enter the necessary information (listed here), pressing $R / S$ after each input.

1. Release height $(h)$ in meters.

2. Wind speed $(u)$ in meters per second.

3. Deposition speed $\left(v_{d}\right)$ in centimeters per second. Zero is automatically entered if only $R / S$ is pressed.

4. Building areas $(S)$ in square meters. If building wake effects will not be included, simply press $R / S$ without entering a number. Zero area is automatically entered.

5. If sector averaging is desired, enter any number. To leave out the sector-average option, enter nothing; just press $R / S$.

6. The first distance to be used. The program then prompts for each distance. If sector averaging is selected, the program also prompts for the population at each distance. Please bear in mind the following:

a. To reduce program running time, enter distances in increasing order.

b. Zero is not an allowed distance.

c. Unit populations are automatically entered if no other values are input by the user.

d. Distance (and population) data are not altered by program execution. If data from the previous run (or data card entry) will be used, press $R / S$ when the first distance prompt appears.

When all distances have been entered, press $R / S$ at the next distance prompt.

7. Pasquill-Gifford atmospheric stability class. Because the calculator is not in ALPHA mode, simply enter the appropriate letters or letters up to a maximum of six. Each stability will be computed in the order entered. 
C. Once the output is complete, the input data may be changed and the program run again. To restart from the beginning, press R/S. To change only a few items and execute, switch to USER mode and input the changes using local alpha labels as follows:

1. To change $h$, enter the new $h$ and press $A$.

2. To change $u$, enter the new $u$ and press $B$.

3. To change $v_{d}$, enter the new $v_{d}$ and press $F$.

4. To change $S$, enter the new $S$ and press $G$.

5. To switch to sector-averaged $X / Q$ or to remove this option, press $H$. Be sure to enter population data, if necessary.

6. To change distances (and populations if sector averaging), press C and enter the requested information.

7. To change stability class or classes, press $D$. The program begins executing with the "MET? A, B, C, D, E, F" prompt. Enter your selections according to the instructions given in step B.7.

8. To execute a run without changing stabilities, press $E$. 


\section{RHO-HS-ST-5P}

\section{SAMPLE OUTPUT}

The printout on the left below was obtained using sector averaging and selecting PG classes $D$ and $E$.

The printout shown on the right was generated by clearing the sectoraverage option and choosing all six classes.

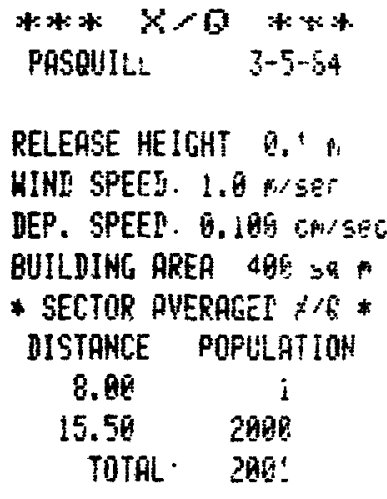

FASDUILL I

$x$ Po Fd

$8.80 \quad 1.81-06 \quad 9.65 t$

15.58 6.23 -8.82.

$$
\begin{array}{cc}
\text { DISTANCE } & \text { PDP } \times 19 \\
8.06 & 1.81-3) \\
15.59 & 1.25-3 ? \\
\text { TOTAL } & 1.26-96
\end{array}
$$

FASTUILL E $\therefore \quad X 0$ Fo

$8.09 \quad 2.61-96 \quad 0.898$

15.59 $\quad 9.89-9^{7} \quad 9.756$

$$
\begin{array}{cc}
\text { DISTANCE POP* T } \\
8.99 & 2.61-65 \\
25.50 & 1.96-65 \\
\text { TOTAL } & 1.96-63
\end{array}
$$

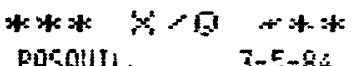

$$
\begin{aligned}
& \text { PA50UILL 7-F-84 } \\
& \text { RELEASE HEIGH }{ }^{\top} \text {. } 1 * \\
& \text { HIND SPEEI } 1.0 \mathrm{~A} \text {. } \\
& \text { DEP. SPEEI 0.160 CAYSEC } \\
& \text { BUILDING DREA } 4 \text { GG SA A } \\
& \text { PASBUILL F } \\
& X \quad X<D \text { Fd } \\
& 8.69 \text { 2.29-8E 5.? } \\
& 59.50 \quad 8.99-9 \mathrm{n} \quad 0.544 \\
& \text { FASAUILLE } \\
& X \text { X } P \text { Fa } \\
& \text { 6.80 } 9.64-150 \quad 0.803 \\
& 15.58 \text { 4.01-0. } 0.756 \\
& \text { PASQUII: II } \\
& \therefore \text { XO FU } \\
& 6.60 \text { 4.85- Br } 5.50 \\
& 15.59 \quad: .8 \bar{x}-6.0 .8 \div
\end{aligned}
$$

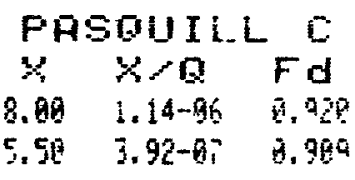

$$
\begin{aligned}
& \text { FASQUILL E } \\
& \because x<0 \text { Fo } \\
& 8.60 \text { 1.59-0. } 0.957 \\
& 15.56 \quad 9.89-98 \quad 6.456 \\
& X \text { X } \mathrm{F} \text { F } \\
& 8.90 \text { 1.25- } \\
& 15.50 \quad 7.15-960.98
\end{aligned}
$$


MATHEMATICAL MODELS USED BY "X/Q - PASQUILL"

A. Pasquill-Gifford curves for $\sigma_{y}$ and $\sigma_{z}$ are well approximated by the formulas given below, where $x$ is in $\mathrm{Km}$.

TABLE 7. Pasquil1-Gifford Curves.

\begin{tabular}{c|c|c}
\hline $\begin{array}{c}\text { Stability } \\
\text { class }\end{array}$ & $\sigma_{y}=\mathrm{a} \times /(1+.5 \sqrt{\mathrm{x}})^{\frac{1}{2}}$ & $\sigma_{z^{\star}}$ \\
\hline A & $\mathrm{a}=240$ & $146 \times\left(1+2.7 \times x^{2}\right)$ \\
$\mathrm{B}$ & $\mathrm{a}=185$ & $102 \times(1+.26 \times \sqrt{\mathrm{x}})$ \\
$\mathrm{C}$ & $\mathrm{a}=139$ & $83 \times /(1+.3 \sqrt{\mathrm{x}})$ \\
$\mathrm{D}$ & $\mathrm{a}=90$ & $47 \times /(1+1.1 \mathrm{x})^{\frac{1}{2}}$ \\
$\mathrm{E}$ & $\mathrm{a}=66$ & $38 \ln (1+.85 \mathrm{x})$ \\
$\mathrm{F}$ & $\mathrm{a}=46$ & $18 \ln (1+1.3 \mathrm{x})$ \\
\hline
\end{tabular}

NOTE: Plots of these functions are shown in Figure 4.

*The vertical dispersion parameter is not allowed to exceed 2,000 $\mathrm{m}$.

B. Building wake effects.

$$
\begin{aligned}
& \sigma_{y} \text { and } \sigma_{z} \text { are replaced with } \Sigma_{y} \text { and } \Sigma_{z} \text { as follows: } \\
& \Sigma_{y}^{2}=\sigma_{y}^{2}+\frac{S}{2 \pi} \quad \text { and } \quad \Sigma_{z}^{2}=\sigma_{z}^{2}+\frac{S}{2 \pi}
\end{aligned}
$$

such that $\Sigma_{y} \Sigma_{z} \leq 3 \sigma_{y} \sigma_{z}$ 

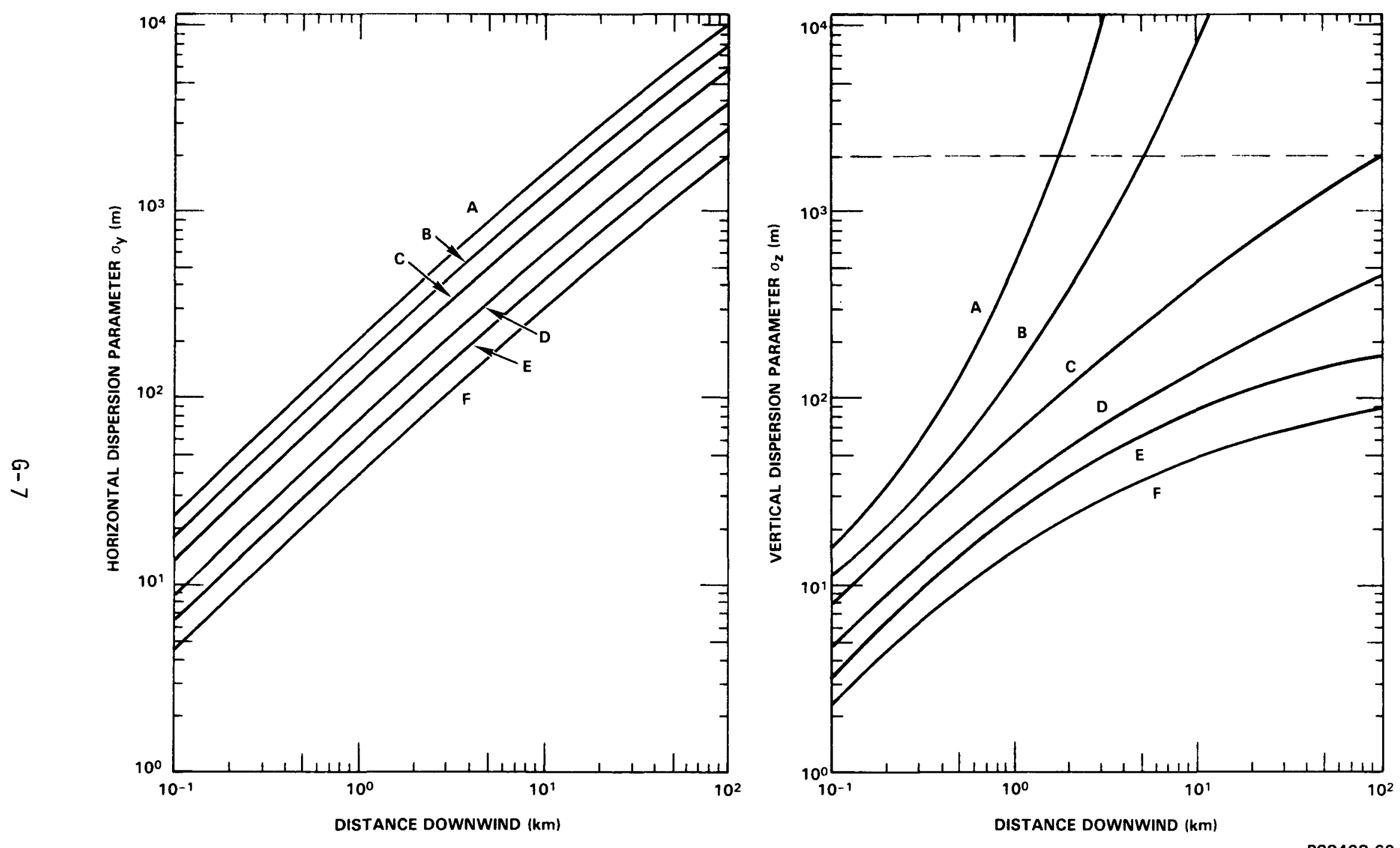

FIGURE 4. Plots of the Pasquil1-Gifford Curves as Parameterized for "X/Q - Pasquill." 
C. Integrated ground deposition loss.

$F_{d}$ is the fraction remaining at distance $x$.

$1-F_{d}=$ fraction lost to ground deposition.

$$
\text { ln } F_{d}=\sqrt{\frac{2}{\pi}} \frac{v_{d}}{u}\left(\int_{0}^{x} \frac{d x}{\Sigma_{z}} \exp \frac{-h^{2}}{2 \Sigma_{z}^{2}}\right) \frac{10 m^{2}}{c m-K m}
$$

Typical values for $v_{d}$ are as follows:

$0.1 \mathrm{~cm} / \mathrm{s}$ for most particulate matter

$1.0 \mathrm{~cm} / \mathrm{s}$ for halogens (e.g. iodine).

D. Time integrated air concentration.

1. Center 1 ine $X / Q$

$$
\frac{X}{Q}=\frac{F d}{\pi u \Sigma y_{z}} \quad \exp \left(\frac{-h^{2}}{2 \Sigma_{z}^{2}}\right)
$$

2. Sector-averaged $X / Q, 22.5$ degree sectors

$$
\frac{\mathrm{X}}{\mathrm{Q}}=\frac{.008 \mathrm{~F}_{\mathrm{d}}}{\pi \mathrm{ux \Sigma} z} \quad \sqrt{\frac{2}{\pi}} \quad \exp \left(\frac{-\mathrm{h}^{2}}{2 \Sigma_{z}^{2}}\right)
$$

Sector-averaged $X / Q$ values are normally used when dose population is determined or when the release duration exceeds $8 \mathrm{~h}$. 
MEMORY USE :

00: distance pointer

01: integration pointer

02: u (wind speed)

03: integration $x$

04: $x$ increment

05: $\Sigma_{z}^{2}$

06: $\quad \Sigma_{y}^{2}$

07: A

08: b

$\sigma_{Z}$

09: a

10: $\left(\Sigma_{y} / \sigma_{y}\right)^{2}$

11: $\left(\Sigma_{z} / \sigma_{z}\right)^{2}$

12: $S / 2 \pi$

13: piecewise sum

14: overall integration sum

15: MET save

16: MET remaining

17: $v_{d}$ (depletion speed)

18: h (release height)

19: distance pointer save

20: 1st distance

21: 1st population

22: 1 st $(X / Q)$ (population)
USER MODE LABELS:

A: input new $H$

B: input new $u$

C: input new $x$ values (and populations if sector averaging)

$D$ : input new MET choice and run

E: execute program

$F$ : input new $v_{d}$

G: input new $S$

$\mathrm{H}$ : choose or remove sector averaging (sets flag 02 if clear, and clears flag 02, if set)

FLAG USE:

00: $\quad v_{d}=0.0 \mathrm{~cm} / \mathrm{s}$

01: $\mathrm{s}>0 \mathrm{~m}^{2}$

02: sector averaging

TABLE 8.

Flags Used to Define the MET Class.

Flags

\begin{tabular}{c|c|c|c}
\hline MET & 03 & 04 & 05 \\
\hline A & & & \\
B & & & Set \\
C & & Set & \\
D & & Set & Set \\
E & Set & & \\
F & Set & Set & \\
\hline
\end{tabular}


PROGRAM LISTING

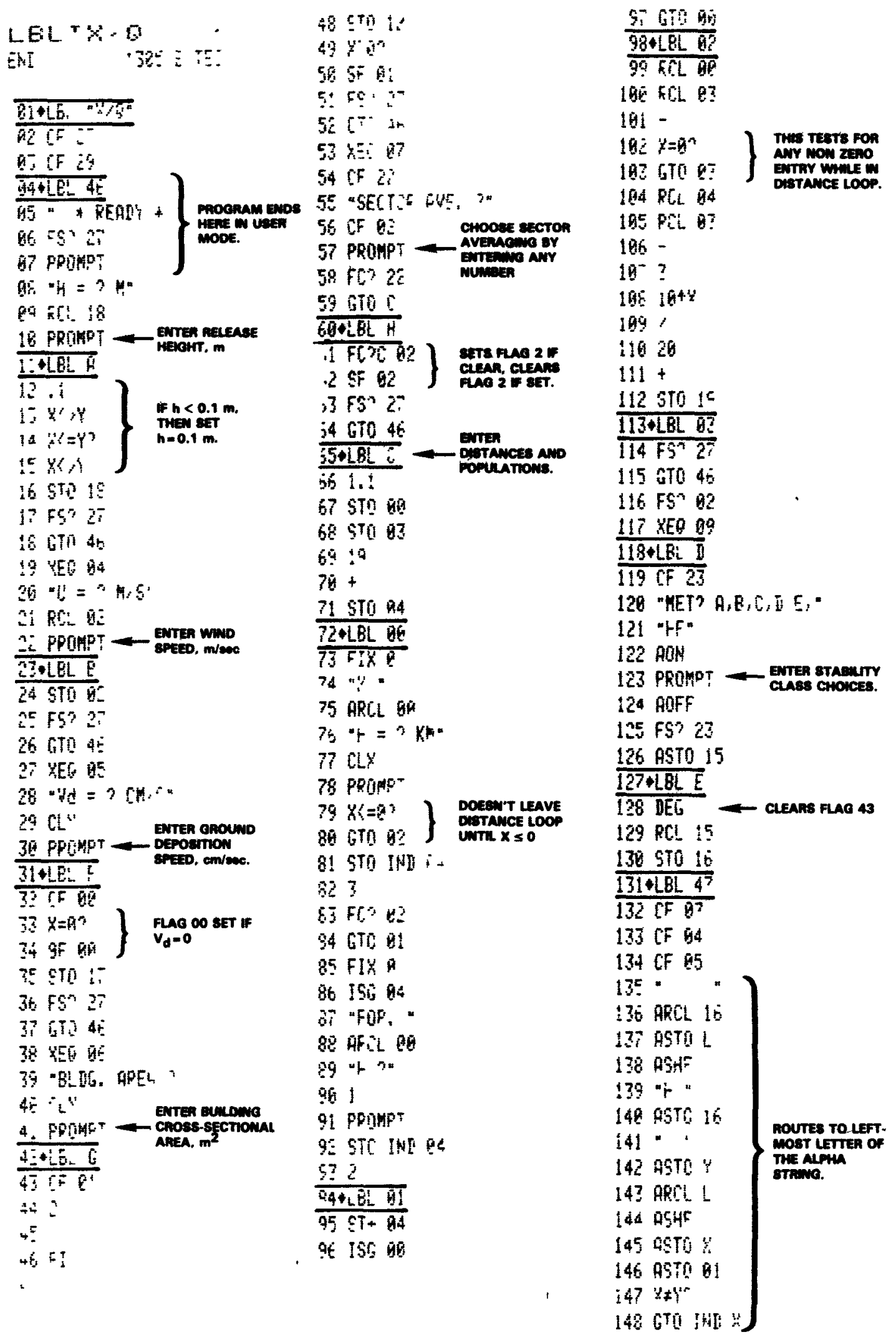




\section{PROGRAM LISTING}

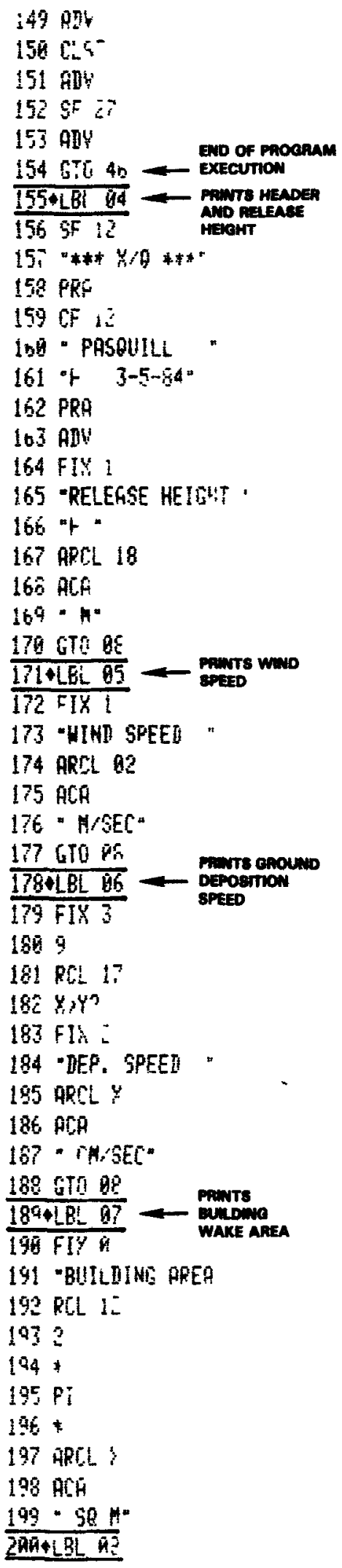

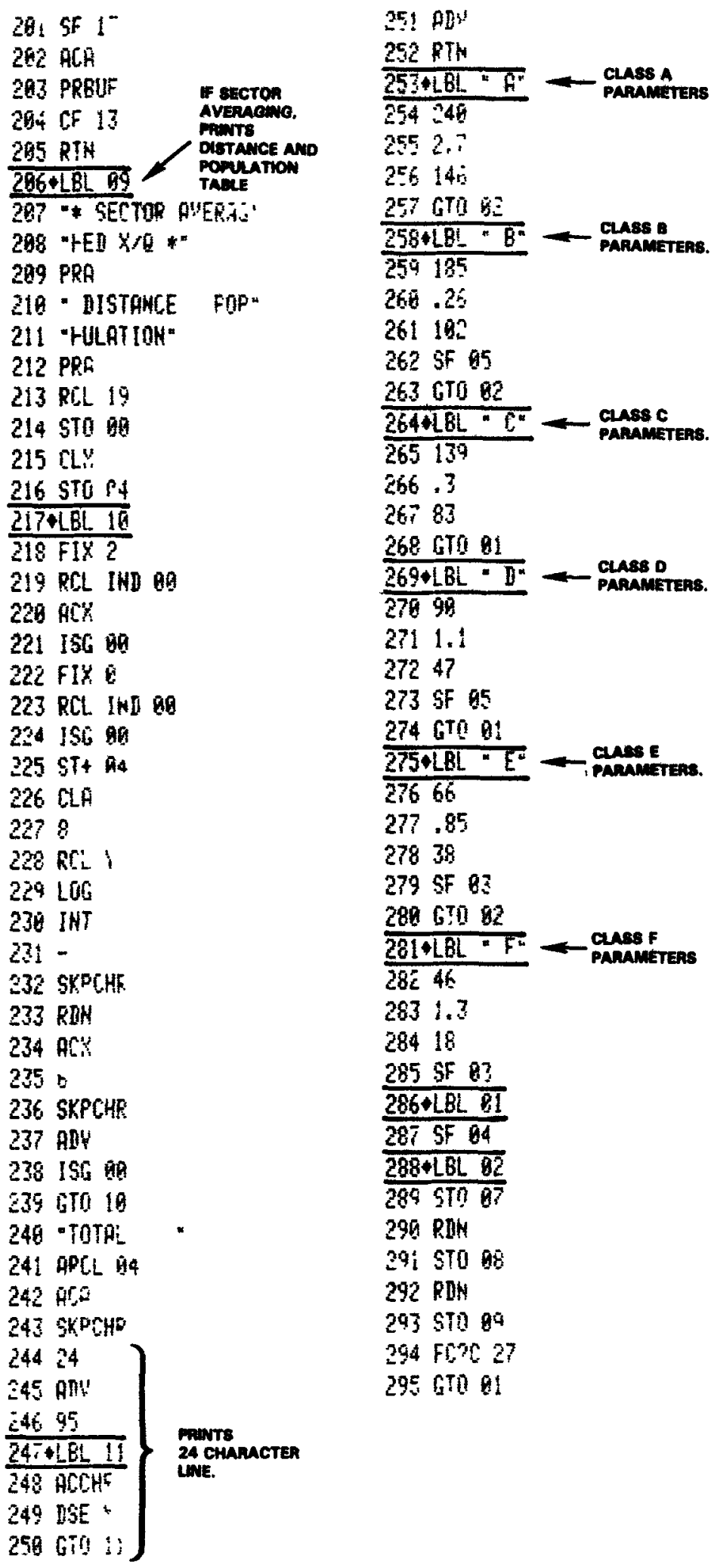




\section{PROGRAM LISTING}

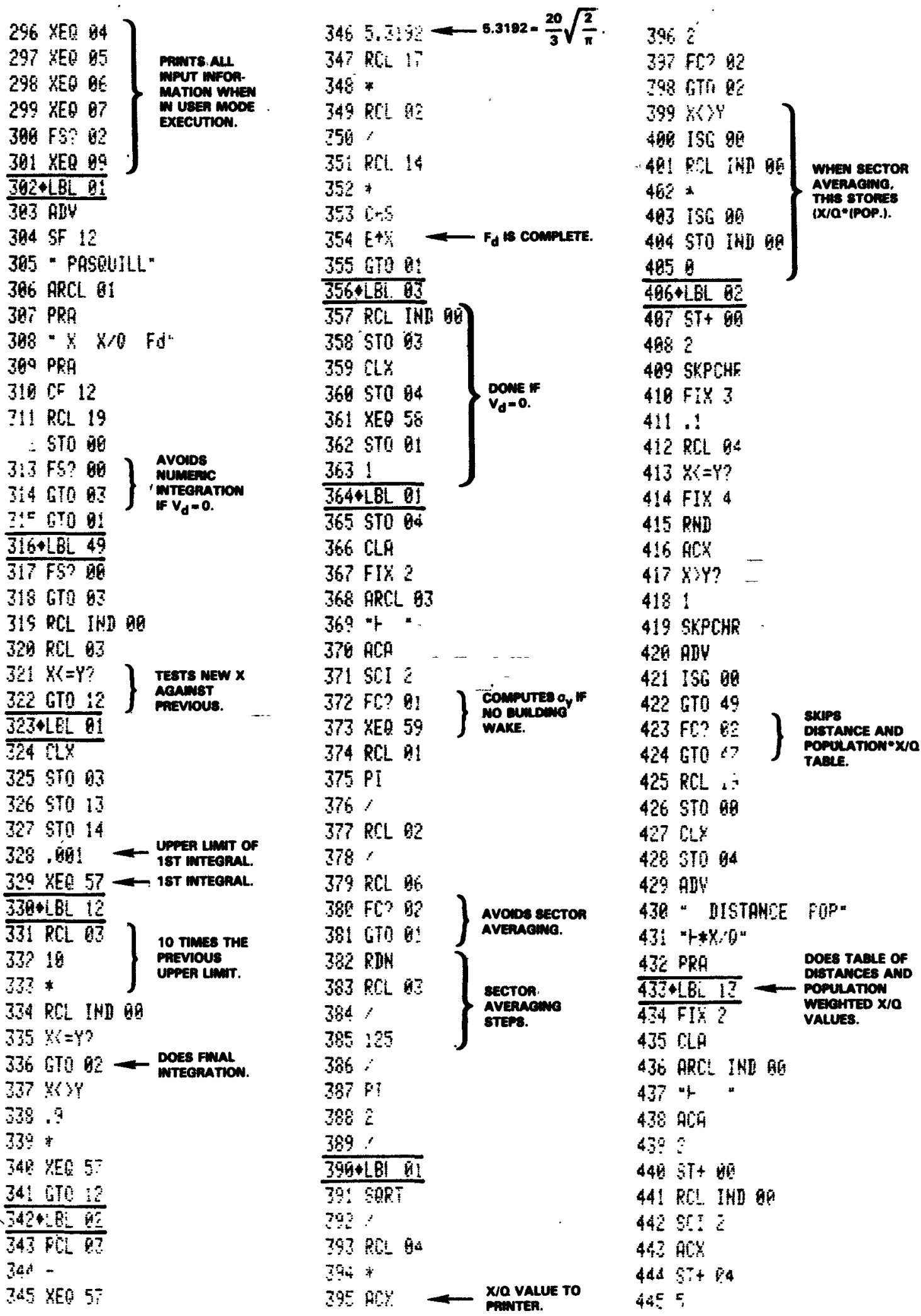




\section{PROGRAM LISTING}

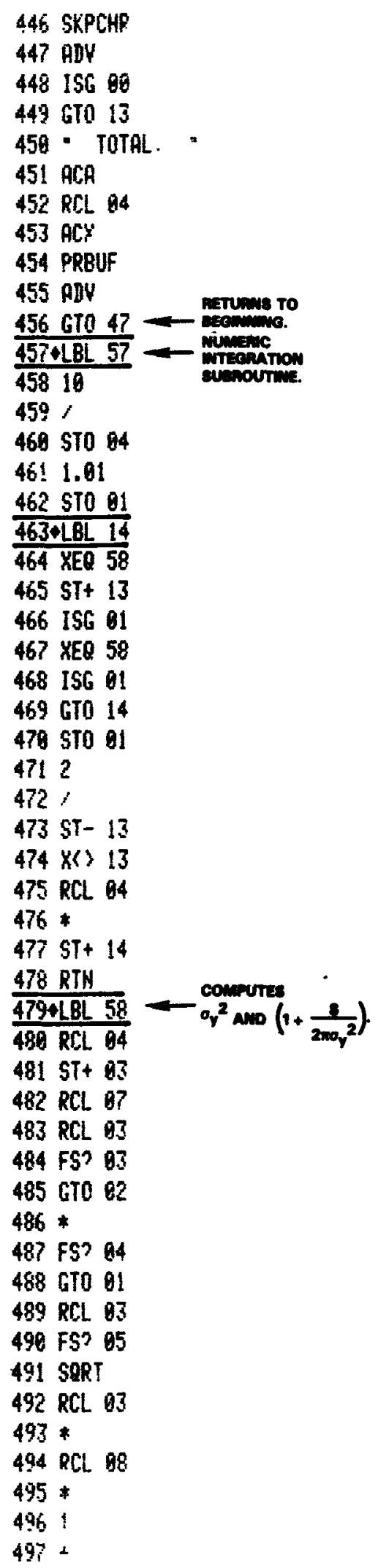

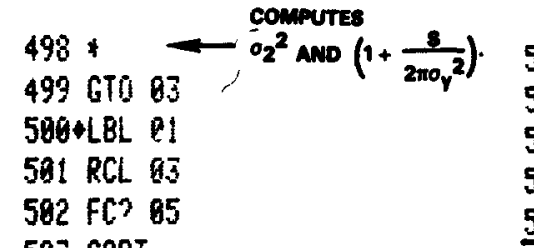

583 SQRT

594 RCL 88

$505 *$

5061

$567+$

598 FS' 95

5B9 SQRT

$510 /$

$511 \quad 670 \quad 83$

$512+L B L B 2$

$513 \mathrm{RCL} 88$

$514 *$

$515 L H_{1}+X$

516 *

$517 \cdot L B L 0_{3}$

$5182 \mathrm{E3}$

519 X>Y?

$526 X>Y Y$

$521 \times 42$

522 STO 95

$523 \mathrm{FC}$ ? 91

524 GTO 03 )

$525 \mathrm{RCL} 12$

$526 \times Y Y$

$527 ;$

5281

$529+$

530 STO 11

531 XEO 59

5329

$533 \mathrm{RCL} 10$

$534 \mathrm{RCL} 11$

$535 *$

$536 \mathrm{x}<=4$ ?

537 GTO 62

$538 \mathrm{RCL} 11$

$539 \mathrm{RCL}$ iी

$546 K=Y$ T

$54 ! 6 T 001$

542 RAD

$543 X Y Y Y$

544+LBL 0 !

5453
546 YiY?

547 GTO 81

548 STO 10

549 STn 11

550 GTO Q2

$551+$ LBL OI

$552 X+2$

$553 X\langle Y$

554 '

$555 \mathrm{FI}^{2} 43$

55611

$557 \mathrm{FS}^{\circ} 43$

55818

559 หर $>Y$

560 STO IND Y

$561+L B L$ OC

562 DES

$563 \mathrm{RCL} 11$

564 ST* 85

$565 \mathrm{RCL} 10$

566 ST* 86

567+LBL BS

568 RCL 18

$569 \times+2$

$570 \mathrm{BCL} 85$

$571 \%$

$572 \mathrm{CHS}$

$573 \mathrm{EFX}$

$574 \mathrm{RCl} 95$

575 ,

576 SOR!

$577 \mathrm{ST}+: 3$

578 RTN

5790LBL 59

$580 \mathrm{RCL} 12$

581 RCL 83

$582 \mathrm{RCL}$.

$583 *$

584 Y 4 t

$585 \mathrm{RCL} 63$

586 SORT — WTEORANO.

$58 i^{2}$

588 !

$589 \div$

$590+$

$59 !$ ।

$592510 \quad 66$

59.3 .

5941

$595+$

59657010

597 EMD 